\title{
Vortical structure in the wake of a transverse jet
}

\author{
By T. F. FRIC† AND A. ROSHKO \\ Graduate Aeronautical Laboratories, California Institute of Technology, \\ Pasadena, CA 91125, USA
}

(Received 19 August 1993 and in revised form 10 June 1994)

\begin{abstract}
Structural features resulting from the interaction of a turbulent jet issuing transversely into a uniform stream are described with the help of flow visualization and hot-wire anemometry. Jet-to-crossflow velocity ratios from 2 to 10 were investigated at crossflow Reynolds numbers from 3800 to 11400 . In particular, the origin and formation of the vortices in the wake are described and shown to be fundamentally different from the well-known phenomenon of vortex shedding from solid bluff bodies. The flow around a transwerse jet does not separate from the jet and does not shed vorticity into the wake. Instead, the wake vortices have their origins in the laminar boundary layer of the wall from which the jet issues. It is argued that the closed flow around the jet imposes an adverse pressure gradient on the wall, on the downstream lateral sides of the jet, provoking 'separation events' in the wall boundary layer on each side. These result in eruptions of boundary-layer fluid and formation of wake vortices that are convected downstream. The measured wake Strouhal frequencies, which depend on the jet-crossflow velocity ratio, match the measured frequencies of the separation events. The wake structure is most orderly and the corresponding wake Strouhal number $(0.13)$ is most sharply defined for velocity ratios near the value 4 . Measured wake profiles show deficits of both momentum and total pressure.
\end{abstract}

\section{Introduction}

The presence of a wake behind a jet in a crossflow, i.e. a 'transverse jet', has been observed in a number of previous investigations. An assumption common to many of these studies is that there is a strong similarity between the near wake of the jet and that of a circular cylinder. This view has been encouraged by observations of periodic fluctuations in the wake, reminiscent of those associated with vortices which are shed into the wake of a bluff body, and by some flow visualizations. In contrast to that idea, the investigation reported here leads to the at first surprising conclusion that there is no analogous shedding of vorticity from the jet-crossflow interaction. Instead, it is the boundary layer on the wall from which the jet issues that is the main source of vorticity for the vortical structures in the wake of the jet. This conclusion was reached from the evidence of spectral and velocity measurements and, particularly, of flow visualization, which was used to characterize the wake of the transverse jet generally and to specifically address the question of the formation of the wake structures in this complex three-dimensional flow.

Four types of coherent structure can be discerned in the near field of the jet, where the three-dimensional interaction between the jet and crossflow is most intense (Fric

$\dagger$ Present address: GE Corporate Research and Development, PO Box 8, Schenectady, NY 12301, USA. 


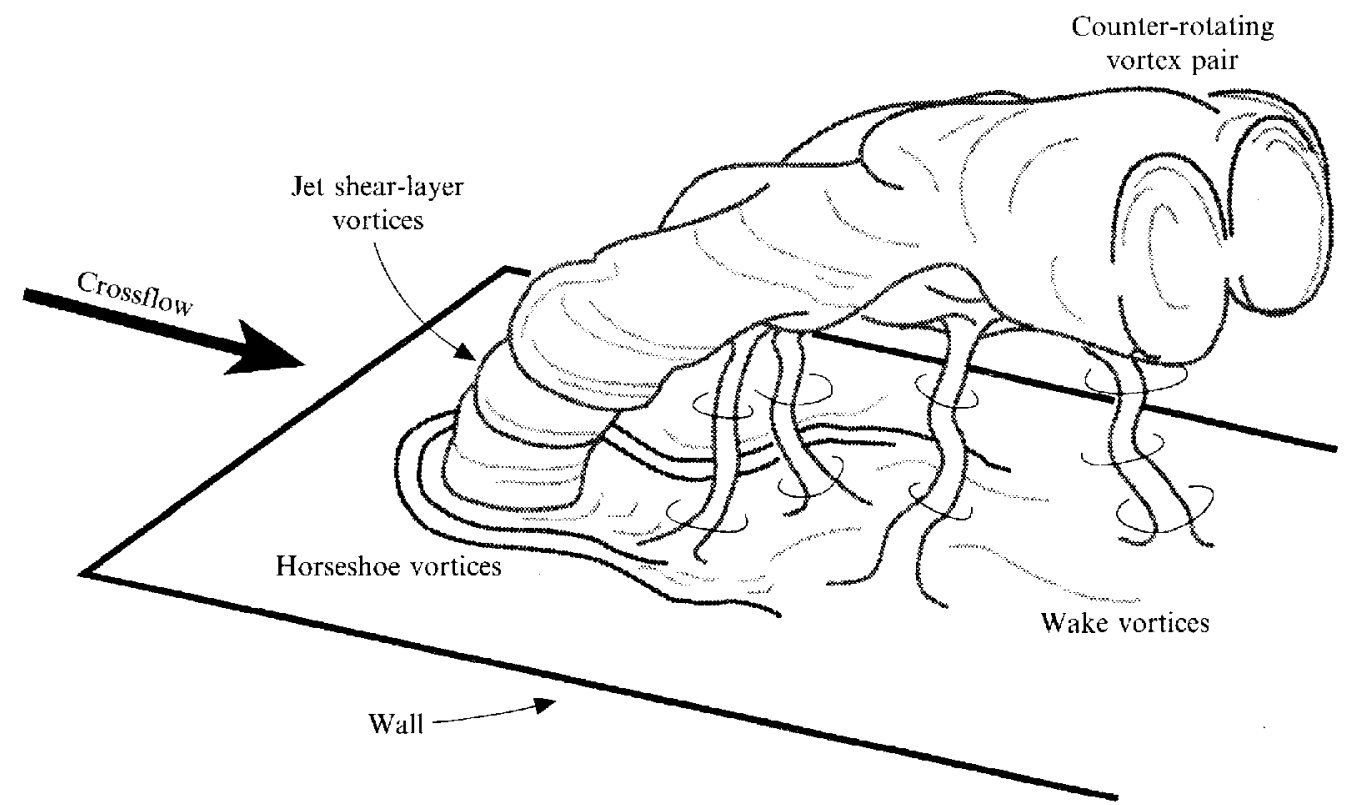

FIGURE 1. Cartoon depicting four types of vortical structure associated with the transverse-jet near field: jet shear-layer vortices at the perimeter of the bending jet, the developing counter-rotating vortex pair, horseshoe vortices on the wall, and wake vortices extending from the wall to the jet.

1990; Fric \& Roshko 1988, 1991). We begin by presenting a brief review of these structures before addressing the wake in particular. Illustrated by the cartoon in figure 1, they are the following: (i) the jet shear-layer vortices; (ii) the system of horseshoe vortices; (iii) the incipient, counter-rotating vortex pair; and (iv) the wake vortices. Two of these, the shear-layer vortices and the wake vortices, are intrinsically unsteady; the other two, i.e. the horseshoe vortices and the vortex pair, have mean-flow definition although they may also have unsteady components.

The two photographs in figure 2 clearly show the jet shear-layer vortices, which dominate the initial portion of the jet and which are a result of the Kelvin-Helmholtz instability of the annular shear layer that separates from the edge of the jet orifice. Figure 2(a) shows a visualization of a transverse jet as it is typically viewed from the side, using smoke seeding of all the entering jet fluid to visualize its trajectory. In figure $2(b)$, smoke streaklines in the centreplane of the crossflow are entrained into and visualize the leading edge of the deflected jet. In this photograph some smoke is faintly seen coming out of the centreplane from axial flow along the cores of the first few distorted vortex rings. These structures are of the same type as the vortex ring structures of free jets (Freymuth 1966; Becker \& Massaro 1968; Gutmark \& Ho 1983).

Two views of horseshoe vortices wrapping around the base of a jet issuing from a wall into a crossflow are shown in figure 3. In figure 3(a), a plan view of two horseshoe vortices is shown: they are distinct structures until just downstream of the jet orifice, where the turbulence of the jet's wake is evident. Figure $3(b)$ shows a cross-sectional view of two horseshoe vortices for the same flow, which shows the flow pattern produced by the interaction of the horseshoe vortices upstream of the jet and the Kelvin-Helmholtz vortices in the leading-edge shear layer of the emerging jet. For comparison, figure $3(c)$ shows a horseshoe-vortex system for the flow around the base of a wall-mounted circular cylinder. For both the jet and the cylinder, the approaching 
(a)

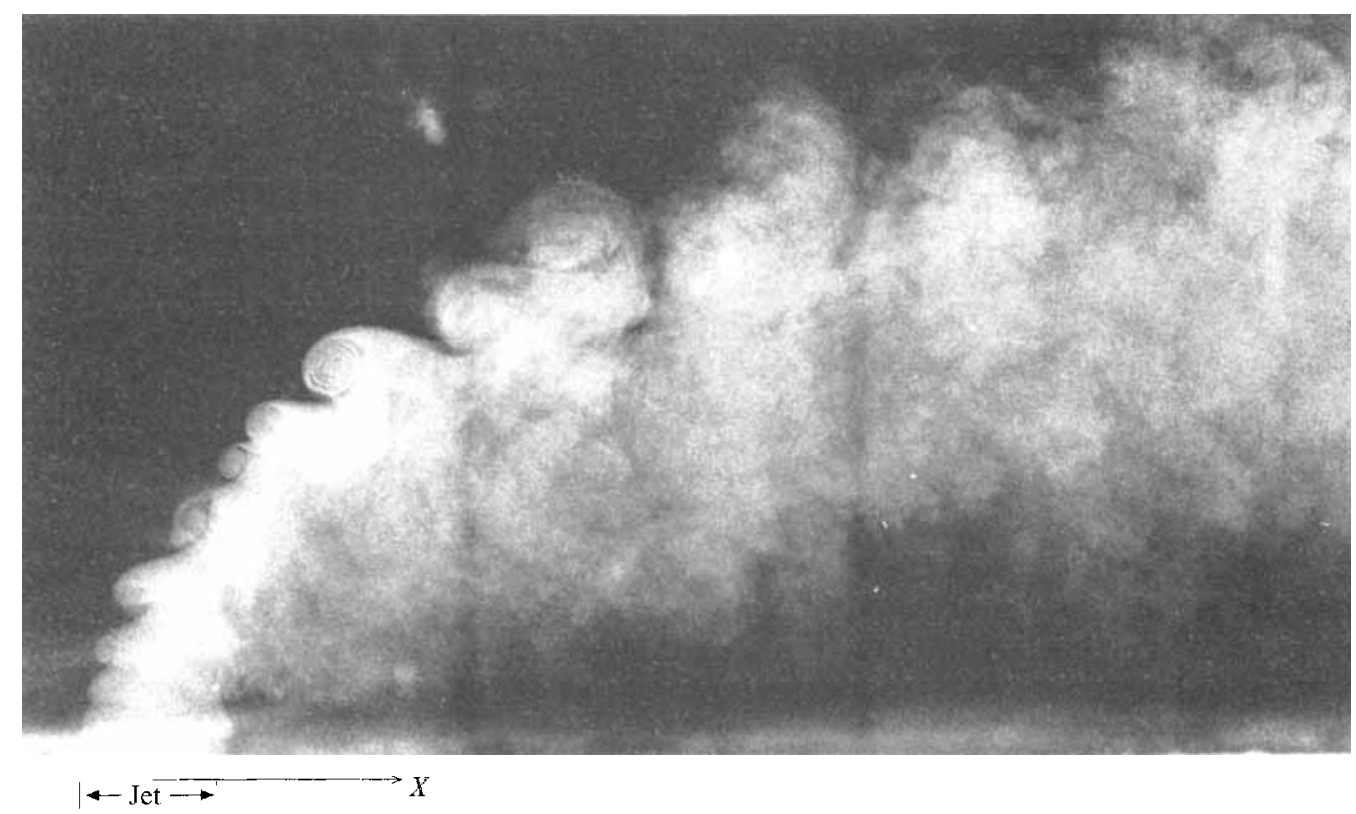

(b)

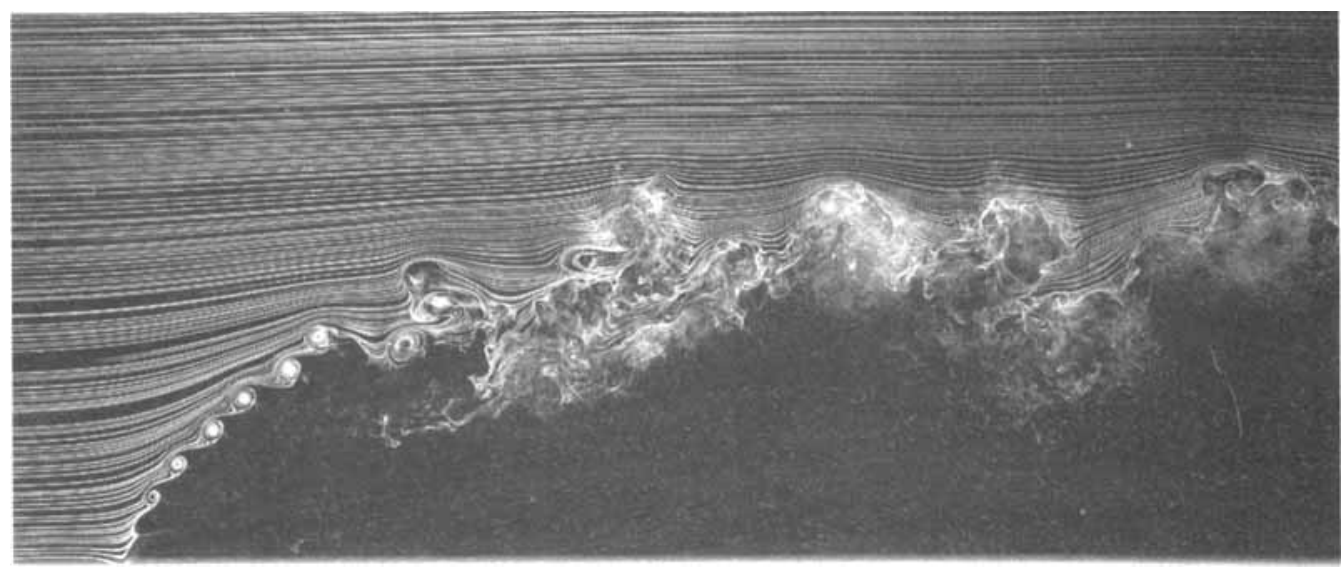

$1 \leftrightarrow \mathrm{Jct} \rightarrow \mathrm{T} \longrightarrow \mathrm{X}$

FIGURE 2. Transverse-jet shear-layer structure visualized by $(a)$ a smoke-filled jet and by $(b)$ smoke streaklines entrained into the leading edge of the jet. $V_{R}=2, R e_{c f}=3800$.

wall boundary layer encounters an adverse pressure gradient ahead of the obstacle (the jet or cylinder) and separates to form horseshoe vortices.

The third type of structure we list is the vortex pair, which occurs as a result of the impulse of the jet on the crossflow (Scorer 1958, pp. 193--195; Broadwell \& Brcidenthal 1984). It begins to take form in the near field, as indicated in figure 1 , and becomes dominant in the far field, where it is synonymous with the 'jet'. A partial list of the numerous experimental studies of the vortex-pair structure of the jet includes the 
(a)

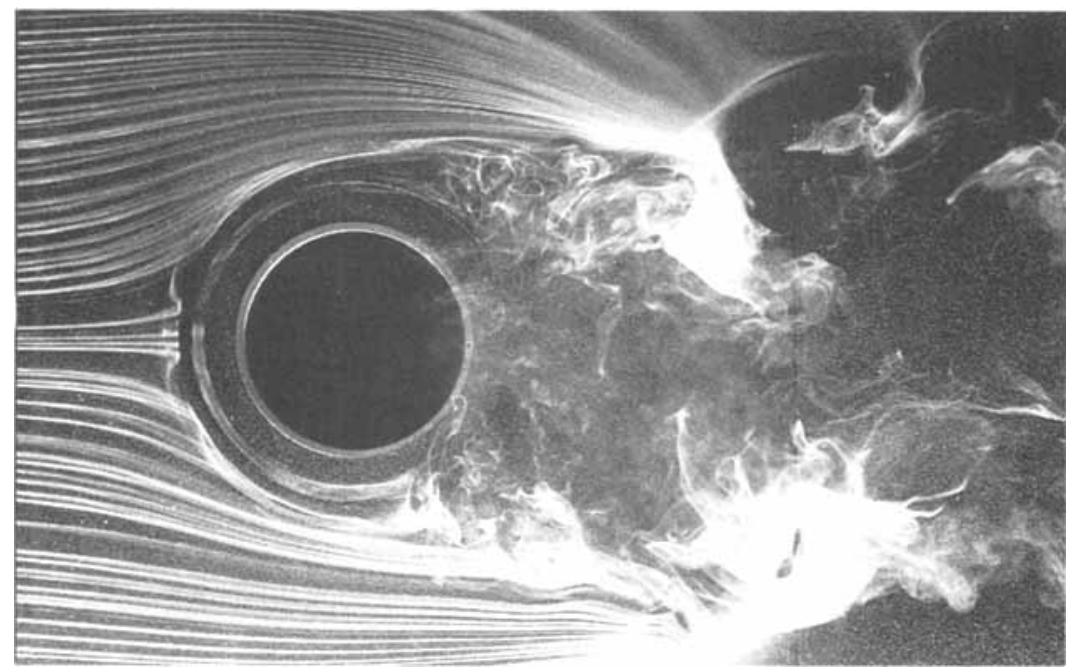

(b)

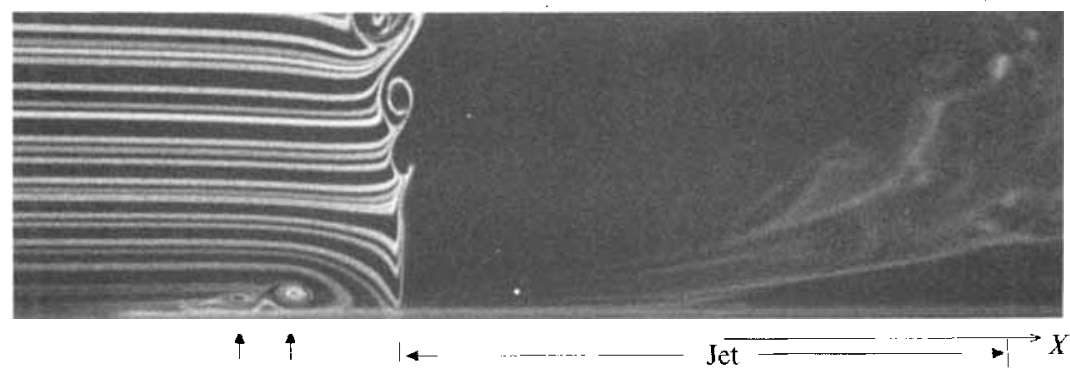

(c)

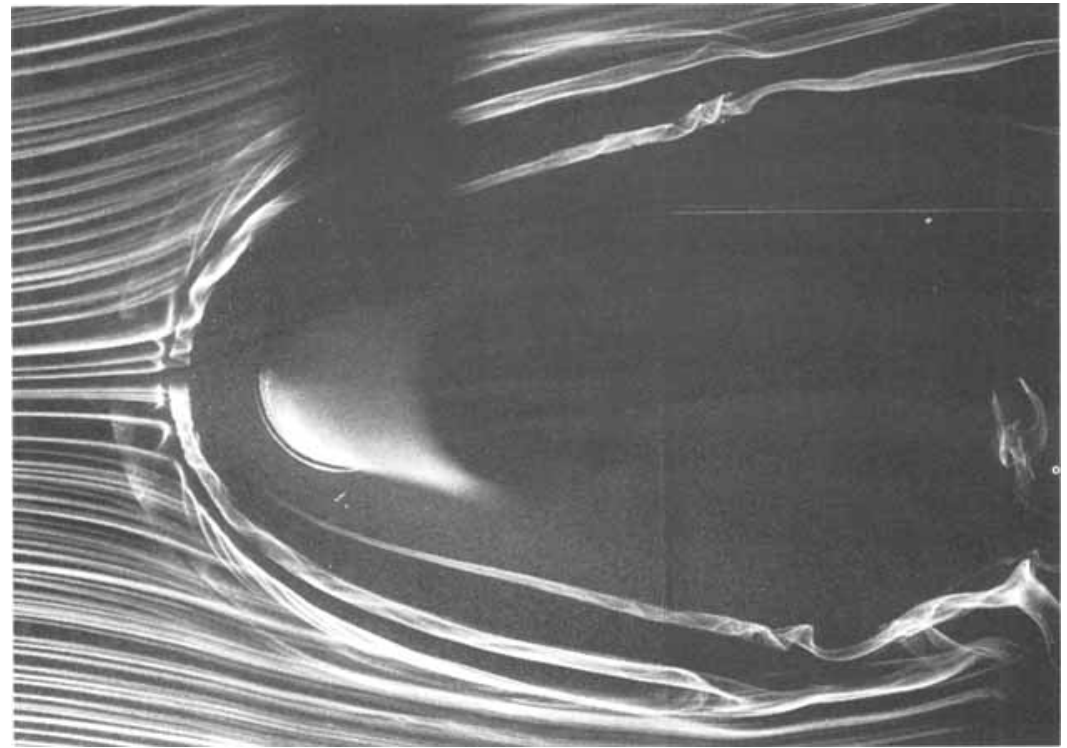

FIGURE 3. Horseshoe-vortex system at the wall. (a) Plan view at the base of a transverse jet with jetto-crossflow velocity ratio $V_{R}=10,(b)$ centreplane section showing two horseshoe vortices (indicated by arrows) upstream of a $V_{R}=10$ jet and their interaction with shear-layer vortices of the emerging jet, and (c) plan view at the base of a wall-mounted circular cylinder. Crossflow Reynolds number equals 3800 in each case. 
following: Keffer \& Baines (1963), Pratte \& Baines (1967), Kamotani \& Greber (1972), and Fearn \& Weston (1974).

Finally, also sketched in figure 1 are the wake vortices, which are the main subject of this paper. The wake vortices are perhaps the most intriguing structures in the near field. They have some characteristics which are similar to wake vortices of solid cylinders, but differences between the jet (a fluid) and the cylinder (a solid) as obstacles to the crossflow suggest significant differences in how the wake vortices should form. Furthermore, if the 'wake' is defined as the region downstream of the jet, between the jet and the wall, it can immediately be seen that this is fundamentally different from a bluff-body wake. Here, the deflecting jet follows the wake downstream, continually enlarging its span. Each wake vortex, as a structure, has a termination on the wall, just as behind a bluff body, but the other end terminates on the jet itself. This coincidental development of the jet and wake presents a further conceptual difficulty for comparison with 'shedding' from a two-dimensional cylinder.

It should be emphasized that the counter-rotating vortex pair and the wake vortices are quite distinct, different structures, with differen 1 generic origins. The vortex pair is essentially a manifestation of the mean flow field induced by the impulse of the initial (normal) jet, which can be considered simply as providing a transverse force, cf. a lifting wing. This point is elucidated by Broadwell \& Breidenthal (1984). The trajectory of the jet is really composed of two elements - the motion carrying it further from the wall in the transverse direction, resulting from the initial impulse or jet direction, and the motion carrying the trajectory in the crossflow direction. The latter is a result of the entrainment of crossflow fluid by the jet (Coehlo \& Hunt 1989). In the near field the jet does have a displacement effect on the crossflow, and through that an effect on the wall boundary layer, inducing both the horseshoe vortices and wake vortices. The connection between the wake vortices and the wall boundary layer is an important aspect of our results that will be developed in later sections.

In many transverse-jet experimental and numerical studies the role of the wake has generally not been addressed. Even though the wake has been thought to contain vortical structure similar to that in wakes of cylinders, much of the past emphasis has been on the counter-rotating vortex pair, and relatively few investigators have measured or visualized structure in the wake region. Some of the exceptions are McAllister (1968) and Reilly (1968), who extracted wake Strouhal frequencies from flow visualization, and McMahon, Hester \& Palfrey (1971) and Moussa, Trischka \& Eskanazi (1977), who detected characteristic wake frequencies from hot-wire measurements. McMahon et al. also visualized structure in the wake of the jet by placing a mesh of tufts across the wake. Kuzo \& Roshko (1984), using dye injected into the wake, visualized the wake vortices and found that they exist at least a hundred jet diameters downstream of the orifice. In fact, those far-field wake experiments in part motivated the present investigation of the near-field wake. More recently, Wu, Vakili \& Yu (1988) visualized coherent wake vortices behind asymmetric jets in crossflow, referring to them as 'spin-off' vortices. Those vortices were visualized by placing the dye port in the wake just downstream of the jet. Wu et al. (1988) did not report wake (or 'spin-off') vortices for symmetric jets.

We reiterate that the usual point of view in observations of transverse jets is that the formation of their wake vortices must be analogous to the shedding of vortices from solid cylinders. But the fact that the jet boundary is not a solid surface presents a difficulty for vortex shedding and suggests that this may lead to a fundamental difference between the two kind of wakes. In earlier studies in which the issue was first addressed (Fric 1990; Fric \& Roshko 1991), we suggested that transverse-jet wake 


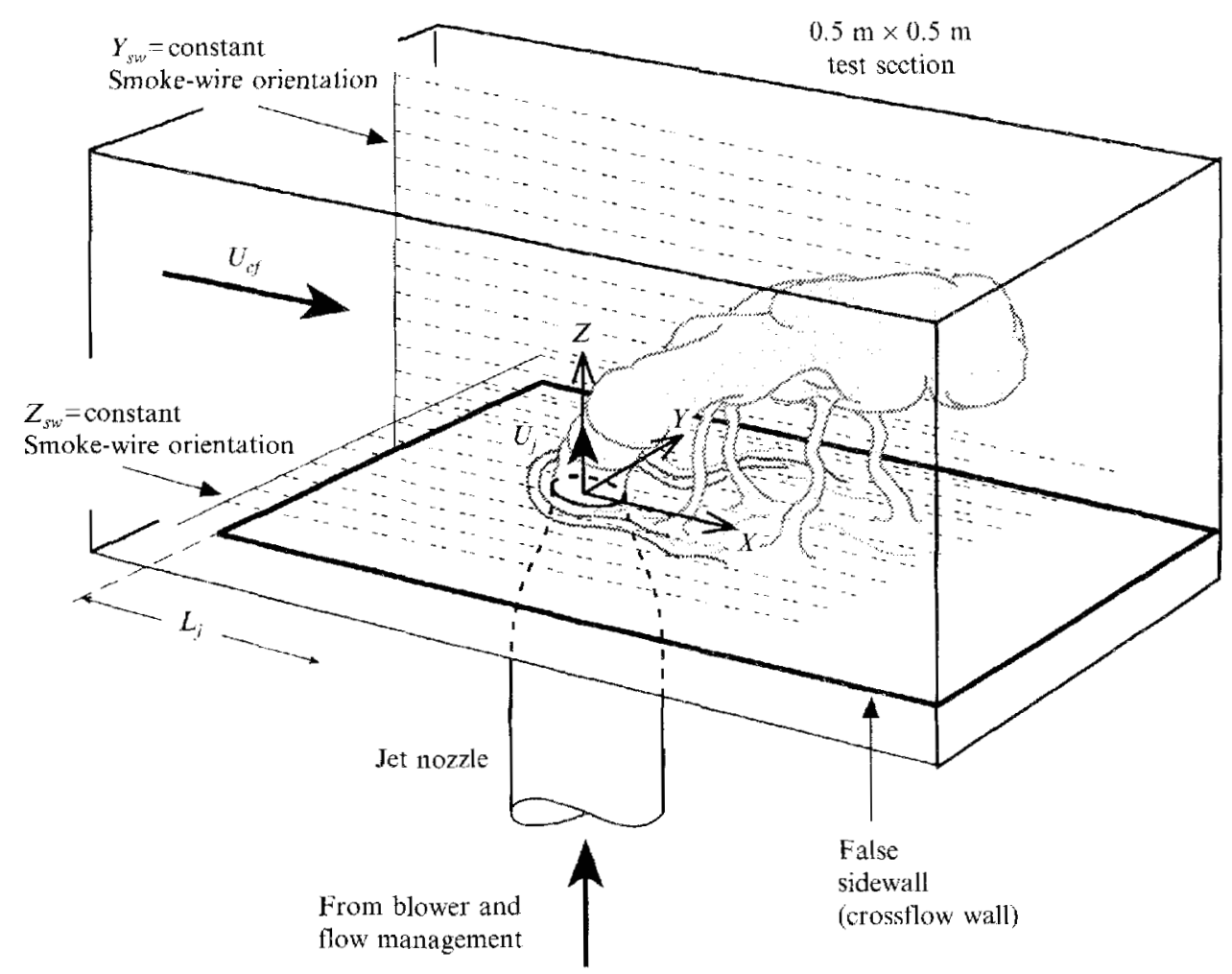

FIgure 4. Details of the experimental set-up with the jet injected at $90^{\circ}$ to the crossflow.

vorticity originates from the boundary layer on the wall from which the jet issues. To obtain better understanding of the differences between cylinder and jet wakes and of the formation of transverse-jet wakc vortices, we werc led to the experimental studies described here. Throughout, the emphasis of this paper is on describing the near-field jet wake and presenting a mechanism of wake formation which is consistent with our observations and with vorticity generation theory. Establishing the source of vorticity for the wake structures was considered essential to understanding them.

The experimental apparatus and techniques used are briefly described in $\$ 2$. Some of the wake results from smoke-wire flow visualization are shown in $\S 3$. Wake Strouhal frequency measurements, and velocity and total pressure profiles in the transverse jet wake are presented in $\$ 4$ and 5 , respectively. The issue of sources of vorticity is discussed in $\$ 6.1$, while its significance to the present problem is addressed in $\$ 6.2$. In $\$ \$ 6.3$ and 6.4 a series of specific flow visualizations and measurements are then used to show that the source of the wake vorticity is the boundary layer on the wall from which the jet issues. Comparisons are made between the transverse jet wake and circular cylinder wake. Having established the source of vorticity, a wake formation mechanism is proposed and discussed in $\$ 6.5$. Other results not discussed here are available in Fric (1990).

\section{Experimental set-up}

The experiments were done in an open-return low-speed wind tunnel of cross-section $0.5 \mathrm{~m}$ by $0.5 \mathrm{~m}$. Nominal mean crossflow velocities $U_{c /}$ ranged from 1.5 to $4.5 \mathrm{~m} \mathrm{~s}^{-1}$; 
flow uniformity across the span of the test section upstream of the jet installation was better than $2 \%$. Turbulence intensity levels were measured at $0.25 \%, 0.2 \%$, and $0.2 \%$ for $U_{i f}=1.5,3$ and $4.5 \mathrm{~m} \mathrm{~s}^{-1}$, respectively. The wind tunnel test section with the jet installation and other elements of the experimental set-up are shown in figure 4 . Some of the experimental issues are discussed below. More details are given in Fric (1990).

Since the flow at the surface from which the jet issues was found to be important during the early stages of these experiments, a false wall into which the jet nozzle exit was flush-mounted was installed in the test section, as shown in figure 4 . This provided better control of the boundary layer on the wall through which the jet was injected. In order to avoid a separation bubble at the contoured leading edge of the false wall, the pressure drop through the crossflow portion of the test section had to be nearly matched to that through the gap between the false wall and tunnel wall. This was accomplished by means of control screens of appropriate solidity at the downstream end of the crossflow portion of the test section. (Note that all references to 'downstream' and 'upstrcam' in this paper denote the nominal crossflow direction.) In the remainder of this paper, the false wall is called the 'crossflow wall' and the boundary layer on it the 'crossflow boundary layer'. The internal boundary layer on the wall of the jet nozzle is called the 'jet boundary layer'.

Table 1 shows measured nominal crossflow boundary-layer displacement thicknesses $\delta_{c f}$ at $X / D_{j}=0$ for each of three nominal crossflow velocities and for two crossflowwall upstream lengths $L_{j} / D_{j} . D_{j}$ is the jet diameter. (Nominal conditions are those where the effects of the jet on the crossflow and vice versa are not present.) $L_{j}$ represents the distance from the leading edge of the crossflow wall to the centre of the jet orifice; $L_{j} / D_{j}$ was either 5 or 10 in these experiments. In addition, table 1 shows turbulence intensity levels $u_{r m s}^{\prime} / U_{c f}$ in the boundary layer at $Z$ where the mean velocity $U=U_{c f} / 2$. It shows that the undisturbed or nominal crossflow boundary layer is laminar in all cases. The crossflow Reynolds number $R e_{c f}$ is defined as $U_{c f} D_{j} / \nu$, where $\nu$ is the kinematic viscosity of air.

The incompressible jet which issues into the crossflow was supplied by a $3.8 \mathrm{~cm}$ exitdiameter nozzle. A matched cubic contour, with its point of inflection at $60 \%$ from the nozzle inlet, was selected for the nozzle contraction, and the nozzle area contraction ratio was nine. The nozzle was designed to prevent boundary-layer separation at its inlet, to prevent the formation of Görtler vortices on its concave portion, and to produce a nearly top-hat velocity profile at its exit. The jet was powered by a radial vane centrifugal blower. Standard techniques were used in the design of the flow management section (screens and honeycomb) located between the blower and nozzle. Jet velocity $U_{j}$ was continuously variable between 3 and $45 \mathrm{~m} \mathrm{~s}^{-1}$. Table 2 shows the nominal jet-exit boundary-layer displacement thickness $\delta_{j}$ and potential-core turbulence intensity levels $w_{r m s}^{\prime} / U_{j}$ for the relevant range of jet Reynolds numbers $R e_{j}$, which are defined as $U_{j} D_{j} / \nu$.

An important parameter defining the transverse jet is the jet to crossflow velocity ratio $V_{R}$. In these experiments, $2 \leqslant V_{R} \leqslant 10$. For $V_{R}$ in this range, and $D_{j}=3.8 \mathrm{~cm}$, the trajectory of the jet is such that any interference effects of the wall opposite to the crossflow wall, 12 jet diameters away, should not affect the present results and conclusions about the mechanism of wake vortex formation.

The smoke-wire flow visualization technique, which places closely spaced streaklines into the flow, was used extensively in this study. This technique is described by Corke et al. (1977). In each of the instantaneous photographs included in this paper the jet is issuing toward the viewer or, in the conventional side view, from the bottom of the photograph. The crossflow is always from left to right, and the smoke wire is typically 


\begin{tabular}{|c|c|c|c|c|}
\hline $\begin{array}{c}U_{e f} \\
\left(\mathrm{~m} \mathrm{~s}^{-1}\right)\end{array}$ & $\boldsymbol{R} e_{n f}$ & $L_{j} / D_{j}$ & $\delta_{c f f} / D_{j}$ & $\begin{array}{c}u_{r m s}^{\prime} / U_{c f} \\
\text { (where } U / U_{e f}=0.5 \text { ) }\end{array}$ \\
\hline 1.5 & 3800 & 5 & 0.056 & 0.0046 \\
\hline 1.5 & 3800 & 10 & 0.087 & 0.0046 \\
\hline 3.0 & 7600 & 5 & 0.039 & 0.0044 \\
\hline 3.0 & 7600 & 10 & 0.055 & 0.0041 \\
\hline 4.5 & 11400 & 5 & 0.029 & 0.0073 \\
\hline 4.5 & 11400 & 10 & - & 0.0040 \\
\hline
\end{tabular}

TABLE 1. Displacement thicknesses and turbulence intensity levels of the nominal crossflow boundary layer at $X / D_{j}=0$.

\begin{tabular}{cccc}
$\begin{array}{c}U_{j} \\
\left(\mathrm{~m} \mathrm{~s}^{-1}\right)\end{array}$ & \multicolumn{1}{c}{$R_{j}$} & $\delta_{j} / D_{j}$ & (at $X=Y=Z=0$ ) \\
3 & 7600 & 0.045 & 0.012 \\
6 & 15200 & 0.039 & 0.012 \\
9 & 22900 & 0.037 & 0.012 \\
12 & 30500 & 0.035 & 0.012 \\
15 & 38100 & 0.033 & 0.010 \\
18 & 45700 & 0.032 & 0.0098 \\
24 & 61000 & 0.031 & 0.0079 \\
27 & 68600 & 0.031 & 0.0072 \\
30 & 76200 & 0.031 & 0.0067 \\
36 & 91400 & 0.030 & 0.0062 \\
45 & 114000 & 0.029 & 0.0052
\end{tabular}

TABLE 2. Displacement thicknesses of the nozzle boundary layer, and potential core turbulence intensities at the exit of the jet nozzle $\left(Z / D_{j}=0\right)$ in the absence of crossflows, i.e. at nominal conditions.

$\begin{array}{ccc}R e_{c f} & V_{R} & V_{R_{\text {actual }}} \\ 3800 & 2 & 2.26 \\ & 4 & 4.19 \\ & 6 & 6.17 \\ & 8 & 8.16 \\ 7600 & 10 & 10.5 \\ & 2 & 2.07 \\ & 4 & 3.98 \\ & 6 & 6.12 \\ & 8 & 8.30 \\ 11400 & 10 & 10.6 \\ & 2 & 2.02 \\ & 4 & 4.08 \\ & 6 & 6.23 \\ & 8 & 8.42 \\ & 10 & 10.7\end{array}$

TABLE 3. Comparison of nominal $\left(V_{R}\right)$ and actual $\left(V_{R_{\text {actual }}}\right)$ velocity ratios. 
(a)

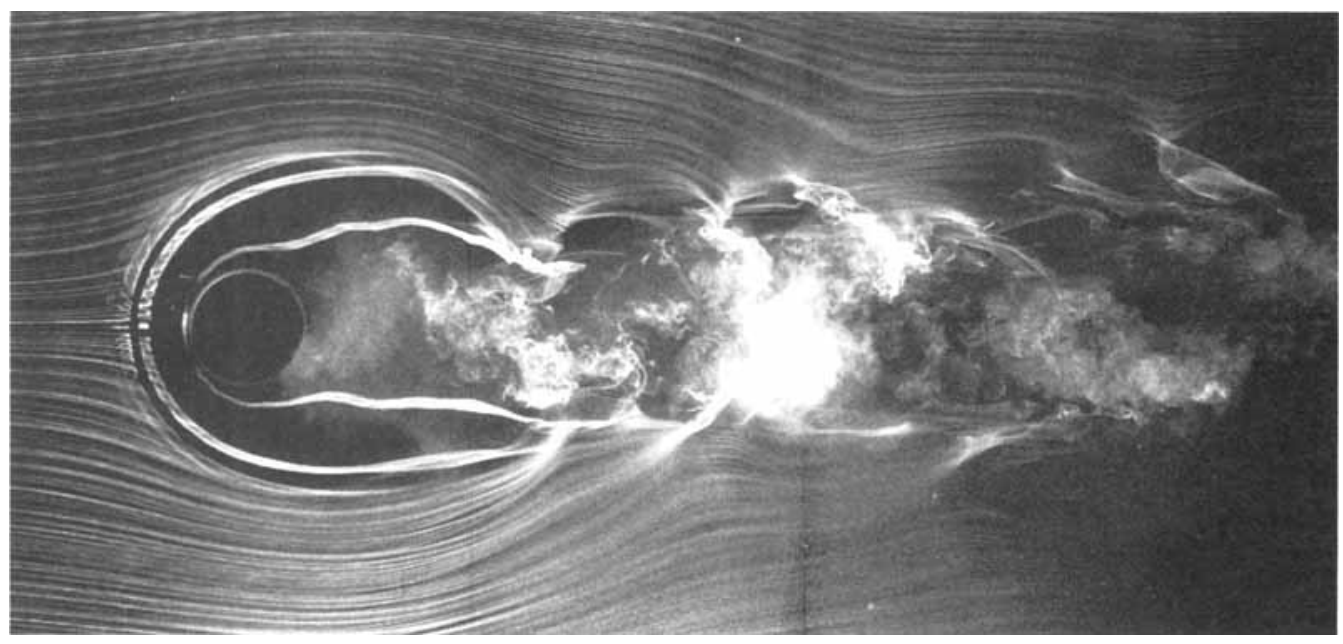

(b)

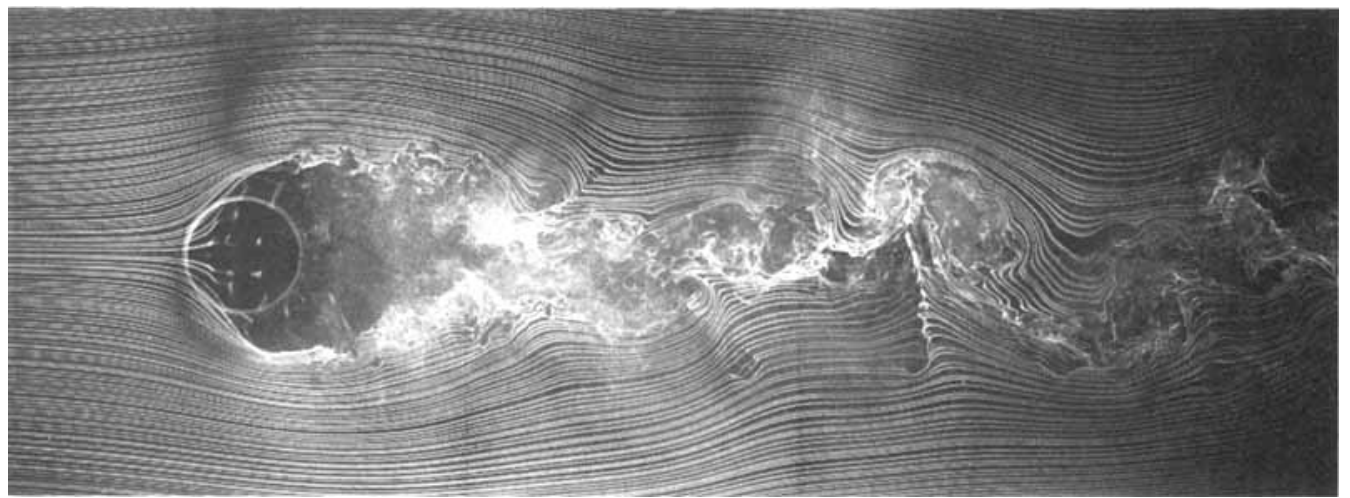

(c)

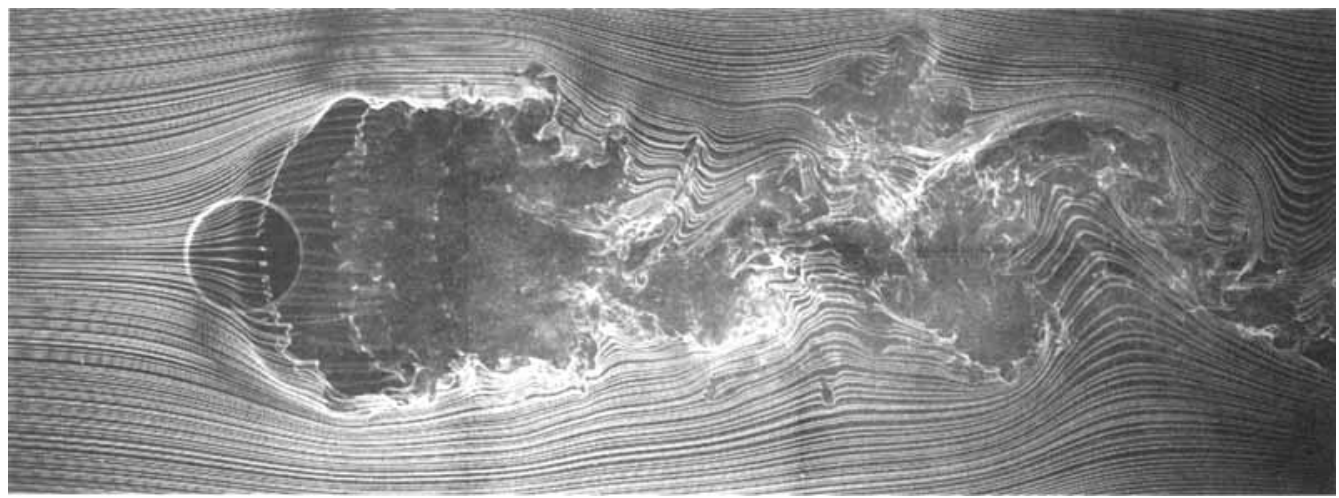

Figure 5. Cross-sectional views of the flow around jets and their wakes for velocity ratio $V_{R}=2$. $R e_{c f}=7600$. Smoke streaklines originate at different distances from the crossflow wall for each photograph: (a) $Z_{s w} / D_{j}=0+;$ (b) $Z_{s w} / D_{j}=0.5 ;$ (c) $Z_{s w} / D_{j}=1.0$ Figures 6.9 show corresponding photographs for jets at higher velocity ratios. 
(a)

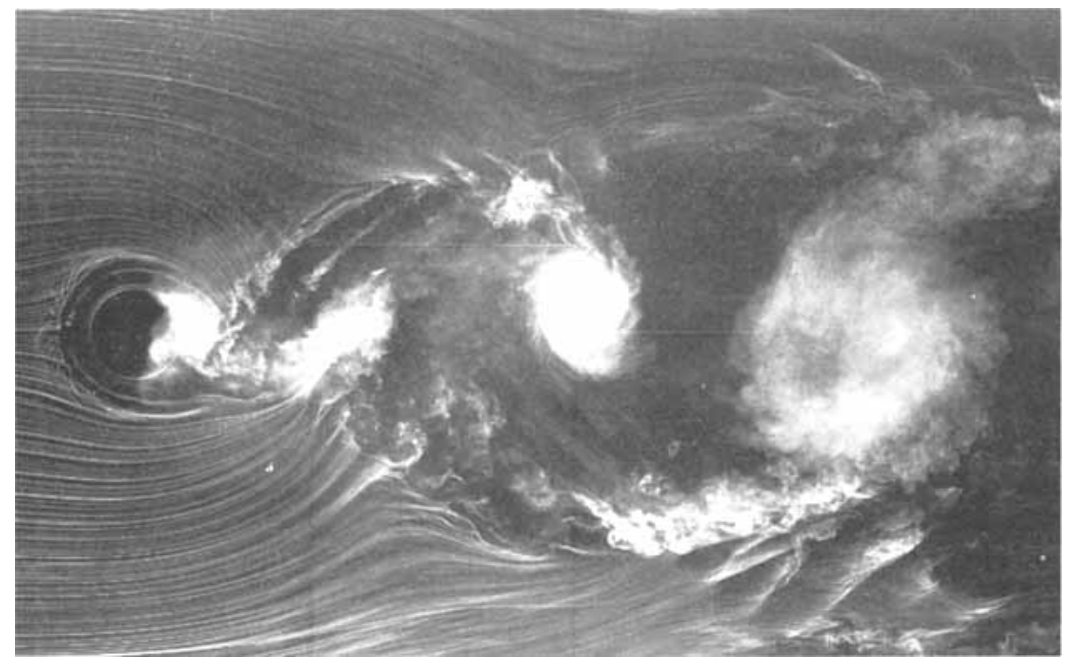

(b)

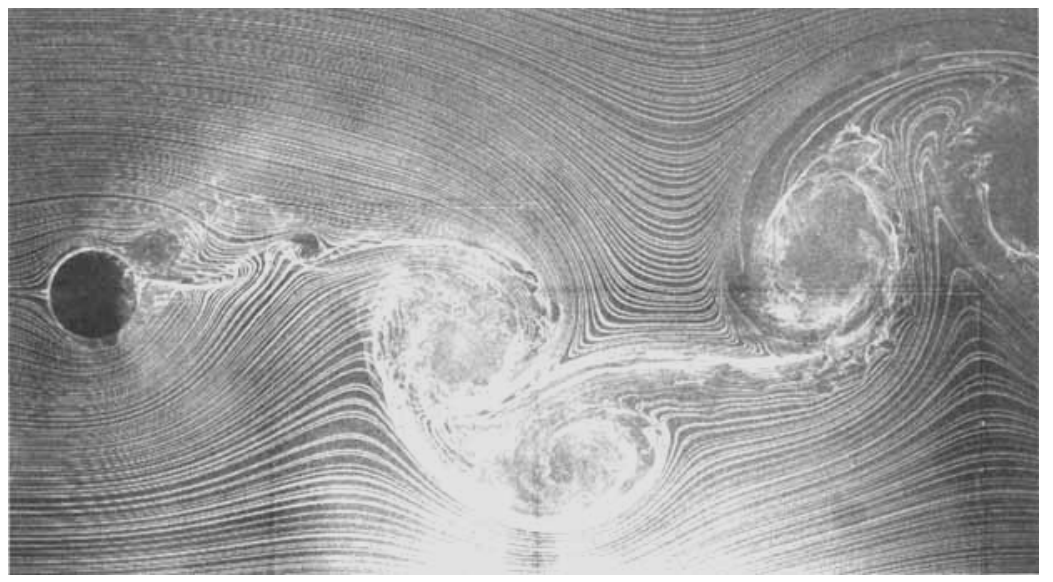

(c)

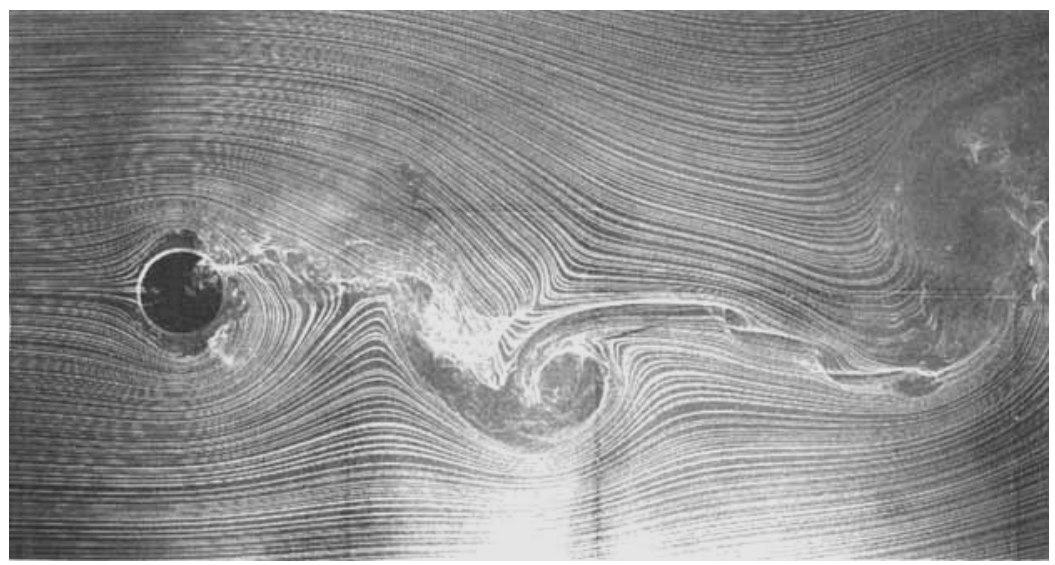

FIGURe 6. Cross-sectional views for $V_{R}=4$. $R e_{c f}=11400$. (a) $Z_{s w} / D_{j}=0+$; (b) $Z_{s w} / D_{j}=0.5$; (c) $Z_{s w} / D_{j}=1.0$. 
(a)

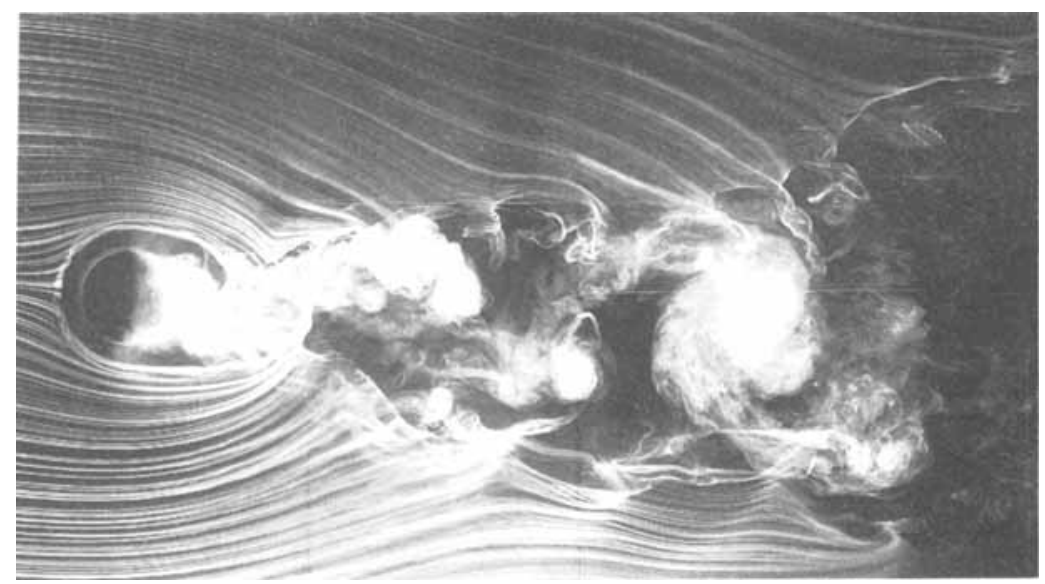

(b)

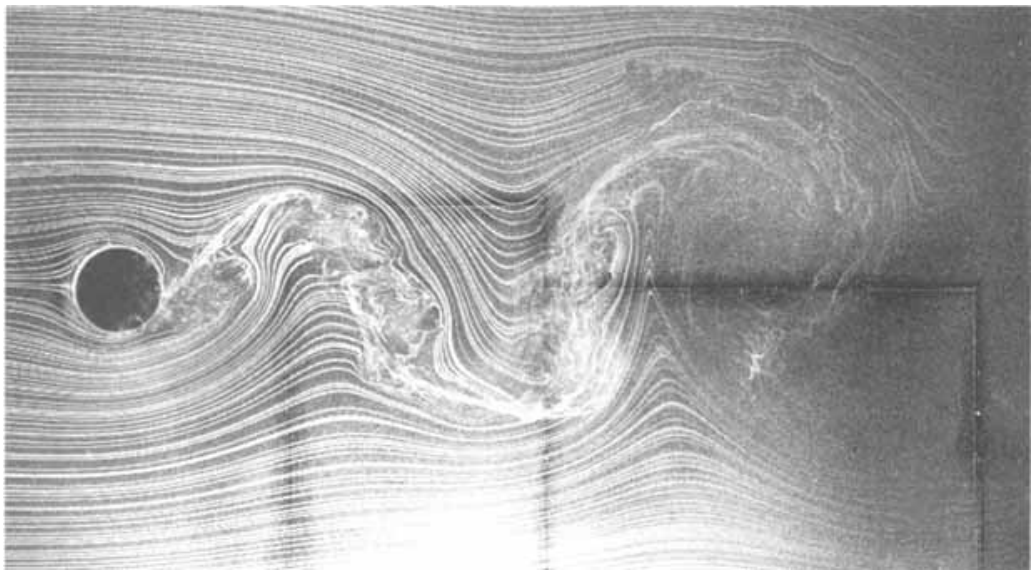

(c)

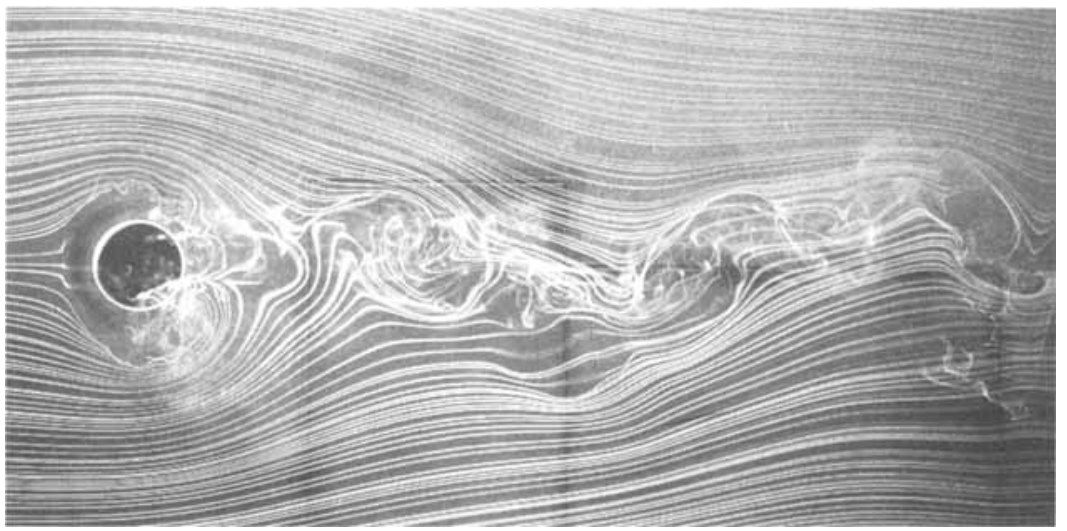

Figure 7. Cross-sectional views for $V_{R}=6 . R e_{c f}=3800 .(a) Z_{s w} / D_{j}=0+;(b) Z_{s w} / D_{j}=0.5$; (c) $Z_{\varepsilon * t} / D_{3}=2.0$. 
upstream of the viewing area. Two common smoke-wire orientations are depicted in figure 4. In some cases the smoke streaklines begin in a $Y=$ constant plane while in other cases they begin in a $Z=$ constant plane. We shall refer to the corresponding pictures as 'cross-sectional' or simply 'sectional' views, but in fact the smoke can migrate out of the plane of injection as the flow dictates and, since general lighting was used and not 'sheets' of light, this three-dimensionality is photographed. It should be noted that for the cases where the jet issues toward the viewer the camera is not necessarily coincident with the $Z$-axis. Therefore using the crossflow wall in the background of the photographs to visually gauge the $X$-position of flow features relative to the jet orifice can be slightly inaccurate. The photographs, obtained with the smoke-wire technique, show that the transverse jet wake has a rich and complex structure. When the smoke is injected directly into vorticity-carrying fluid, such as the crossflow boundary layer or jet boundary layer, it marks the progress of that vortical fluid and the formation of vortices, if that occurs. (The smoke, however, diffuses at a rate slower than that of the vorticity.) When the smoke is injected into irrotational flow, it may still reveal the formation of vortices when it is caught up in circulating flows.

TSI single hot-wire probes, traversed by a computer-controlled XYZ traversing system, were used for velocity and spectral measurements. Power spectra were obtained using an HP3582A real time spectrum analyser, and the characteristic dominant flow frequencies were obtained from the r.m.s. average of 32 individual spectra. Wake total pressures were also measured, using a United Sensor Venturi Kiel probe in conjunction with a Datametrics pressure sensor and electronic manometer.

Values of crossflow velocity $U_{c f}$ and jet velocity $U_{j}$ used here are nominal values. That is, they are the crossflow conditions with no jet flow and jet conditions with no crossflow, respectively. Turning on either the crossflow or the jet changes the velocity of the other and therefore the nominal and actual velocities are not in general the same. Table 3 lists nominal velocity ratios $V_{R}$ and the corresponding actual values, $V_{R_{a c t u a l}}$. Contributing factors to the difference between the two include pressure changes in the wind tunnel test section and blockage effects of the deflecting jet. The actual velocity ratios generally prove to be slightly greater than the nominal ones.

\section{Visualization of wake structure}

It is appropriate to begin our presentation of results with flow visualization, for it was visualization of the wake structure which gave important insight into the flow and guided us in subsequent measurements. In this section we present photographs which are examples of cross-sectional and side views of the jet-wake structure for the range of velocity ratios from 2 to 10 . For both views, comparisons are made with the wake of a solid cylinder which is attached at one end to the crossflow wall and is free at the other. Immediately important differences between jet and cylinder wakes will be evident, and it was differences such as these which led us to investigate jet-wake vorticity origins more carefully. The side views of the jet-wake structure are the first results shown which suggest that the source of vorticity for the jet wake is the crossflow boundary layer.

In discussing the flow visualizations, terms such as 'vortical structure' and 'vortex' will be used equivalently to refer to a flow feature if it is relatively well organized and appears rotational in nature. Although this is subjective and leaves the determination open to some interpretation, what can be considered a vortical structure is quite clear in most cases. 
(a)

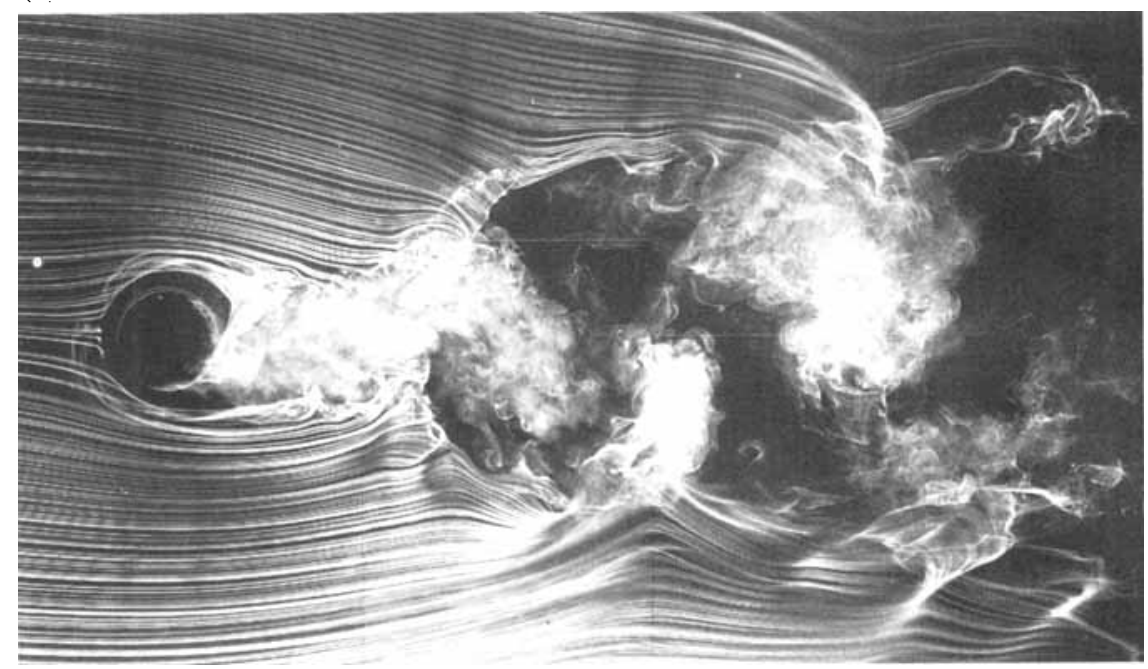

(b)

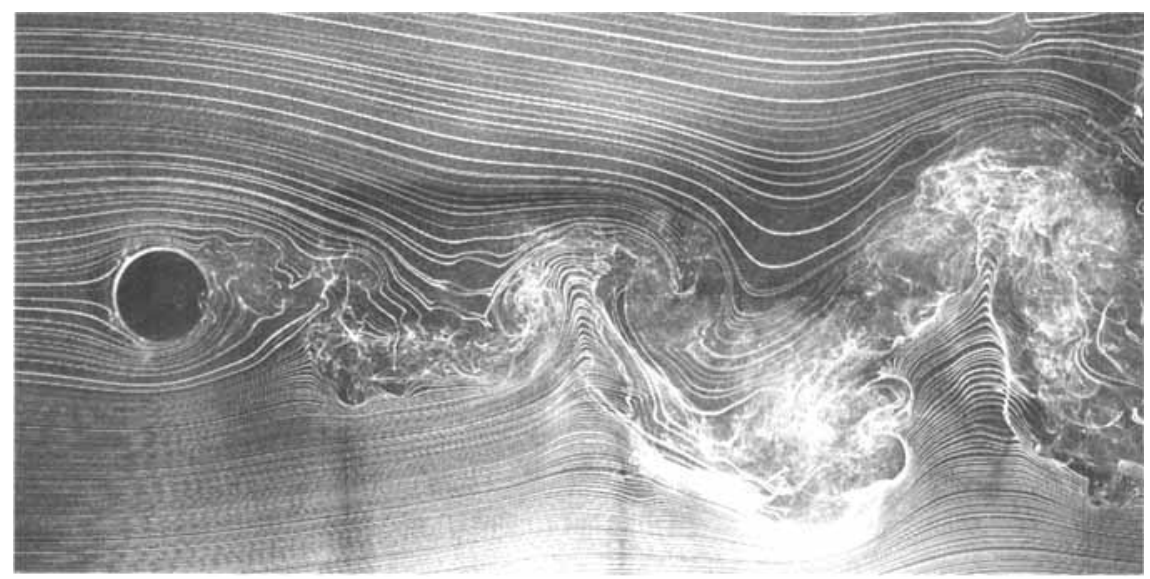

(c)

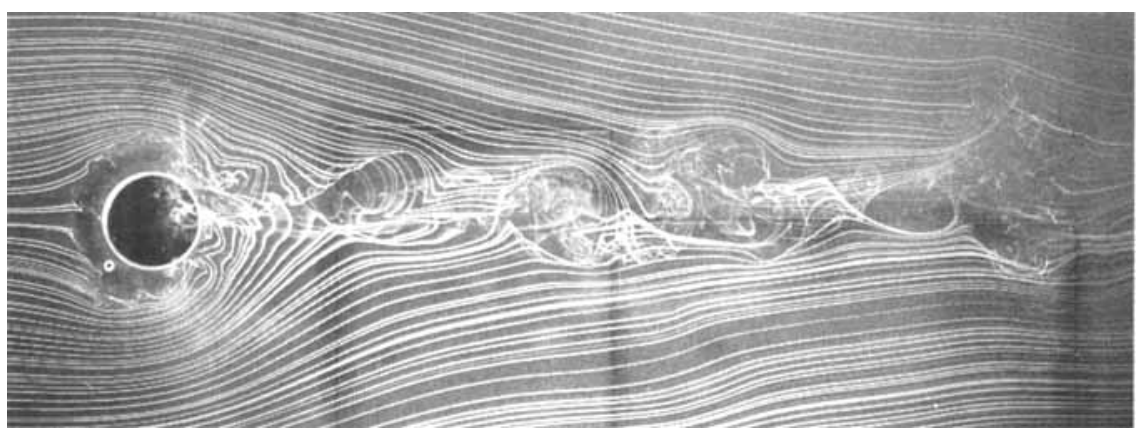

FIGURE 8. Cross-sectional views for $V_{R}=8 . R e_{e f}=3800$. (a) $Z_{s u} / D_{j}=0+;$ (b) $Z_{s m} / D_{j}=0.5$; (c) $Z_{s y} / D_{j}=2.0$. 
(a)

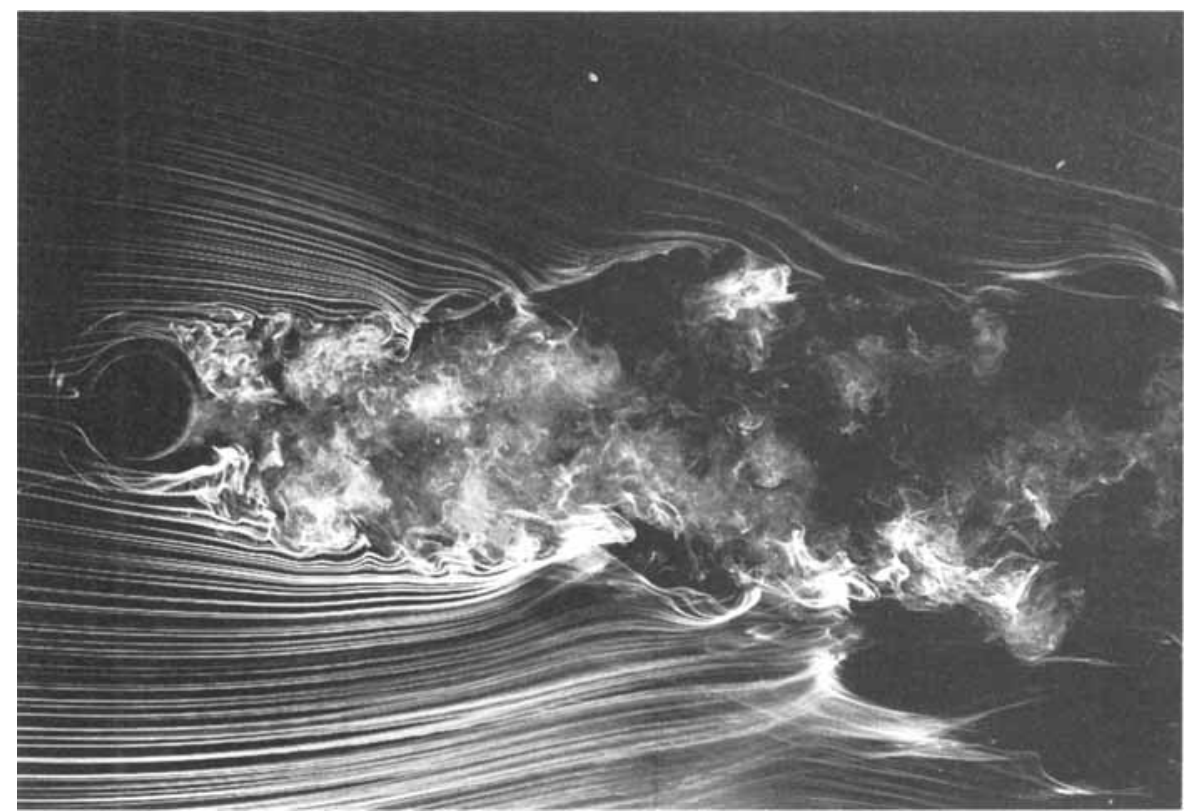

(b)

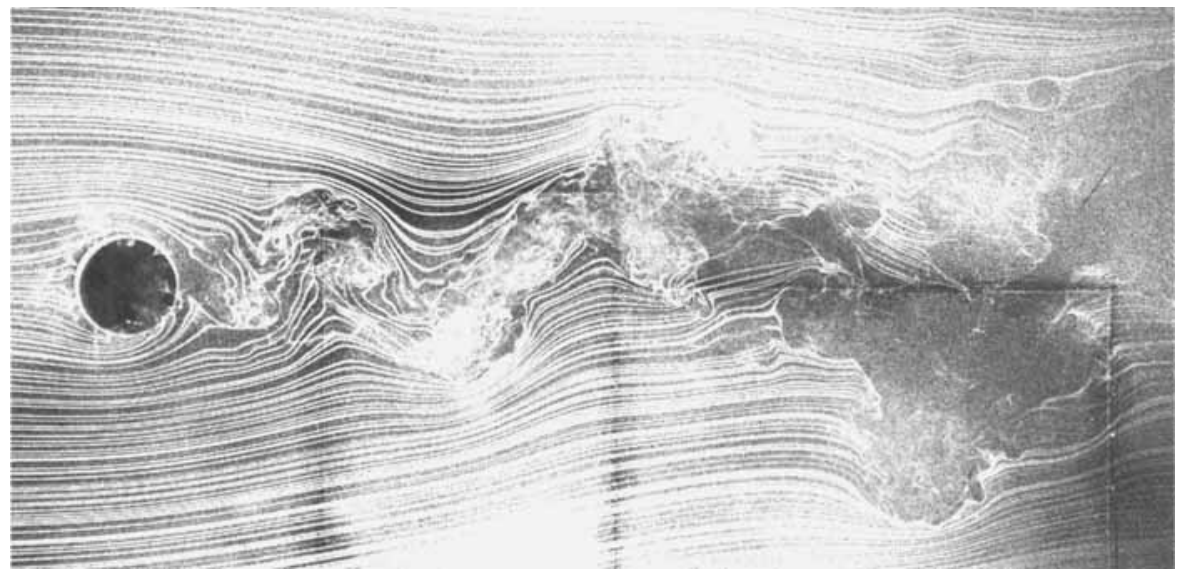

(c)

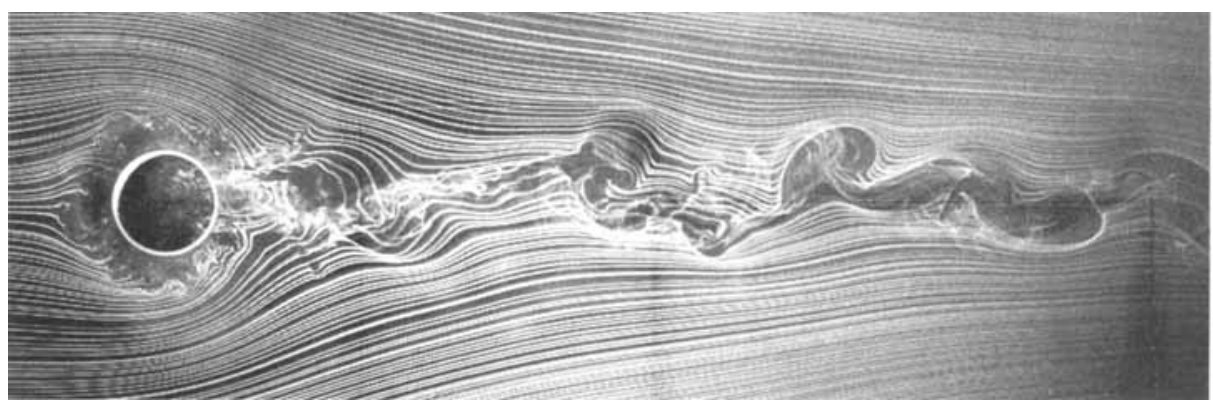

FIGURE 9. Cross-sectional views for $V_{R}=10 . R e_{c f}=3800$. (a) $Z_{s w} / D_{j}=0+;$ (b) $Z_{s u} / D_{j}=0.5$; (c) $Z_{s w} / D_{j}=2.0$. 
Figures 5-9 show sectional views of transverse jet wakes for five different velocity ratios $V_{R}$, ranging from 2 to 10 . The photographs also show the path of the crossflow around the jet and its entrainment into the wake. In each photograph the jet, near the left side of the photograph, issues toward the viewer, and the crossflow is from left to right. The three photographs which comprise each figure represent three different initial smoke-wire positions relative to the crossflow wall. $Z_{s w} / D_{j}$ indicates the plane into which the smoke-wire injects smoke streaklines.

The wake structure near the crossflow wall is visualized in parts $(a)$ of figure $5-9$, in which $Z_{s w} / D_{j}=0+\left(Z_{s w} / D_{j}=0+\right.$ indicates that the smoke streaklines have been injected into the crossflow boundary layer approaching the jet). In each case, at least one horseshoe vortex is seen to wrap around the jet near the orifice. Downstream from the orifice, further structure is seen in the near-wall wake. The wakes for velocity ratios of 4,6 and 8 show structures staggered from side to side, while the wall patterns for $V_{R}=2$ and 10 are significantly different. The portion of the near-wall wake within a couple of jet diameters of the orifice is most 'open' for $V_{R}=2$. At higher velocity ratios, this portion of the flow becomes more closed as the smoke wraps more tightly around the jet. For $V_{R}=10$, it is difficult to discern the larger-scale features that are prominent at lower velocity ratios.

Wake visualizations for two off-wall smoke-wire positions are also shown for each of the five velocity ratios in parts $(b)$ and $(c)$ of each figure. These cross-sectional views clearly show wake structure, again with a qualitative dependence on velocity ratio. For $V_{R}=2$ and 4 (figures $5 b, c$ and $6 b, c$, respectively) the visual widths of the wakes are similar for each of the two off-wall smoke-wire locations. However for $V_{n}=8$ and 10 (figures $8 b, c$ and $9 b, c$, respectively), the visual wake widths appear narrower for $Z_{s w} / D_{j}=2$ than for $Z_{s w} / D_{j}=0.5$. The significance of this $V_{R}$ effect will become clear later.

It is instructive to compare the transverse-jet wakes with analogous views of a circular cylinder wake. Figure $10(a)$ shows the near-wall wake of a wall-mounted circular cylinder, and figure $10(b)$ shows the cylinder wake further from the wall. The cylinder used here had an aspect ratio $A_{R}$ of 6 , chosen to roughly mimic an equivalent penetration distance of a $V_{R}=4$ jet. Comparing these two photographs with the transverse-jet wake photographs reveals telling differences between the wakes. Figure $10(a)$ shows that the near-wall wake of the cylinder is more open than that of the jet, particularly for velocity ratios greater than 2 . The view in figure $10(b)$, at some distance from the wall, is the well-known one, showing the early separation on the cylinder, a wide open near wake and the development of alternating vortex structure, the so-called 'shedding' of vortices. This is in contrast with the jet-wake images, which show the external flow closing around the jet much more completely, little or no open wake (away from the wall), and early formation of vortical structure.

Additional sectional views of jet wakes are shown in figures 11 and 12 . Figure 11 shows, again, some remarkable wake structure typically seen at velocity ratios near 4 and 6 . Note that even though vortices of alternating signs are observed, the vortices do not necessarily lie in a well-ordered array as in the Kármán vortex street. Figure 12 shows wakes at the highest velocity ratio $\left(V_{R}=10\right)$ and crossflow Reynolds number $\left(R e_{c f}=11400\right)$ investigated. The near-wall wake structure of figure $12(a)$ is quite disordered in appearance, as is the case in figure $9(a)$. Clearly evident in this photograph is the fine-scale structure at the edge of the wake along the wall. This structure is common to wakes at this velocity ratio. Above the boundary-layer region, figure $12(b)$ shows a relatively narrow wake with vortices clearly present.

Figures $5-9,11$ and 12 exhibit the presence of vortex structure, in some cases 
(a)

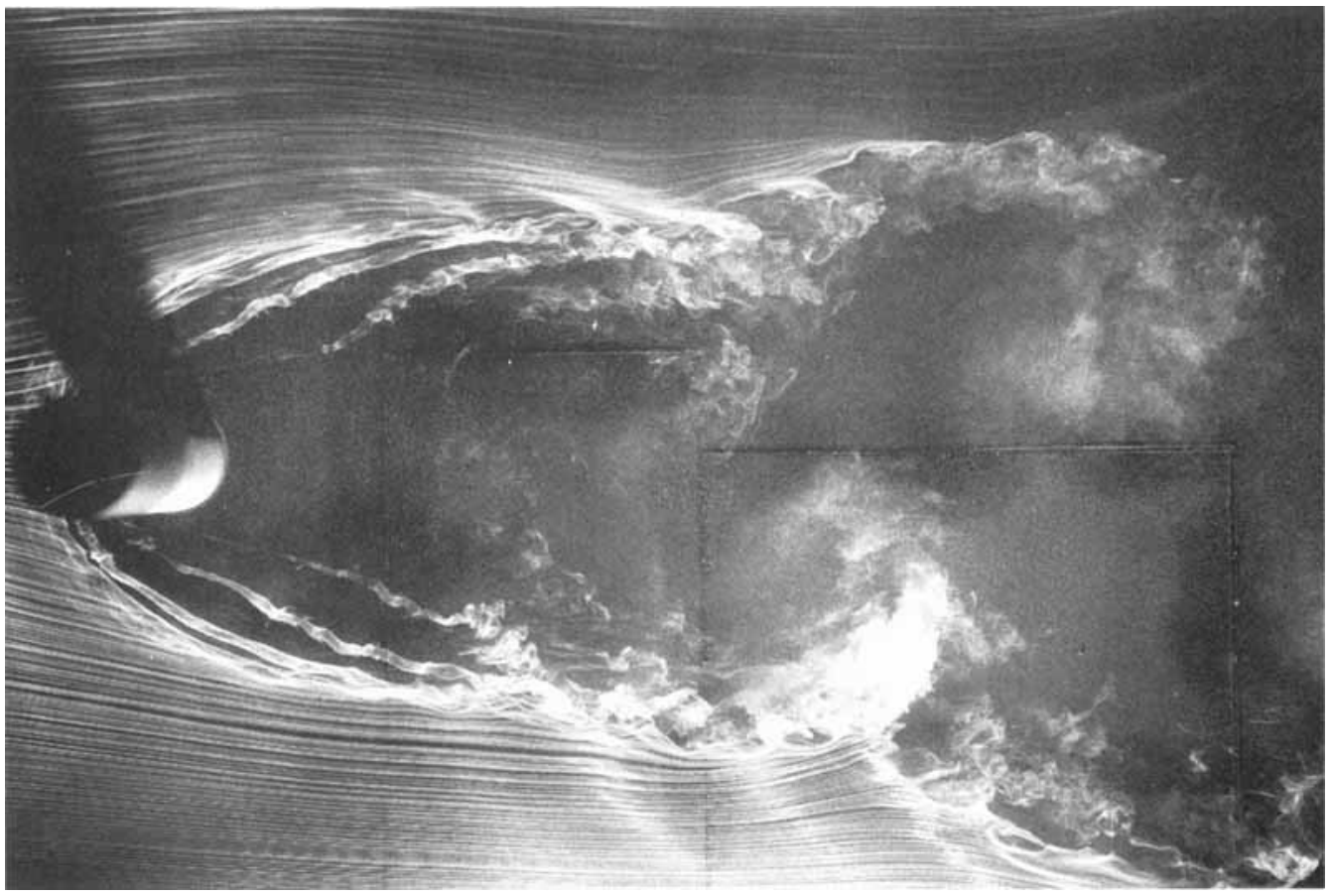

(b)

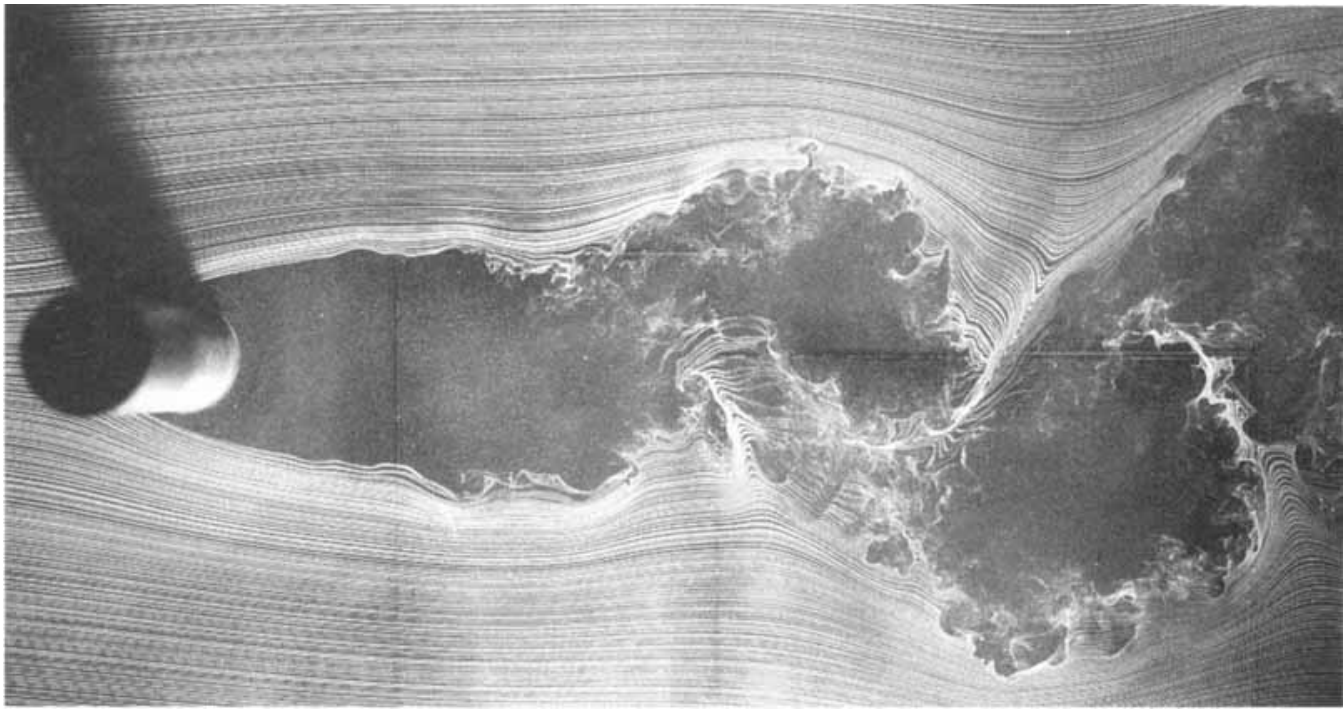

FIGURE 10. Cross-sectional views of a wall-mounted circular cylinder with aspect ratio $A_{n}=6$. In $(a)$ smoke streaklines originate in the crossflow boundary layer, and in (b) they originate half a cylinder diameter away from the wall.

alternating, in the jet wake, reminiscent of but different in some respects from that of a cylinder wake. What is remarkable, however, is that those vortices do not show up in side views in which the jet was visualized by placing smoke into the body of the jet (figure $2 a$ ) or by placing it into the circumferential layer of the jet (figure $2 b$ ). If 
(a)

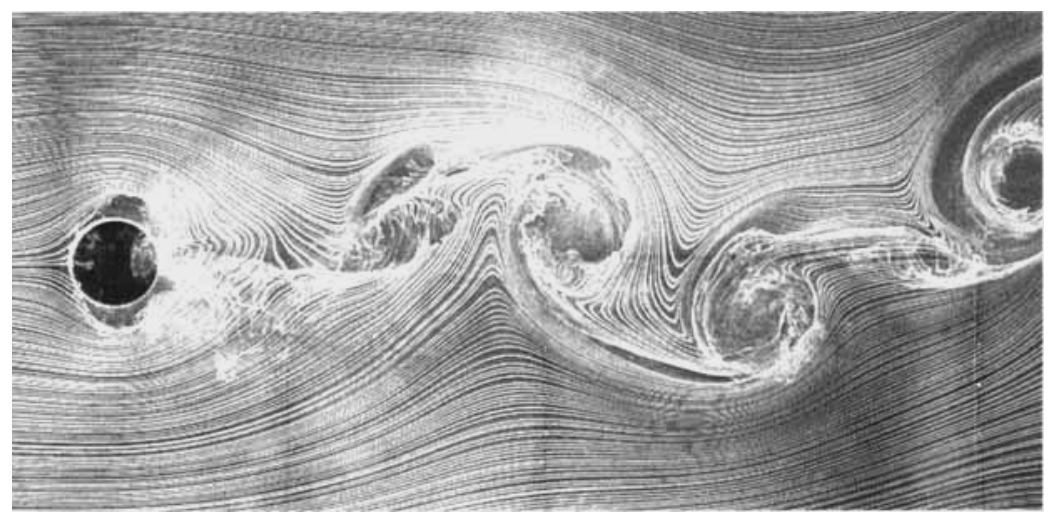

(b)

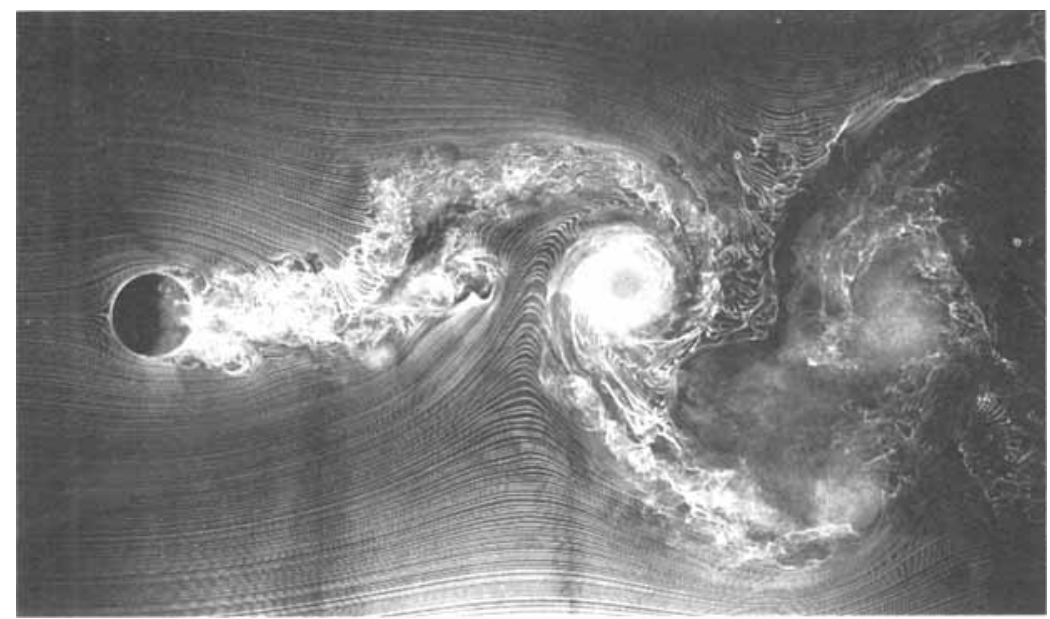

(c)

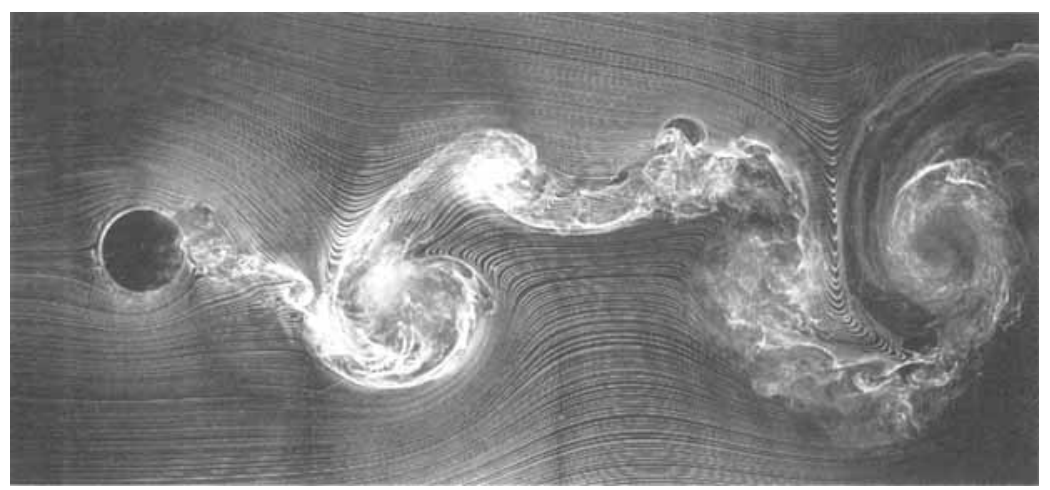

FIGURE 11. Cross-sectional views of jet wakes. $R e_{c f}=7600$. (a) $V_{R}=4, Z_{s q u} / D_{j}=1 ;$ (b) $V_{R}=6$, $Z_{s w} / D_{j}=0.25 ;(c) V_{R} \stackrel{c f}{=} 6, Z_{s w} / D_{j}=0.5$. 
(a)

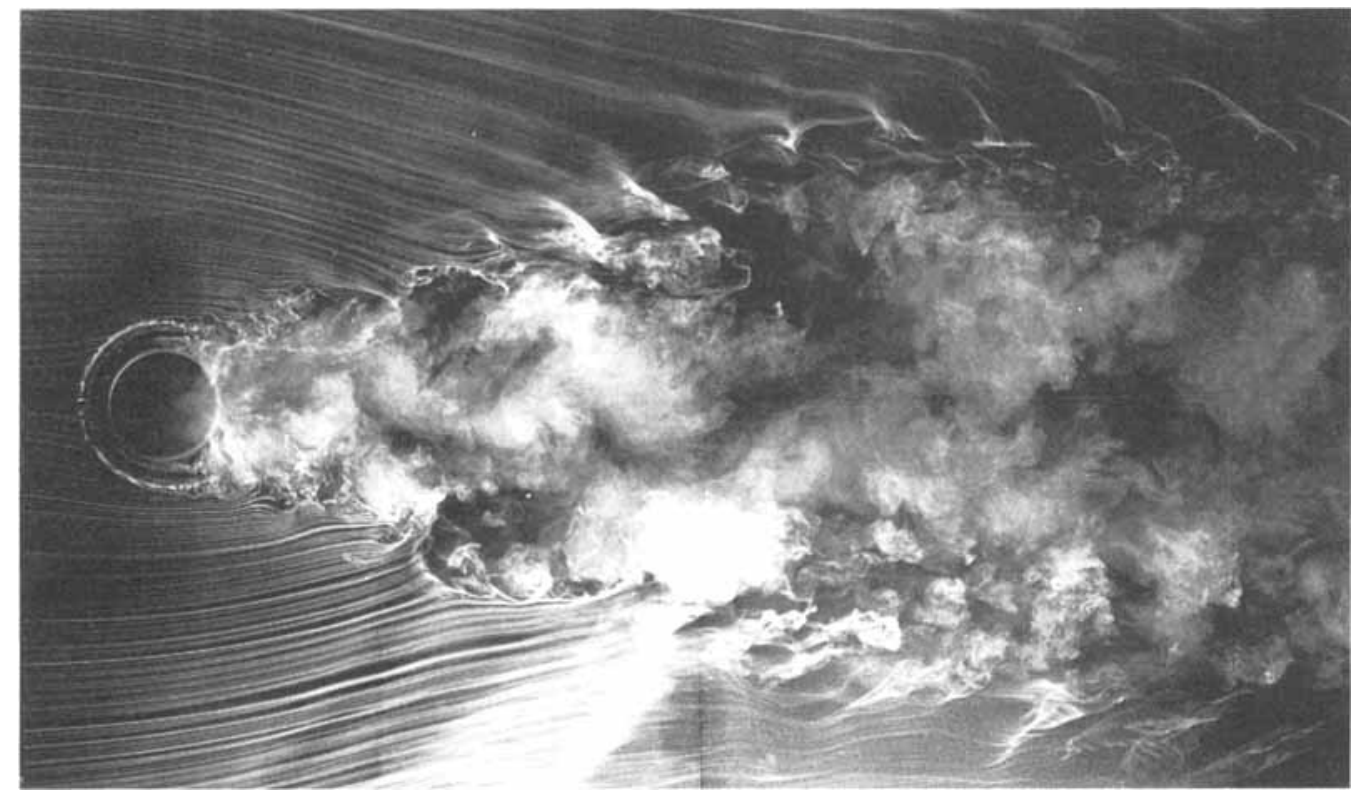

(b)

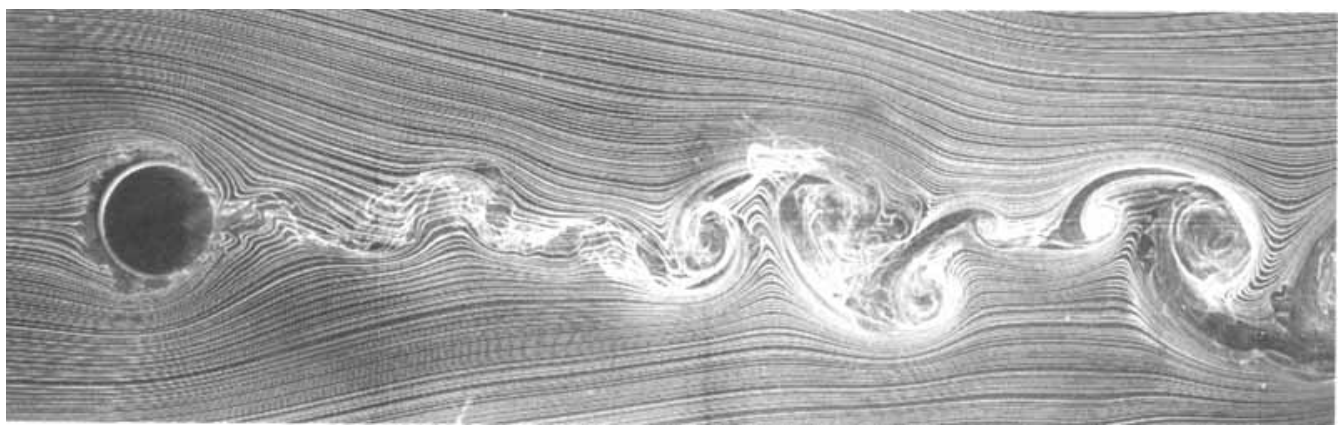

Figure 12. Cross-sectional views of jet wakes for $V_{R}=10$ and $R e_{c f}=11400$. (a) $Z_{s u} / D_{j}=0+$; (b) $Z_{s w} / D_{j}=1$.

vorticity were being shed from the jet circumferential layer then it ought to be accompanied by smoke which should mark the wake vortices that are seen in the crosssectional views. Correspondingly, Kuzo \& Roshko (1984) had visualized the jet-wake vortices in side view by injecting dye not into the circumferential layer but just outside it, from where it was entrained into the wake.

With these various indications that the jet-wake vortex structure does not have its origins in the jet vorticity we speculated that, the only other source of vorticity being the wall boundary layer, the latter must be the source of the wake vortex structures. Thus we were led to the experiment illustrated in figure 13, in which smoke was injected only into the wall boundary layer, upstream of the jet, by a smoke wire placed normal to the plane of the photograph and located just above the wall $\left(Z_{s w} / D_{j}=0+\right)$. (This is the same arrangement as for parts (a) of figures 5-9.) Note also that this technique 
seeds only a thin sheet of the crossflow boundary layer, and thus only a portion of vorticity in the layer is marked. With this arrangement we see in figure 13 that the wake vortices are visualized but the jet is not marked, indicating again that the vorticity in the wake vortices originates in the wall boundary layer, not the jet. As expected, this is in contrast with the wake of a solid cylinder (figure 14): with the same placement of the smoke wire in the wall boundary layer, the wake vortices are not visualized.

The dots drawn on these photographs indicate the outer boundary of the deflected jet, as deduced from the envelopes of visible smoke on photographs like the one shown in figure $2(a)$. Within the portion visualized the wake vortices are generally tilted somewhat with respect to the initial jet direction, and they extend from the crossflow wall to the deflecting jet, lengthening as the jet trajectory takes them further from the crossflow wall, especially at higher velocity ratios. The inferred interaction between the wake vortices and the jet is discussed further in $\$ 6$.

These side-view wake visualizations also suggest a qualitative dependence on velocity ratio. Figure $13(a)$ indicates that the wake structures are not well defined for $V_{R}=2$. As the velocity ratio is increased to 4 and 6 (figures $13 b$ and $13 c$, respectively), the vortices are seen more clearly. Particularly at $V_{R}=4$, the structures are well organized and evenly spaced. Above $V_{R}=6$ there is a change, from wakes whose structures are fairly uniform along their spans to wakes in which dense clumpy structures near the crossflow wall are joined to very thin strand-like structures extending to the deflected jet. At these higher velocity ratios of 8 and 10 most of the smoke, which originates in the crossflow boundary layer, stays within one or two jet diameters of the wall. This dependence on velocity ratio is addressed again, in $\S 6.5$, in the discussion of a formation mechanism.

At the higher velocity ratios of 8 and 10 it appears that the wake vortices are so stretched that smoke only faintly visualizes the thin segments away from the wall. These vortices, or more precisely their induced flow, are more vividly seen if smoke is injected directly in the wake region. This is the case in figure 15 , where the smoke wire was placed in the wake and aligned in the $Z$-direction (the smoke wire can be 'seen' in the photographs since the smoke streaklines begin at its location). In figure 15(a), the smoke wire is placed two diameters into the wake $\left(X_{s w} / D_{j}=2\right)$, and in figure $15(b)$, it is six diameters from the orifice $\left(X_{s w} / D_{j}=6\right)$. These show the thin vortices more clearly at the higher velocity ratios as the smoke is entrained by them.

\section{Wake Strouhal frequencies}

To characterize the jet wakes further it is of interest to examine whether the vortical structures convect past a fixed point at characteristic Strouhal frequencies. It is well known that there is a characteristic Strouhal number for the Kármán-vortex wake of flow past a circular cylinder, and that over Reynolds numbers in the range of the present experiments it is nearly constant. Thus, frequency measurements in the jet wake can provide a further comparison with solid cylinder wakes. We present these results in terms of a wake Strouhal number $S t_{w}$, based on the crossflow velocity, jet diameter and wake frequency.

The degree of repeatability of $S t_{w}$ for fixed parameters is shown in figure $16(a)$, which suggests that, at least for $R e_{c f}=3800$ and $L_{j} / D_{j}=5$, there is a well-defined range of $S t_{w}$ for velocity ratios near the value 4 . There is a distinct change of $S t_{w}$ at $V_{R} \approx 5.5$ and another more variable one near $V_{R}=3$. Figure $16(b)$ shows $S t_{w}$ vs. $V_{R}$ for five combinations of crossflow Reynolds number $R e_{c f}$ and crossflow wall length $L_{j} / D_{j}$. Evidently $S t_{w}$ depends on both of these parameters as well as velocity ratio $V_{R}$, and 
(a)

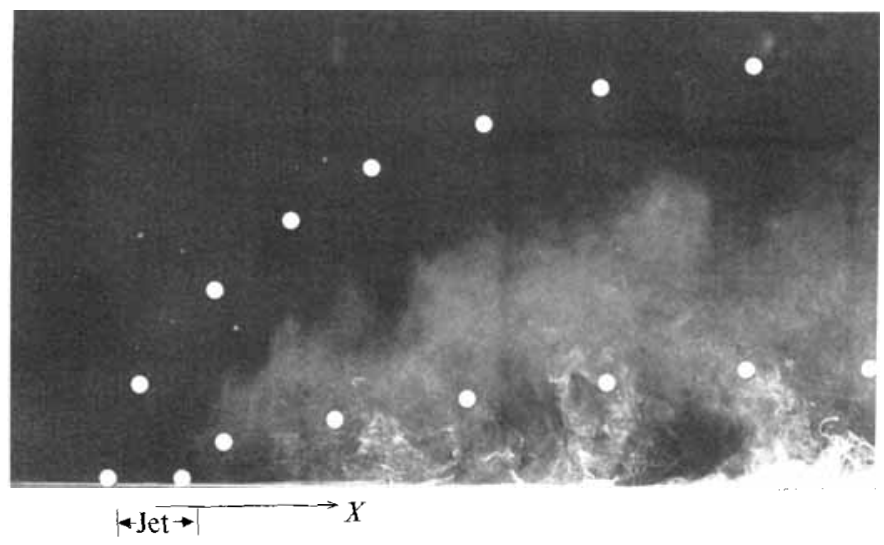

(b)

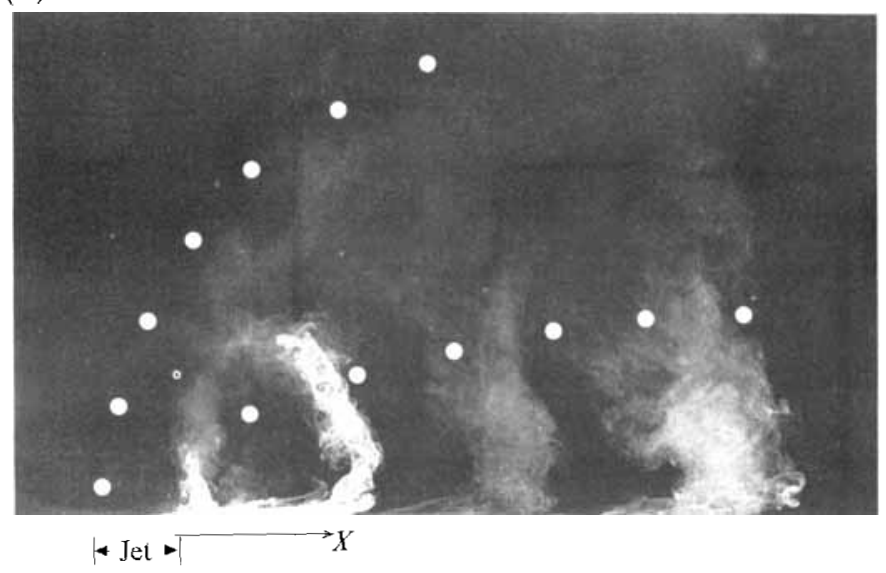

(c)

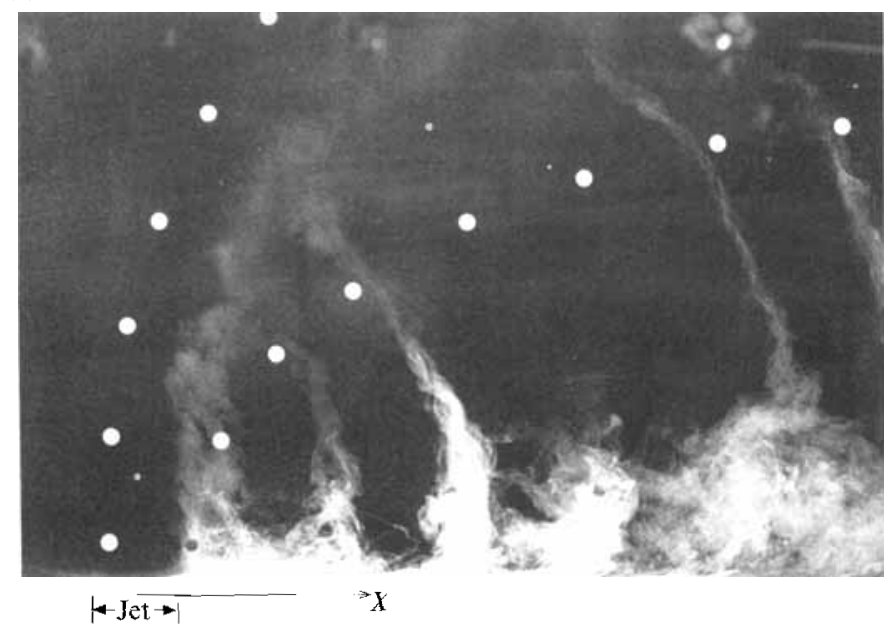

Figure $13(a-c)$. For caption see facing page. 


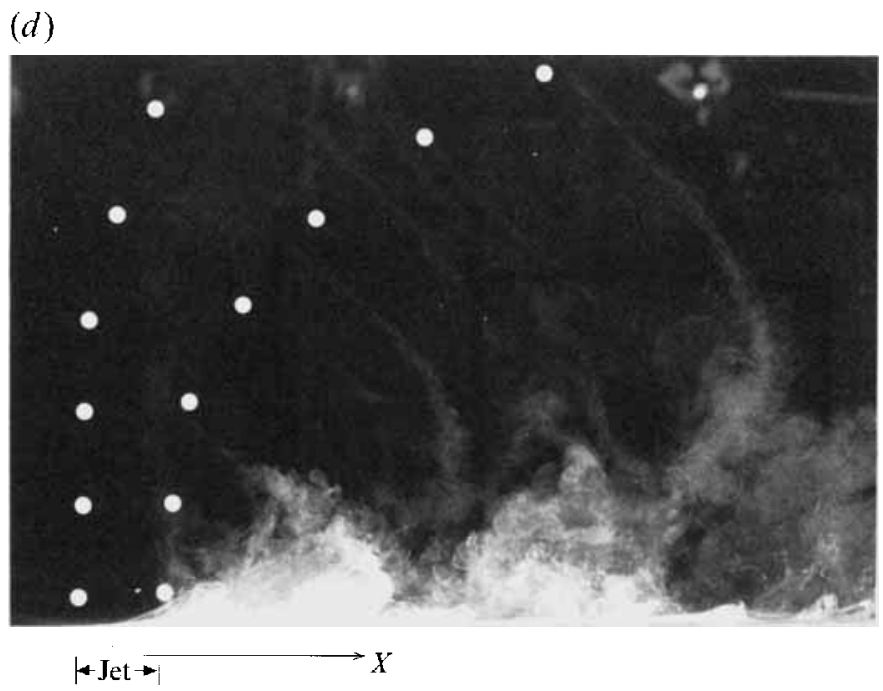

(e)

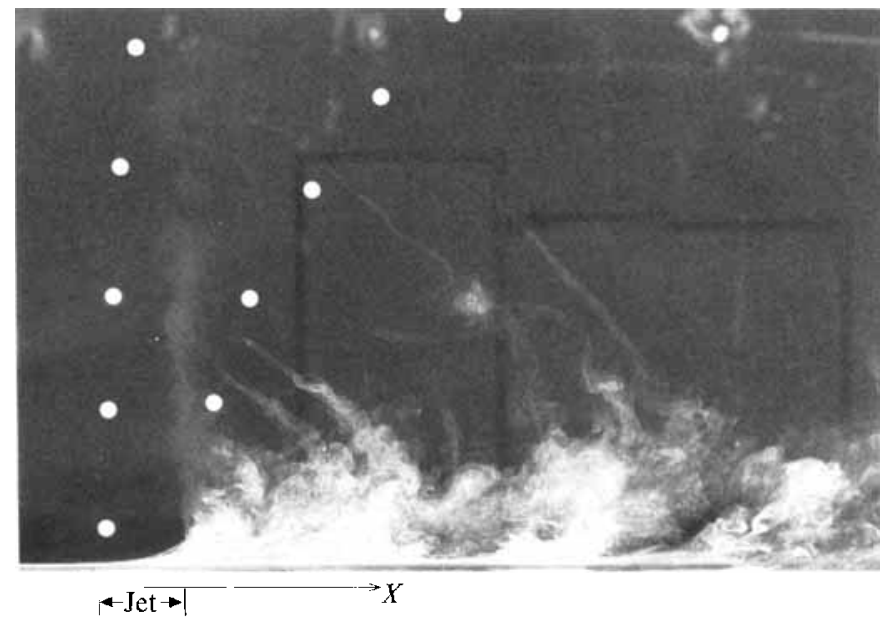

FIGURE 13. Side vicws of wake vortices. Wake structure is visualized by smoke-secding the crossflow boundary layer with a smoke wire $\left(Z_{s u} / D_{j}=0+\right)$. $R e_{c f}=3800$. (a) $V_{R}=2$; (b) $V_{R}=4$; (c) $V_{R}=6$; (d) $V_{l}=8 ;(e) V_{R}=10$.

changes are sometimes rather abrupt. Again, in the range of $V_{R}$ near 4 there is less variability and less dependence on the parameters. The values in this range are near $S t_{w}=0.13$, quite different from the well-known value of about 0.21 for circular cylinders in the same range of Reynolds number. We also noted a range of small variability near $V_{R}=2$, with $S t_{w} \approx 0.16$.

A striking dependence of power spectra on velocity ratio was also observed, with the sharpest spectra measured near $V_{R}=4$ for all crossflow Reynolds numbers and wall lengths. An example is shown in figure $17(a)$. The spectra are least sharp (e.g. figure $17 b$ ) near $V_{R}=3$ and 6 , where there is also greater variability and dependence of $S t_{w}$ on $R e_{c f}$. From our limited data, there are no clear trends with variations in $L_{j} / D_{j}$ and $\delta_{c f} / D_{j}$. However, the variations of the wall boundary layer were not large in this 


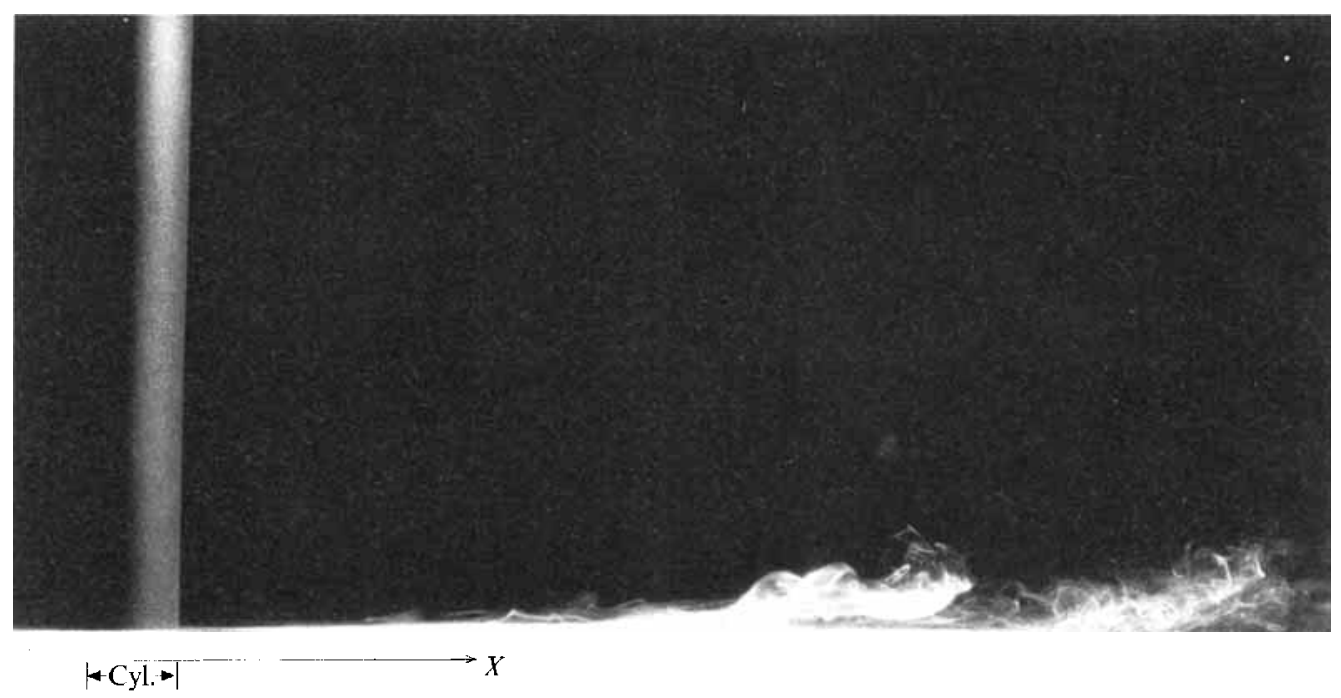

FIGURE 14. Side view of a wake of a wall-mounted circular cylinder when the crossflow boundary layer is tagged with smoke $\left(Z_{s w} / D_{j}=0+\right)$. Contrary to the case of the transverse jet (figure 13), the smoke remains near the wall.

investigation; it is possible that large changes in the crossflow boundary-layer thickness or a turbulent crossflow boundary layer would have different effects.

For comparison there are wake frequency data measured by McAllister (1969), Reilly (1968), McMahon et al. (1971), and Moussa et al. (1977). Those values of $S t_{w}$ are in the same range as the present results. The closest direct comparison is between the data of Moussa et al. for $R e_{c f}=8000, L_{j} / D_{j}=2.7$ and the present data for $R e_{c f}=$ $7600, L_{j} / D_{j}=5$. In particular, their results agree quite well near $V_{R}=4$, and they comment that a change in 'regime' occurs near $V_{R}=3$. Since no elaboration is given, it is not clear whether their observation corresponds to the poorly defined spectral peak and the discontinuity observed near $V_{R}=3$ in the present results (see, again, figure 16).

\section{Wake profiles}

For a further comparison of transverse-jet wakes with wakes of solid cylinders, we measured a few profiles of velocity and total pressure. The results show velocity and total pressure deficits for the jet wakes. Ordinarily a momentum deficit in the wake of a solid body is a measure of the drag force on the body; in a wind tunnel this force is transmitted to its wall. There is no corresponding 'drag' on the transverse jet from its wake. Furthermore, a total pressure deficit in a wake can be a measure of dissipation and, in the case of the solid cylinder, is also connected with its drag. Again, the connection with a transverse jet is not so clear. Since flow visualization indicates that there is transport of boundary-layer fluid into the jet wake, it is possible that the lower momentum and total pressure of that fluid contribute to the corresponding wake deficits. In the light of such issues, it should be of interest to measure these quantities for the transverse jet and try to understand their significance.

Velocity data were obtained with a single-wire hot-wire probe traversed from the centreline $Y / D_{j}=0$ to $Y / D_{j}=3$, where the data asymptote to a free-stream value close to $U_{c f}$. We measured profiles at three downstream distances $X / D_{j}=3.5,5.5$ and 7.5, 
(a)

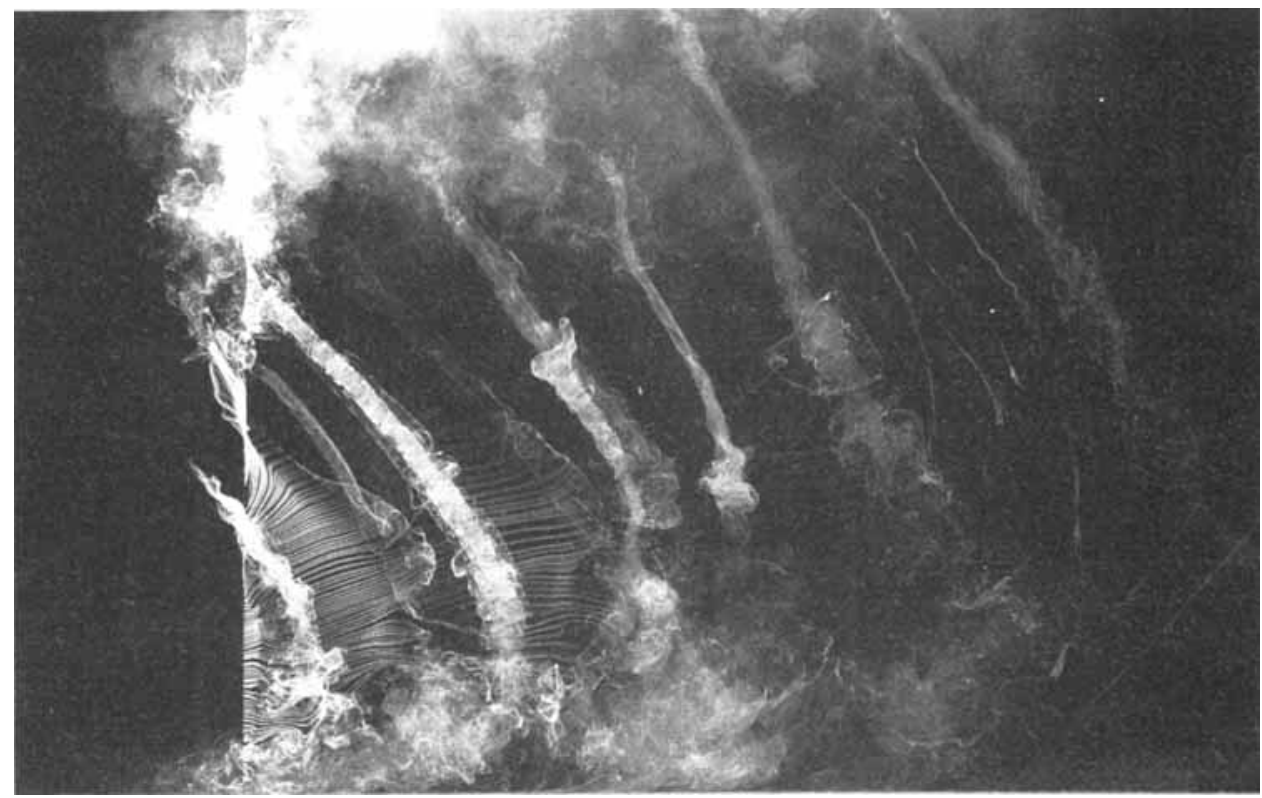

$\rightarrow \mathrm{Jet} \rightarrow_{\text {Smoke wire }} \rightarrow X$

(b)

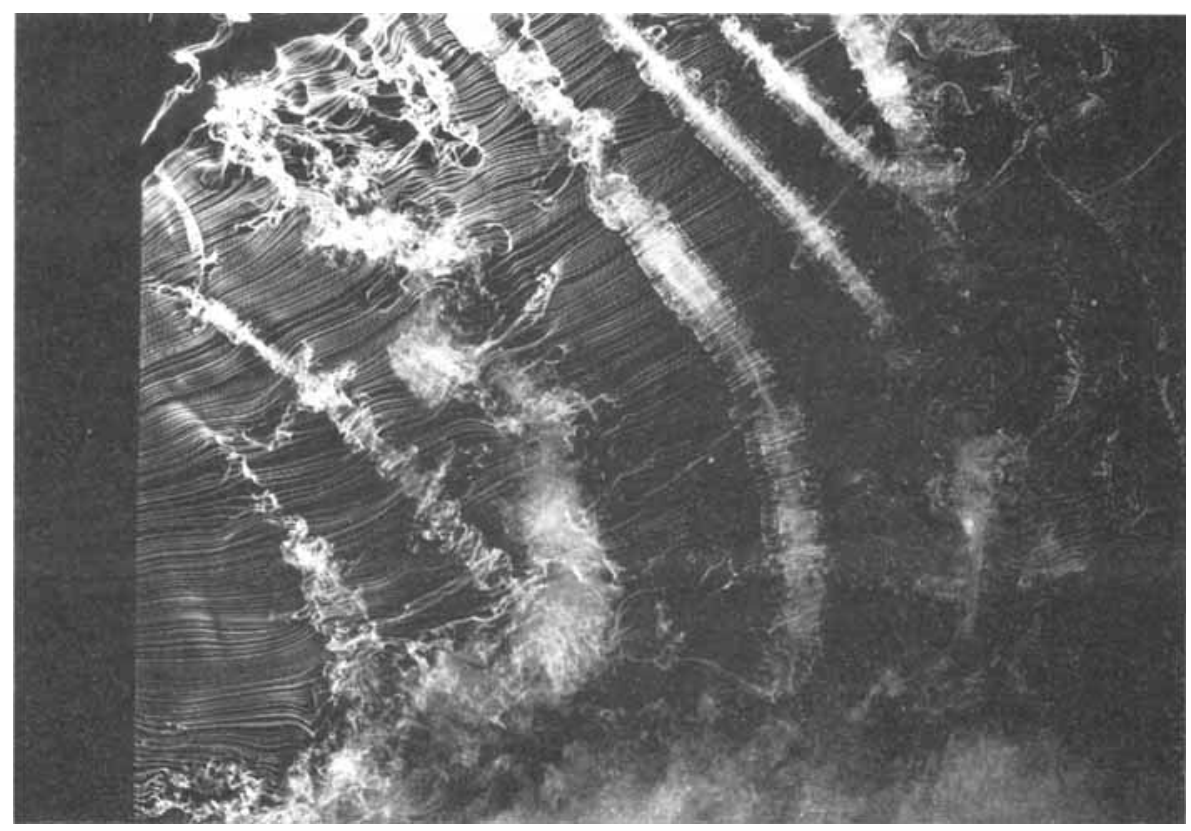

FIGURE 15 . Side views of wake vortices with smoke injected directly into the wake. $(a) V_{R}=8$, $R e_{c f}=3800, X_{s w} / D_{j}=2, Y_{s w} / D_{j}=-0.5 ;$ (b) $V_{R}=10, R e_{c i f}=11400, X_{s w} / D_{j}=6, Y_{s w} / D_{j}^{R}=0$. 

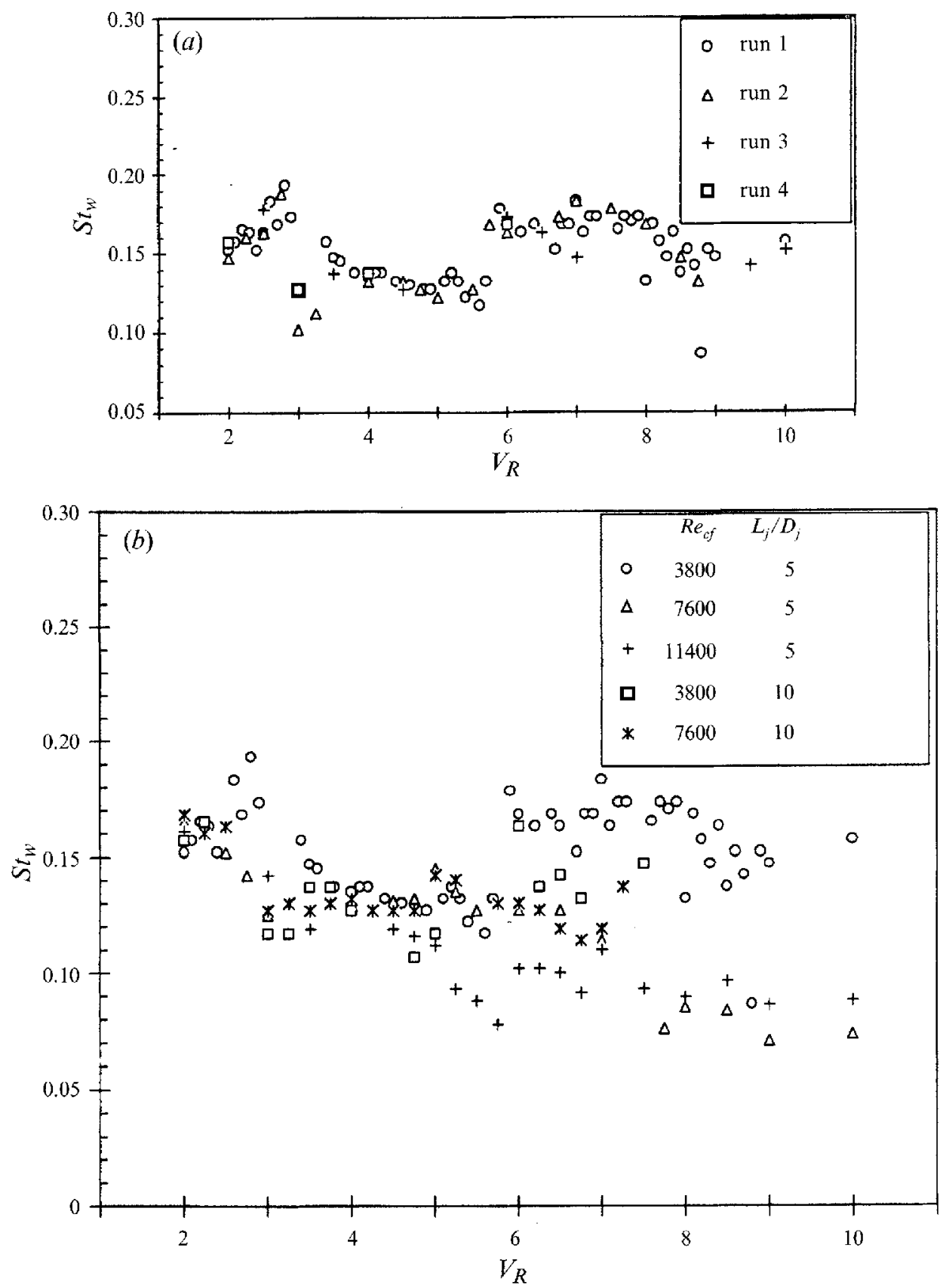

FIGURE 16. Wake Strouhal number $S t_{w j}$. (a) Degree of repeatability of the measurement; $(b)$ data for several combinations of $R e_{c f}$ and $L_{j} / D_{j}$, measured at $X / D_{j}=3.5, Y / D_{j}=1.5$, and $Z / D_{j}=0.5$.

and at three distances from the crossflow wall, $Z / D_{j}=0.5,2.5$, and 4.5 . The maximum r.m.s. velocity fluctuations for the data were generally about $20 \%$ of the crossflow velocity. Such turbulence levels introduce some error to the measured mean velocities. Also, it was observed that there was flow in the $Z$-direction along the axes of the 

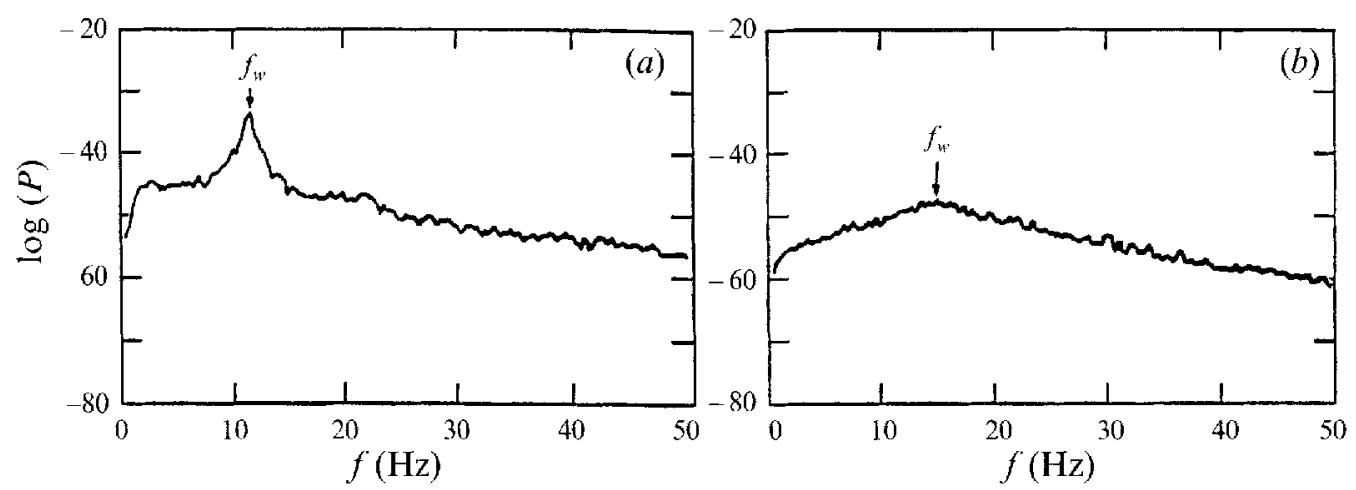

Figure 17. Definition of wake Strouhal frequency $f_{w}$ from power spectrum. (a) Typical of spectra for $V_{t i} \approx 4$ and $(b)$ for $V_{t i} \approx 3$.

vortices. We assume that this does not introduce significant error in the $U$-values since the hot-wire was positioned such that it was least sensitive to flow in that direction. Jetwake velocity profiles for two different wall boundary-layer thicknesses, with $L_{j} / D_{j}=$ 5 and 10 , were obtained for two jet-velocity ratios, $V_{R}=4$ and 8 . Profiles for the wallmounted cylinder were also measured for comparison. Cylinder wake data at $Z / D_{j}=$ 5.5 are not shown since that position is significantly affected by flow around the end of the cylinder, whose aspect ratio $A_{R}=6$.

Figure 18 shows some of the velocity profiles that were measured. The mean streamwise component of velocity is non-dimensionalized by $U_{c f}$. This figure shows jetwake profiles for a relatively thin crossflow boundary layer $\left(L_{j} / D_{j}=5\right)$ and a relatively thick crossflow boundary layer $\left(L_{j} / D_{j}=10\right)$, and cylinder wake profiles. These profiles were measured at $X / D_{j}=5.5$. The velocity deficits are typically greater for the solid cylinder wake than for the transverse jet wake, and this is seen in figures $18(b)$ and $18(c)$. Although the differences between the two sets of transverse-jet wake data are small and in some cases negligible, most of the profiles for $L_{j} / D_{j}=10$ show larger deficits than those for $L_{j} / D_{j}=5$. For instance figures $18(a)$ and $18(c)$ show a slightly larger velocity deficit for the thicker crossflow boundary layer. In figure $18(b)$, the difference is negligible. In figure 19 we show estimates for wake displacement thickness $\delta_{w}$. It is estimated from

$$
\frac{\delta_{w}}{D_{j}} \approx \int_{0}^{3}\left(1-\frac{U}{U\left(Y / D_{j}=3\right)}\right) \mathrm{d}\left(\frac{Y}{D_{j}}\right)
$$

We recognize that this equation is only an estimate; since only the $X$-velocity component, $U$, is used, any transfer of mass in the $Y$ - and $Z$-directions is not taken into account. However, for the purposes of an estimate and in particular for comparing the wakes for the two crossflow boundary-layer thicknesses, equation (1) should be useful. We assume that the mass-transfer contribution from the other two directions is similar in the two cases and would therefore not hurt the comparison.

Figures $19(a)$ and $19(b)$ show $\delta_{w} / D_{j}$ data for wakes of velocity ratios 4 and 8 , respectively. Dependence on distance from the crossflow wall, downstream distance, and boundary-layer thickness is suggested. In particular, both figures show that wake displacement thicknesses are larger for thicker boundary layers, in most cases. This result indicates that the wake may be sensitive to changes in the crossflow boundary layer. If the wake vortices were formed by some shedding-like mechanism only at the 

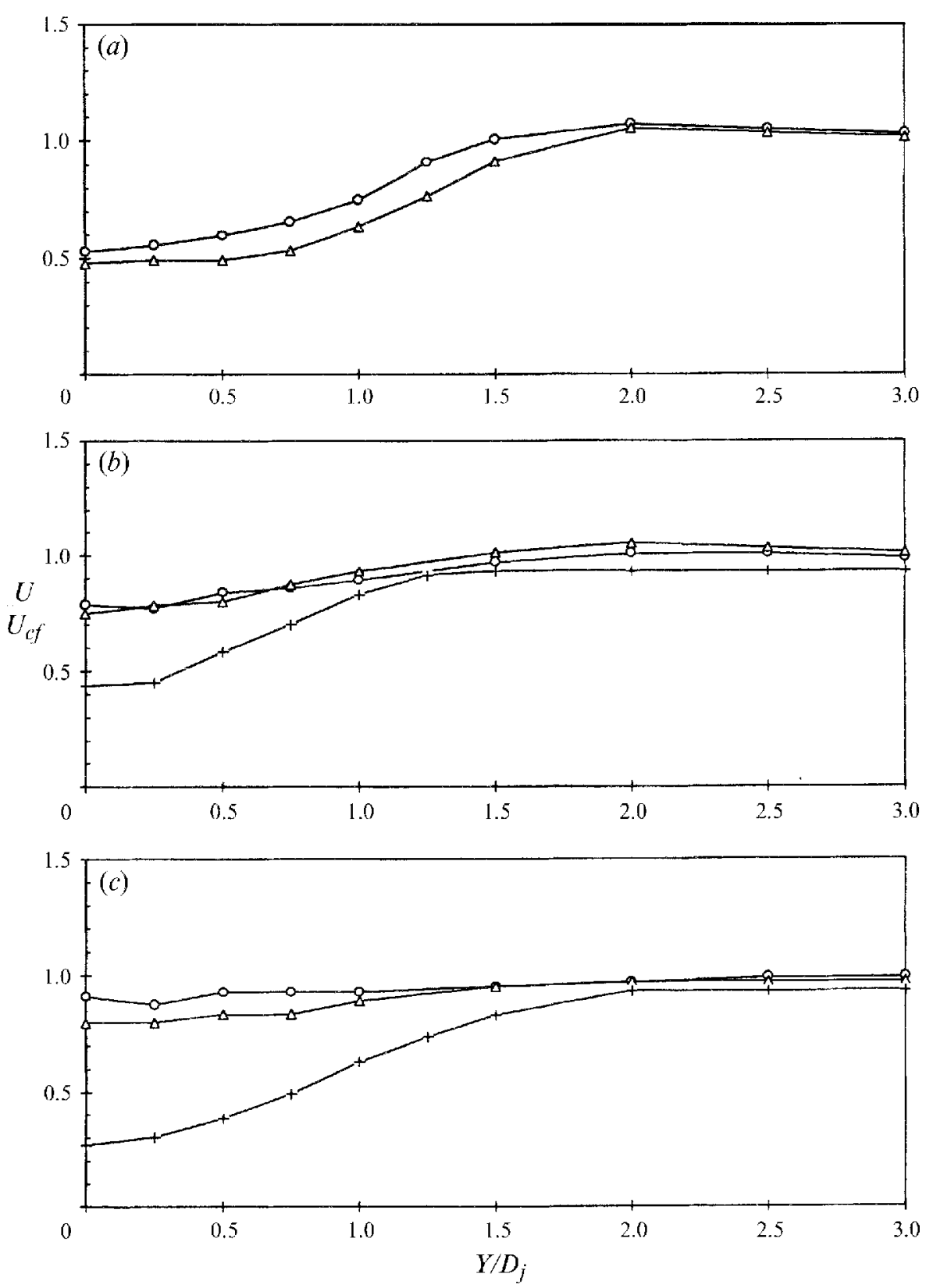

FIGURE 18. Illustrative set of wake velocity profiles for jet of $V_{R}=4$ for two boundary-layer thicknesses $\left(\ominus, L_{j} / D_{j}=5 ; \cdots \triangle^{\cdots}, L_{j} / D_{j}=10\right)$ and for a wall-mounted cylinder $\left(-+-, L_{j} / D_{j}=5\right)$. Downstream location is $X / D_{j}=5.5$. (a) $Z / D_{j}=4.5$; (b) $Z / D_{j}=2.5$; (c) $Z / D_{j}=0.5$.

jet-crossflow interface, we would not expect this sensitivity. However, if the wake forms from vorticity originally in the boundary layer, a dependence on $\delta_{c f}$ may be expected. Furthermore, a comparison of figures $19(a)$ and $19(b)$ shows that $\delta_{w} / D_{j}$ dependence on distance from the crossflow wall is different at $V_{R}=4$ and 8 . Other differences between wakes at lower and higher velocity ratios have been noted previously.

Wake total pressure measurements on the $Y=0$ plane were also obtained, using a 

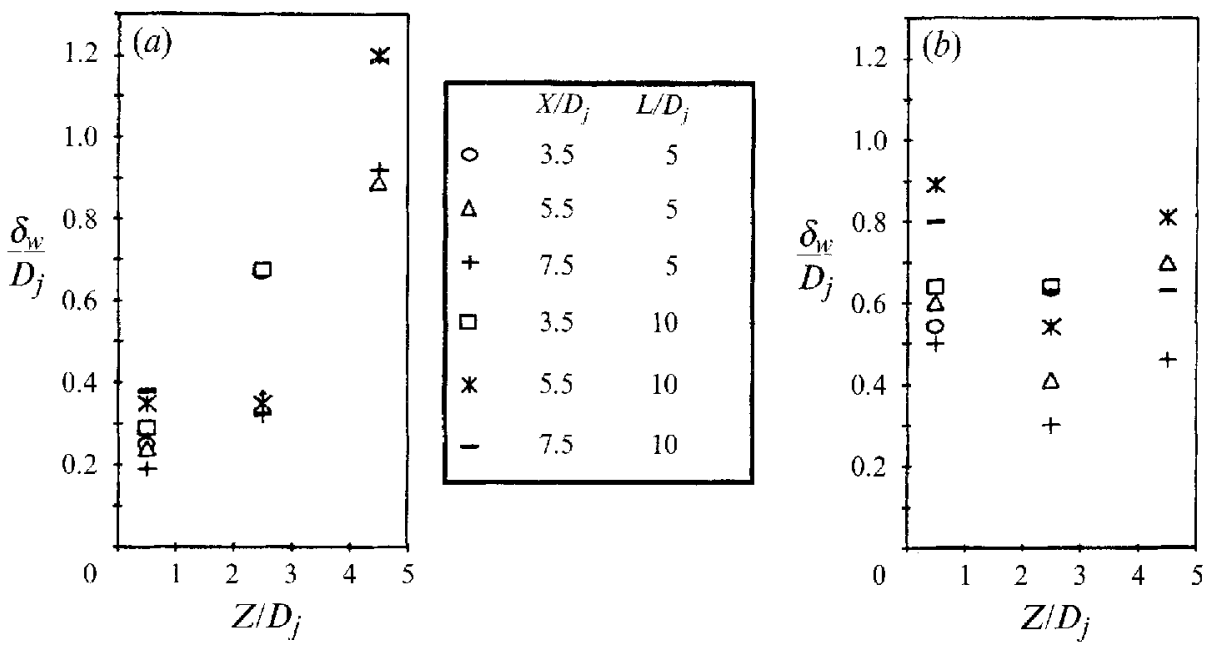

FIGURE 19. Estimates for wake displacement thicknesses. $R e_{c f}=3800 .(a) V_{R}=4 ;(b) V_{R}=8$.

Venturi Kiel probe. Since the total pressures measured with the probe depended (weakly for a Kiel probe) on the alignment with the flow direction, the probe was rotated in the $Y=0$ plane until a maximum value was measured. Results at $R e_{c f}=3800$ are shown in figure 20 for $V_{R}=4$ and 8 jets, and for the wall-mounted circular cylinder. The total pressure coefficient $C_{p t o t}$, defined as

$$
C_{p t o t}=\frac{P_{t w a k e}-P_{t c f}}{\frac{1}{2} \rho U_{r f}^{2}}
$$

is plotted against the downstream distance from the nozzle or cylinder. $P_{i w a k e}$ is the local wake total pressure and $P_{t c f}$ is the crossflow total pressure. The negative values represent total pressure deficit.

Figure 20 shows differences in total pressure trends for the $V_{R}=4$ and 8 wakes and the cylinder wake along the wake centreline. For $V_{R}=4$ (figure 20a) the total pressure deficit increases with distance from the wall. Conversely, at the higher velocity ratio of 8 (figure $20 b$ ) it decreases and then levels off with distance from the wall. The reason for this difference between the two wakes is not clear, but it may be related to differences in wake structure at lower and higher velocity ratios. When seeding the crossflow boundary layer with smoke our flow visualization revealed that the vortical wake structure at $V_{R}=4$ is uniform along its span while at $V_{R}=8$ most of the smoke remains closer to the wall. These observations may explain why the largest total pressure deficit for $V_{R}=8$ is near the wall, assuming the wake structure visualized contains (lower total pressure) boundary-layer fluid. On the other hand, we cannot explain why the total pressure deficit would actually increase with distance from the wall, as it apparently does at $V_{R}=4$, but these relative differences in $Z / D_{j}$ dependence are consistent with the differences shown for wake displacement thickness in figure 19. In particular, note that $\delta_{w}$ increases with $Z / D_{j}$ for $V_{R}=4$.

Total pressure deficit for our cylinder wake lies roughly in the same range and, as should be expected, decreases with distance from the wall. The end effects for the relatively short cylinder are evident. 

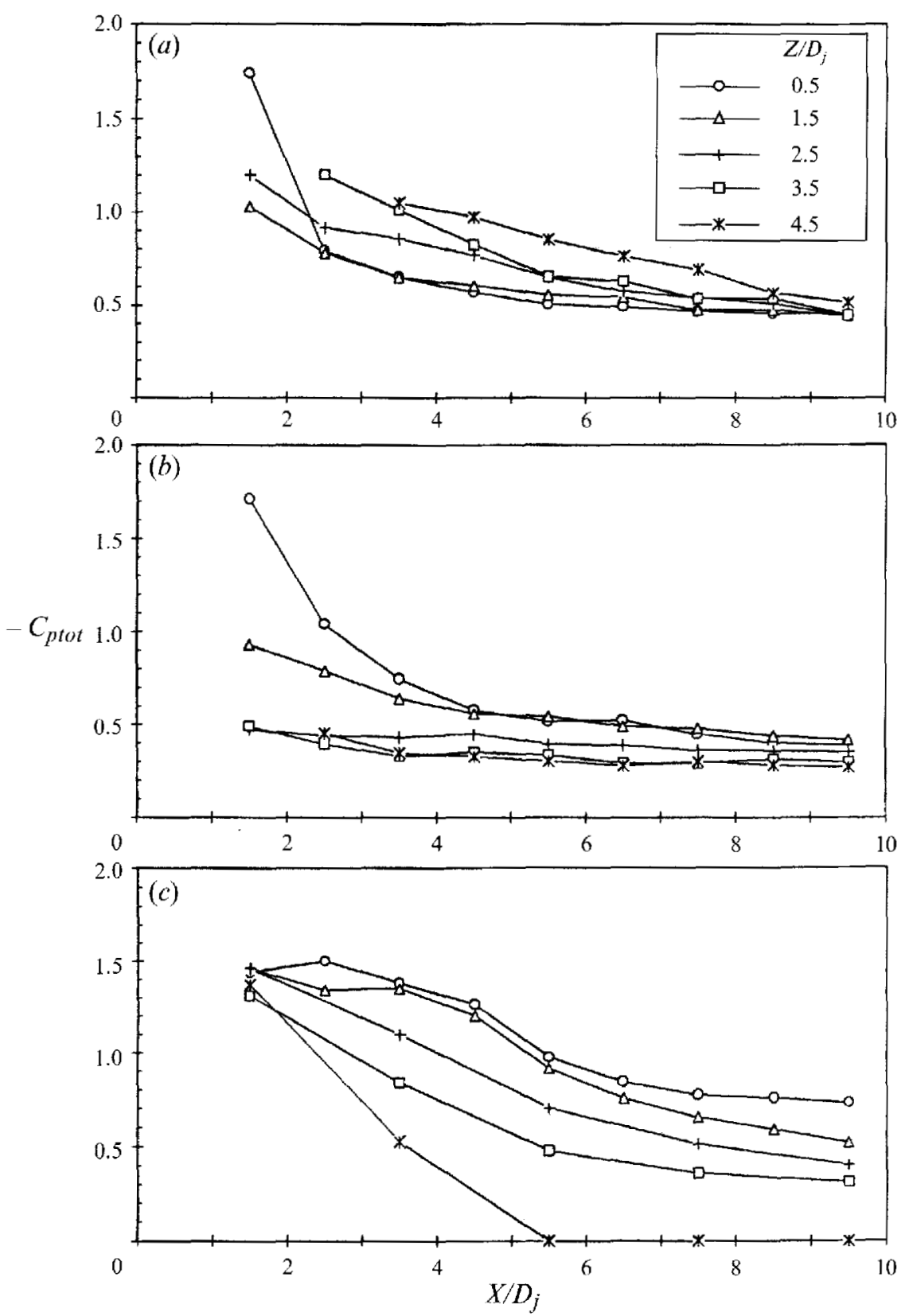

FIGURF 20. Wake total pressure deficits at the wake centreplane $\left(Y / D_{j}=0\right) . R e_{f}=3800$ and $L_{j} / D_{j}=5$. (a) $V_{R}=4 ;(b) V_{R}=8 ;(c) A_{R}=6$ cylinder.

\section{Wake vorticity source and wake formation}

The preceding results, particularly the flow visualizations of figure 13, suggest that the wake vortex structures contain vorticity which has its origin in the wall boundary layer. This raises questions as to why jet vorticity is not shed into the wake and how wall vorticity gets into it. To illuminate such issues, it is useful to review basic concepts of vorticity generation and transport in this section. This will also make clearer why jet wakes cannot form in an analogous manner to cylinder wakes. In particular, we emphasize that there are two aspects that distinguish jet-wake formation from cylinder- 
wake formation. The first concerns the generation of new vorticity or circulation. From kinematic considerations, the transverse jet and solid cylinder each must have a component of $Z$-vorticity as the cross-stream flows around them. For the case of the cylinder that vorticity is generated at its surface but for the transverse jet we argue that vorticity cannot be generated at the interface of the jet and crossflow. Instead, that vorticity must convect, stretch, turn and diffuse from its source at a solid surface. The second aspect which distinguishes the formation of jet and cylinder wakes is the transport of vorticity into the wake. This aspect is distinct from the first in that it addresses the transport of vorticity, conceivably that of $Z$-vorticity at the jet-crossflow interface, into the wake even though it was not initially generated at that interface. Our results show, however, that the transport of vorticity for the wake is from the crossflow boundary layer and not from the jet-crossflow interface. In this section we also introduce the 'separation events' which are a result of crossflow boundary-layer separation and which lead to the jet's wake formation.

\subsection{Vorticity generation}

To understand the origin of the vortical wake structures and to determine their source of vorticity requires an understanding of vorticity generation. In what follows, the appropriate vorticity transport equation for the present flow is introduced and the ways in which vorticity can be generated are discussed.

For a flow such as this one, namely Newtonian, barotropic, isothermal, incompressible and with no body forces, the vorticity transport equation is given by

$$
\frac{\mathrm{D} \omega}{\mathrm{D} t}=\omega \cdot \nabla u+\nu \nabla^{2} \omega .
$$

As emphasized by Morton (1984), this equation does not explicitly contain any vorticity source or generation terms; it shows only that existing vorticity is convected, stretched, turned, and diffused. The second term, the vortex stretching and turning term, is non-zero only in three-dimensional flows and is necessarily zero at all stationary solid boundaries. The third term allows for the inter-diffusion of vorticity of opposite sign and for the transport of vorticity across streamlines.

Since equation (3) does not have an explicit source term, new circulation can only enter a flow through imposed initial conditions and/or wall boundary conditions; in the terminology of Morton, there are no sources of 'new' vorticity within the flow. This is a fundamental point when considering the formation of the transverse-jet wake vortices. A distinction between new vorticity and vorticity which results from internal processing (stretching, turning and diffusing) of vorticity which is already in the flow is implied. For instance, the second term in equation (3) can produce a new component of vorticity by turning pre-existing vorticity. This is not new vorticity; it is rather the processing of vorticity which already is present. New vorticity effects a local change in flow circulation, in contrast to pure turning or stretching of vorticity which conserves circulation in the sense of Kelvin's theorem. New vorticity, as the term is used here, is synonymous with adding local positive or negative circulation to the flow.

Since vorticity can only enter the flow through imposed initial conditions and/or wall boundary conditions, one can define a vorticity flux out of a wall as $\hat{n} \cdot J_{0}$, where $J_{0}=$ $-\nu(\nabla \omega)_{0}$ is the vorticity flux tensor at the wall, and $\hat{n}$ is the wall-normal unit vector. It can be shown (Wu \& Wu 1993) that for non-accelerating and non-rotating surfaces

$$
\rho \hat{\boldsymbol{n}} \cdot \boldsymbol{J}_{0}=-\hat{\boldsymbol{n}} \times(\boldsymbol{\nabla} p)_{0}-\hat{\boldsymbol{n}}\left(\hat{\boldsymbol{n}} \cdot\left(\boldsymbol{\nabla} \times \boldsymbol{\tau}_{\mathbf{0}}\right)\right)+\left(\hat{\boldsymbol{n}} \times \boldsymbol{\tau}_{0}\right) \cdot \boldsymbol{\nabla} \hat{\boldsymbol{n}} .
$$

(Incompressible flow and the absence of viscosity gradients are assumed in the 
derivation of this equation.) $\hat{n} \cdot \boldsymbol{J}_{0}$, the vorticity flux out of the wall, essentially represents the rate of inflow of vorticity per unit wall area.

The first term on the right-hand side of (4) is the vorticity source term due to a wall pressure gradient; the vorticity produced is tangent to the wall. The role of this term is well known, but it is often incorrectly considered to be the sole source of vorticity at solid boundaries. This would be generally true only in two-dimensional planar flows, when the second- and third-term contributions are identically zero. Therefore, vorticity generation analysis in two dimensions cannot be generalized to three dimensions; the contributions from the second and third terms of (4) would be lost.

As vorticity tangent to a surface is produced, it diffuses away from the solid surface to enter the flow. Wu \& Wu call this the 'ascending mechanism' of introducing vorticity and state that for two-dimensional, attached, and steady flows this pressure gradient term is the main contribution to the vorticity flux out of a wall.

In three-dimensional flows, the second and third terms on the right-hand side of (4) are, in general, non-zero. The second term accounts for the gradient of wall-normal vorticity from a wall shear stress $\boldsymbol{\tau}_{0}$ with a non-zero $\boldsymbol{\nabla} \times \boldsymbol{\tau}_{0}$ wall-normal component. Since vorticity at a wall must be tangential to the surface, the wall-normal component of vorticity is zero at a wall. Immediately above the wall, however, a wall-normal component of vorticity can exist.

With spiral flows oriented normally to and above a wall, such as is the case near the intersection of the crossflow wall and the wake vortices, $\left(\hat{\boldsymbol{n}} \cdot\left(\boldsymbol{\nabla} \times \boldsymbol{\tau}_{\mathbf{0}}\right)\right)$ is non-zero and subsequently this term can be significant. In fact, the analyses of $\mathrm{Wu}, \mathrm{Gu} \& \mathrm{Wu}$ (1987) and Wu \& Wu show that the normal-vorticity production term can be significant near separation lines. (As will be discussed in $\$ \S 6.4$ and 6.5 , separation of the crossflow boundary layer is important for the wake.) They conclude that this process is responsible for the large wall-normal vorticity associated with a "horn vortex' or a 'tornado-like vortex'. Wu \& Wu refer to this phenomena as a 'turning-up mechanism' since it involves the turning-up of vortex lines from the solid boundary.

The third term on the right-hand side of (4) accounts for the effects of wall curvature that has a component transverse to the wall shear stress direction. As with the pressure gradient term, this term produces vorticity tangent to surfaces. The vorticity diffuses away from the wall to enter the flow. In a sense, this is a correction to the pressure gradient term to account for the surface curvature transverse to the flow direction.

\subsection{Solid-cylinder wake vorticity and implications for transverse-jet wakes}

Since it is often assumed that the wake of the transverse jet forms in a manner similar to that of the solid cylinder, it is timely to recall what is known about the generation of vorticity and wake structure for a cylinder wake. The favourable pressure gradient on the upwind side of a circular cylinder generates vorticity for its own wake, of one sign on one side and of opposite sign on the other side. The first term on the right-hand side of (4) is the pertinent one. The unfavourable (positive) pressure gradient on the downwind side causes the boundary layer to separate. The separation and shedding process ultimately transports the vorticity, which was generated at the cylinder wall and which initially diffused away from the wall, into the wake. Figure $10(b)$ shows that the crossflow separates from the cylinder in this way. The separating streaklines are seen coming off the top and bottom sides of the cylinder. But, for the transverse jet flow, visualization shows no analogous separation of the crossflow fluid as it passes over the jet body. Compare, for example, figure $10(b)$ with figures 5-9, parts $(b)$ and $(c)$. There is a striking difference between the wakes, particularly just downstream of the jet and cylinder. The separating streaklines are seen coming off the sides of the 
cylinder, a situation quite different from the case of the jet where the streaklines are nearly closed all the way around.

For the incompressible and barotropic flow here, the sources of circulation are at solid boundaries or walls. In the transverse jet there are, therefore, two possible sources of vorticity for the vortical wake structures which have been observed, namely the boundary layer within the jet nozzle and the boundary layer on the crossflow wall.

In the often-held view that formation of jet-wake vortices is by 'shedding', similar to that from circular cylinders or other solid objects, there is the implication that the source of vorticity for the wake structures is new vorlicity generated at the interface between the jet and the crossflow. But there is no such mechanism for the generation of new vorticity in the case of homogeneous flow around a jet. This does not necessarily imply that $Z$-vorticity is absent at the jet-crossflow interface but only that its origin is different from that at a solid cylinder. That is, the jet's own shear-layer vorticity, originating at the jet nozzle wall, may be processed so that components of $Z$-vorticity are convected into the interface.

The interaction of the jet and crossflow is an interaction between homogeneous fluids, differing only in total pressure. The cylinder wake results from interaction between a solid body and a fluid. Another possibility is interaction between dissimilar fluids, such as gases of different densities or a liquid and a gas, where there exists a mechanism for new vorticity generation. For instance, Jacobs (1992) studied the interaction between shock-wave-generated airflow and a transverse jet of lighter gas (helium). The interaction is such that vorticity is created at the interface between the air and helium by baroclinic torque. The vorticity transport equation (3) would in this case contain the baroclinic source term.

The source of vorticity for the wake of a jet in homogeneous crossflow and the formation of the wake structure are discussed in detail in $\$ \$ 6.36 .5$.

\subsection{Tracking the flow vorticity}

Smoke can be used to track vorticity carried by the boundary layer from within the nozzle and by the boundary layer on the crossflow wall. Smoke is considered to be a suitable marker of vorticity for the present purpose. Owing to the relatively large particle mass of smoke (compared to the mass of air molecules), the diffusivity of smoke is much lower than the molecular diffusivity of air. Cimbala (1984) estimates the ratio of smoke diffusivity to molecular diffusivity to be of order $10^{-5}$. Since the Schmidt number represents the ratio of viscous to molecular diffusivity and is of order unity for air, the ratio of the viscous diffusivity to smoke diffusivity for air (the effective Schmidt number) is of order $10^{5}$. Therefore vorticity diffuses much faster than smoke does; hence we assume that once the vorticity is tagged, the smoke mainly marks the cores of vortices. Diffusion acts to spread vorticity away from the cores faster than smoke.

The suitability of using smoke as a vorticity marker is helped by the high Reynolds numbers of the flows. The diffusion timescales for both smoke and air are long compared to the convective timescales. On the timescale for convection of the structures through the photographs' fields of view, diffusion of both smoke and vorticity should be visually insignificant.

Figure 21 shows two side views of the flow field at $V_{R}=4$ and $R e_{c f}=3800$. In both photographs, the jet issues from the bottom left-hand side of the photograph. Compare figure $21(a)$, where the jet fluid (part of which is the boundary layer within the nozzle) is tagged by seeding the jet supply with smoke, with figure $21(b)$ where the crossflow boundary layer is tagged with smoke. In figure $21(a)$, a well-defined deflected jet is seen with no presence of smoke nor, apparently, of jet fluid in the wake. Conversely, in 
(a)

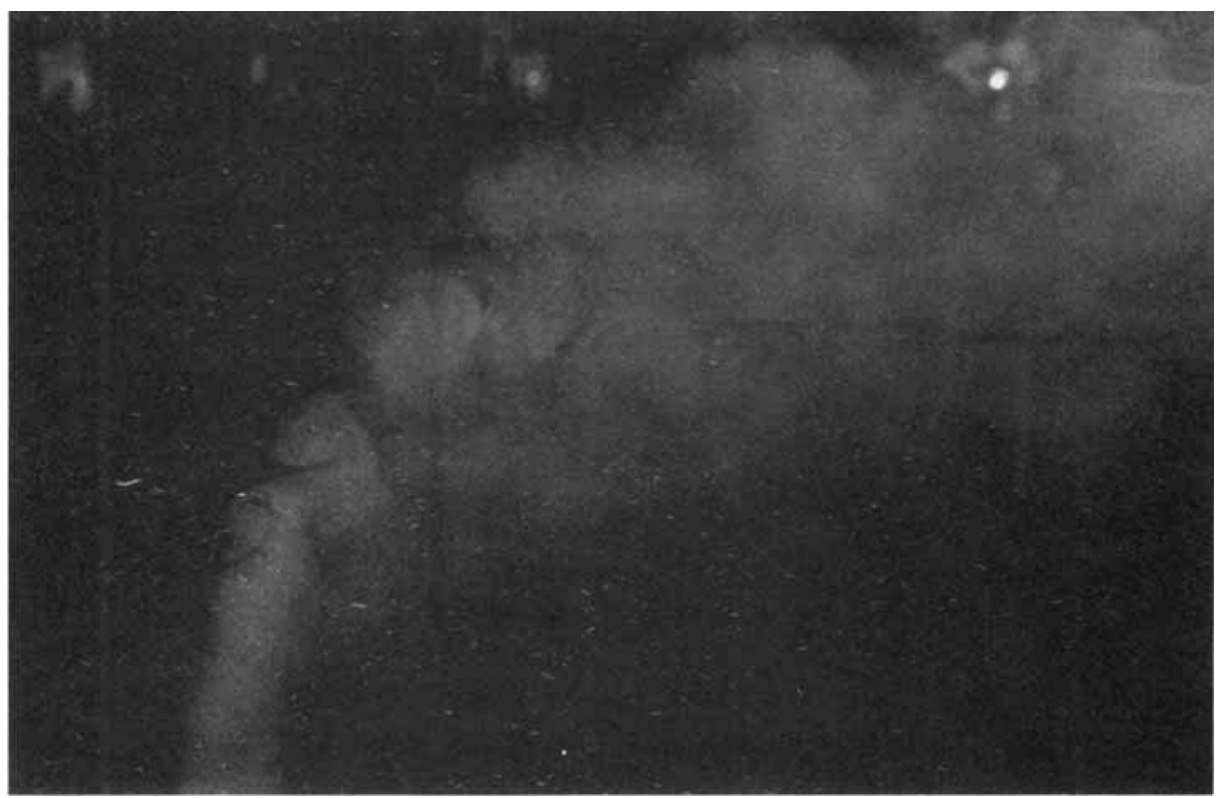

$\leftrightarrow \mathrm{Jet} \rightarrow \longrightarrow X$

(b)

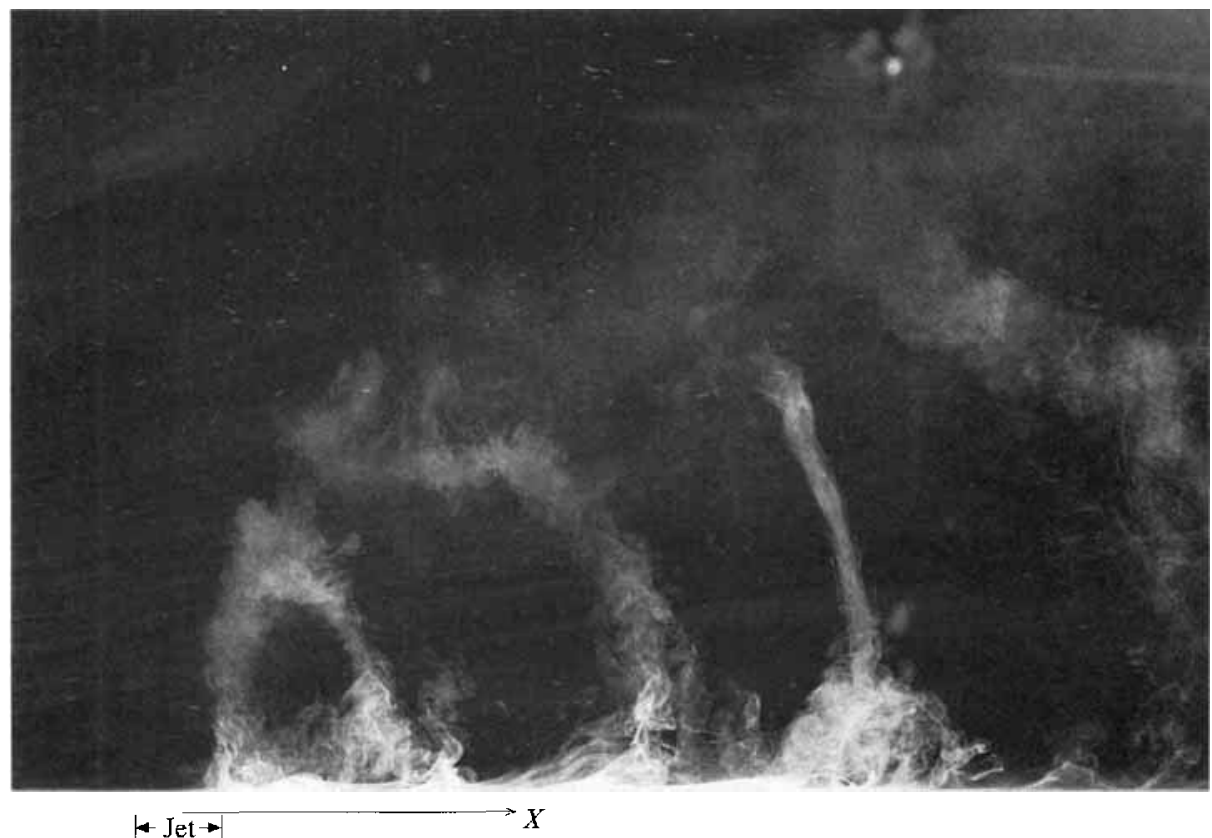

FiguRe 21. Smoke tags the two possible sources of vorticity. In (a) the jet (and therefore the nozzle boundary layer) vorticity is tagged, and in $(b)$ smoke tags the crossflow boundary-layer vorticity $\left(Z_{s u} / D_{j}=0+\right) . V_{R}=4$ and $R e_{c f}=3800$. Note that wake vortices are visualized only when the crossflow boundary layer is tagged. 
(a)

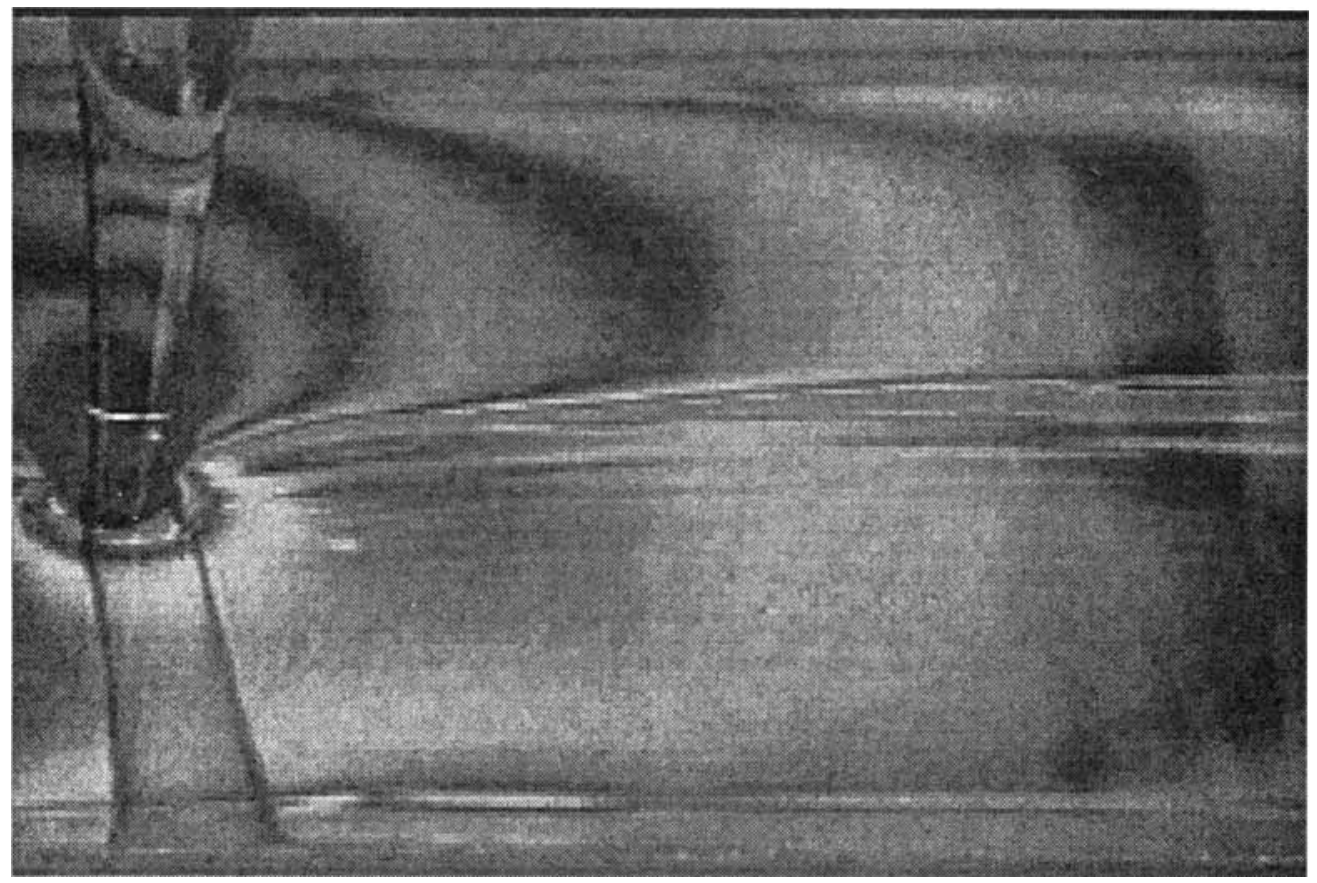

(b)

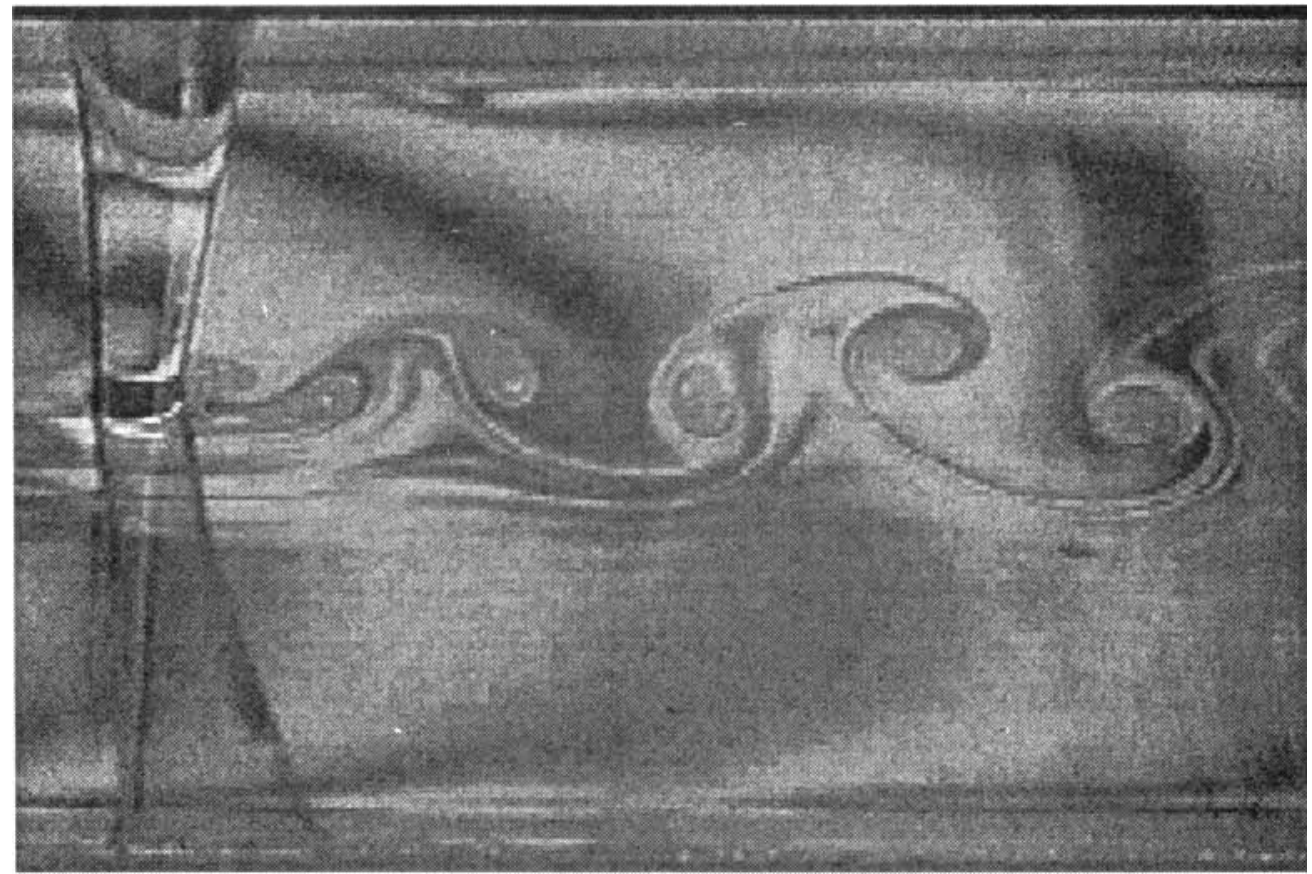

Figure 22. Flow visualization by soap film. (a) A water jet injected through the soap film shows no structure in the wake; $(b)$ the jet nozzle inserted through the soap film sheds vortices. 
(a)

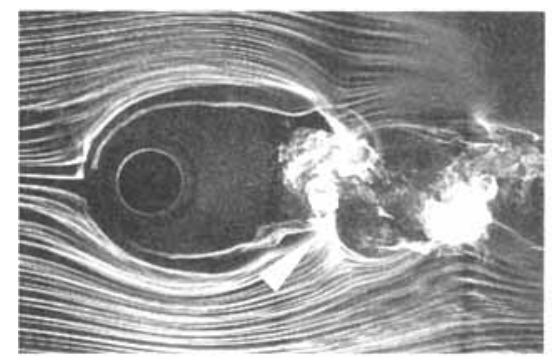

(b)

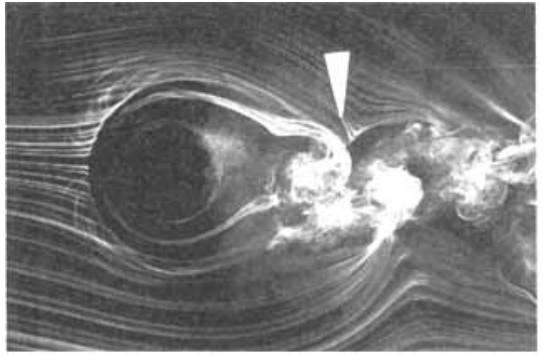

(c)

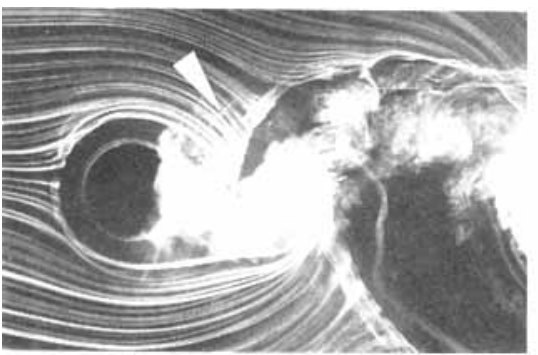

(d)

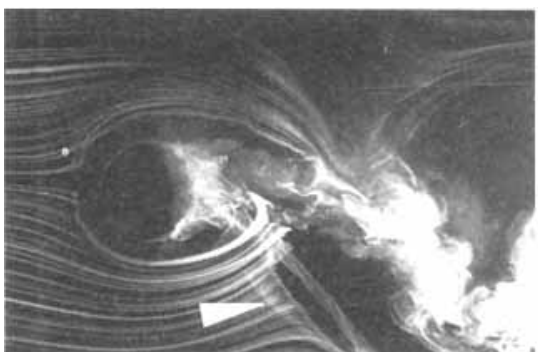

(e)

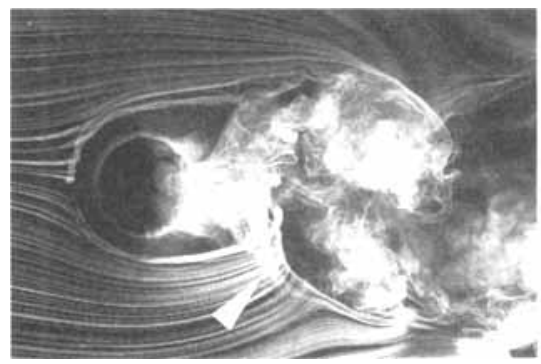

(f)

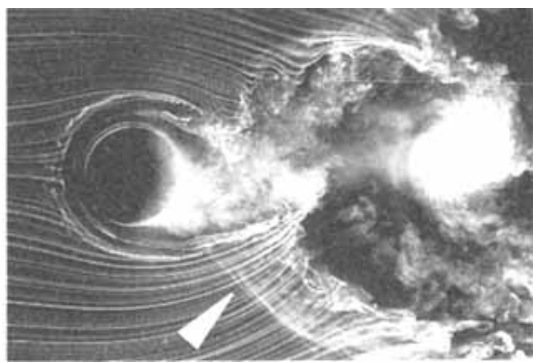

(g)

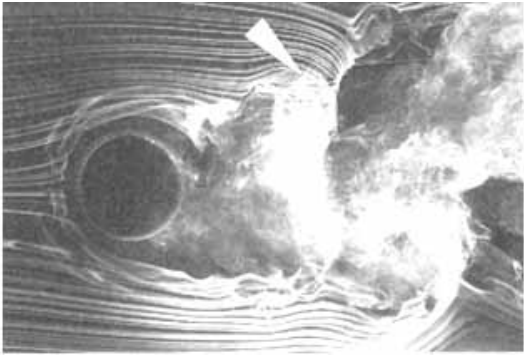

(h)

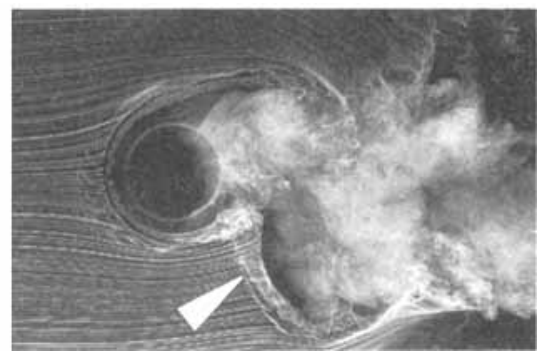

(i)

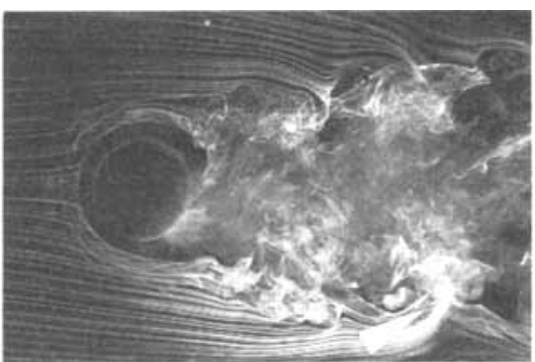

(j)

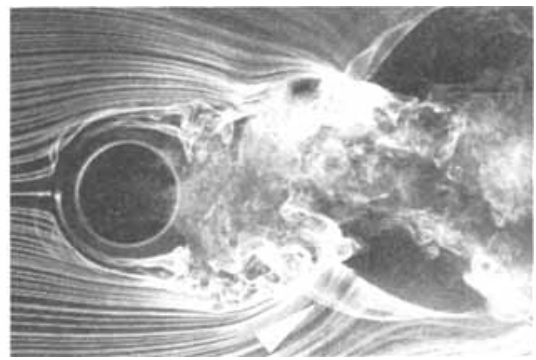

FigurF $23(a-j)$. For caption see facing page. 
figure $21(b)$, much of the smoke finds its way into the wake vortices. Here the wake is strongly illuminated by smoke which could have come only from the crossflow boundary layer. Analogous comparisons can be made for the whole range of $V_{k}$ from 2 to 10 (Fric 1990), and figure 13 shows side views like that of figure $21(b)$ for several velocity ratios. These results suggest that nozzle vorticity does not contribute to wake vorticity. Not only does the jet not act like a solid cylinder, not generating any new vorticity, it also does not appear to 'shed' any of its vorticity into the wake. The wake does not contain visible jet fluid. That smoke originating in the crossflow boundary layer, and therefore marking its vorticity, leaves the wall and visualizes the wake vortices indicates that the source of wake vorticity is the boundary layer on the crossflow wall.

For a comparison of figure $21(b)$ to a circular cylinder wake, refer again to the side view of the wake of a wall-mounted cylinder in figure 14. Here, as in figure $21(b)$ for the transverse jet, the smoke wire is situated such that the crossflow boundary layer is tagged with smoke. The smoke stays relatively close to the crossflow wall and the wake vortices are not visualized, as one may expect, since the source of the cylinder wake vorticity is not the crossflow boundary layer.

These results suggest that eliminating the crossflow boundary would also eliminate the wake vortices since their source of vorticity is no longer present. This was recently accomplished (Fric \& Gharib 1990) by using a soap film 'wind tunnel', where a jet of water was injected transversely through a thin flowing soap film. Since the soap film essentially creates a two-dimensional crossflow (Gharib \& Derango 1989), the crossflow wall and its cffects were eliminated from the problem and the twodimensional interaction of a jet and crossflow was visualized (figure $22 a$ ). The results showed that the soap-film flow closes around the jet and no wake vortices nor structure of any kind are observed downstream of the jet. In contrast, when a solid cylinder is placed transversely through the soap-film flow, the familiar separation and vortex shedding are observed (figure $22 \mathrm{~b}$ ). The vortex-shedding case was also demonstrated by Couder \& Basdevant (1986), who towed cylinders through stationary soap films.

To see jet vorticity entering the wake region would not contradict the vorticity generation theory; transport of the jet's own vorticity (not newly generated as on a cylinder surface) by diffusion or some 'peeling-off' process is possible but our observations do not show this to be the case. Similarly, the papers of Keffer \& Baines (1963), Kamotani \& Greber 1974) and Chassaing et al. (1972) show photographs of transverse jets seeded with smoke. In each case, only the deflected jet is observed, and no smoke is seen in the wake region. Furthermore in Kamotani \& Greber's (1972) study of a heated transverse jet, temperature contours showed that the excess heat downstream of the orifice was confined to the deflected jet and did not, apparently, contaminate the wake with heat. Recently Lozano et al. (1994) have reported results which show a small amount of jet fluid entering a portion of the wake region. In their study 'fingers' of acetone, which was seeded into the jet, were detected in the portion of the wake close to the jet. We do not believe that their results contradict our results or conclusions. Firstly, jet fluid in their wakes was 'visible' only after exponential enhancement of the fluorescence data from the acetone seeding; only a very small amount of the jet fluid made its way into the 'fingers'. Secondly, their photographs show this behaviour only at velocity ratios greater than 10 and not at those studied

FIGURE 23. Near-wall flow structure just downstream of the jet. 'Separation events' and their accompanying roll-ups are identified by the arrows. $Z_{s z:} / D_{3}=0+$. (a) $V_{R}=2 ;$ (B) $V_{n}=3 ;(c) V_{R}=$ $4 ;(d) V_{R}=5 ;(e) V_{R}=6 ;(f) V_{R}=6 ;(g) V_{R}=8 ;(h) V_{R}=8 ;(i) V_{R}=9 ;(j) V_{R}=10$. $R e_{c f}=3800$ in $(a-e),(g),(i),(j) ; R e_{e f}=11400$ in $(f)$ and 7600 in $(h)$. 
here. Most importantly, the wake vortices we have described here clearly extend from the crossflow wall to the jet, while the fingers observed by Lozano et al., extend only a small distance from the jet.

\section{4. 'Separation events' in the crossflow boundary layer}

To this point we have shown evidence leading to the conclusion that the crossflow boundary layer supplies the wake with its vorticity. We now discuss a possible mechanism by which vorticity leaves the wall to enter the wake region. From the wake visualizations and vorticity gencration discussion it is clear that the process is threedimensional, involving separation and turning of vortex lines. The laminar boundary layer initially carries primarily spanwise $(Y)$ vorticity while the wake vorticity is seen to be predominantly in the $Z$-direction.

Upon closer inspection of the near-wall flow, two predominant structures resulting from separations near the jet orifice are evident. Consider the close-up photographs shown in figure 23, where smoke is within the crossflow boundary layer $\left(Z_{s w} / D_{j}=\right.$ $0+$ ). Upstream of the jet, a portion of the boundary layer separates and forms at least one horseshoe vortex. The boundary-layer streaklines, outside those which get rolled into the horseshoe vortices, i.e. those further away from the centreline, flow around the sides of the jet and then appear to separate on the orifice's lee side. The photographs of figure 23 each indicate, with arrows, a vortical structure which has rolled up after separation. We postulate that such 'separation events', as we shall refer to them, are the genesis of wake formation.

As is the case with the wake structure, the near-wall flow visualizations show a qualitative dependence on velocity ratio. In fact at $V_{R}=2$ separation events are difficult to identify, relative to higher velocity ratios. This is consistent with our previous observations of poorly defined wake structure for $V_{R}=2$ (see figure $13 a$ ). Also consistent with the previotis results is the clarity of the separation events for $V_{R}=4$. Recall that the wake structures are relatively well-organized, evenly spaced, and have sharper spectra at $V_{R}=4$. In figure $23(c)$, for example, two separation events are in fact visible - one marked with an arrow and one further downstream and on the other side of the centreline. Particularly at $V_{R}=4$, and at some other velocity ratios albeit with less regularity, these separation events appear to form on one side of the jet and then on the other in an alternating manner. This unsteadiness is reminiscent of vortex shedding from a solid cylinder but in this case vorticity is 'shedding' from the crossflow wall. Separation events are still clearly identifiable at $V_{R}=6$, while at velocity ratios greater than eight they are often somewhat less distinct.

Characteristic (Strouhal) frequencies associated with the separation events were compared with those of the wake vortices in order to make a quantitative connection between them. Photographs such as those shown in figure 23 were used to locate separation events. A hot-wire probe was then positioned near them to obtain the power spectrum of the velocity fluctuation there. The spectral peak frequencies $f_{s e p}$ are thus associated with the event. Separation-event Strouhal numbers $S t_{\text {sep }}$ at various velocity ratios and at three crossflow Reynolds numbers are compared in figure 24. ( $S t_{w}$ data shown in this figure were previously included in figure 16.) The agreement between $S t_{w}$ and $S t_{s e p}$ is very good at each $R e_{c f}$. This correspondence between the characteristic frequencies of the crossflow boundary-layer separation events and those of the wake structures indicates an intimate connection between them. Together with the near-wall flow visualization, it supports the conclusion that these separation events are an important element in the formation of wake structure.

To illustrate the mechanism by which the separation events occur, a comparison 

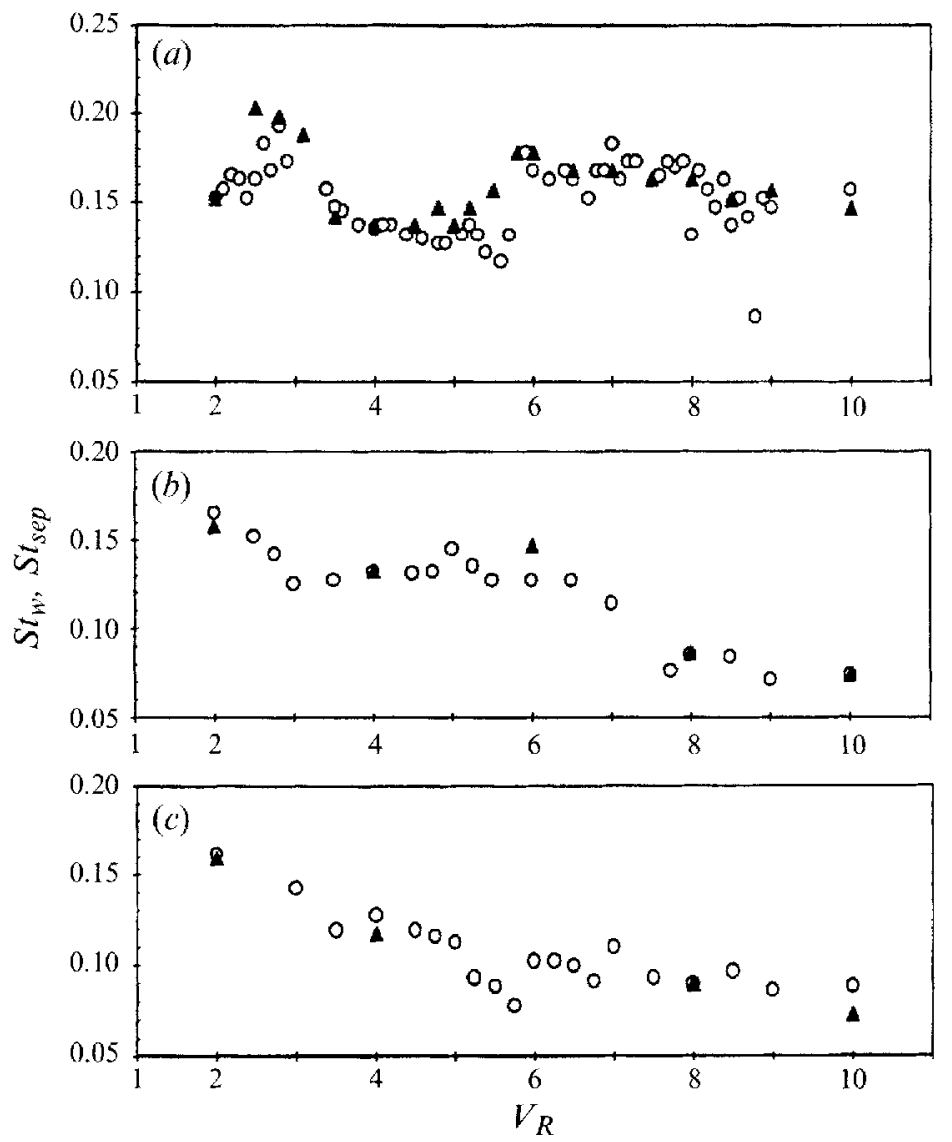

FIGURE 24. Characteristic wake Strouhal numbers $S t_{w}(O)$, and those measured near the crossflow boundary-layer separations events $S t_{\text {sep }}(\mathbf{A})$. (a) $R e_{c f}=3800 ;(b) R e_{c f}=7600 ;(c) R e_{c f}=11400$.

between the transverse-jet and cylinder flows will again be useful. Figure 25 shows visualizations in the immediate vicinity of a cylinder and a jet with $V_{R}=4$. Figures $25(a)$ and $25(b)$ show flow patterns just outside the crossflow boundary layer for the cylinder and jet, respectively. The smoke streaklines are predominantly in a plane about half a jet diameter from the wall. We re-emphasize the primary distinction between the two flows: the very near-wake region of the cylinder is open and bounded by the separating streaklines from the lateral surfaces of the cylinder while that of the jet is closed. Although the flow is unsteady and the precise flow patterns vary somewhat from image to image and from one velocity ratio to another, streaklines characteristically wrap closely around the lateral and downstream sides of the jet core.

Closure of the streakline pattern (figure $25 b$ ), approximately as in potential flow around a circular cylinder, has important implications for the boundary layer on the crossflow wall. That is, the positive pressure gradient from the diverging streamlines on the downstream sides of the jet is imposed on the wall. We believe it is this adverse pressure gradient which leads to separation of the boundary layer at the observed separation events. Although those events are unsteady, the basic effect of the pressure gradient can be illustrated qualitatively by assuming the imposed pressure field to be that of the classic steady potential flow and using Thwaites' (1949) method to calculate the response of the crossflow wall boundary layer. 
(a)

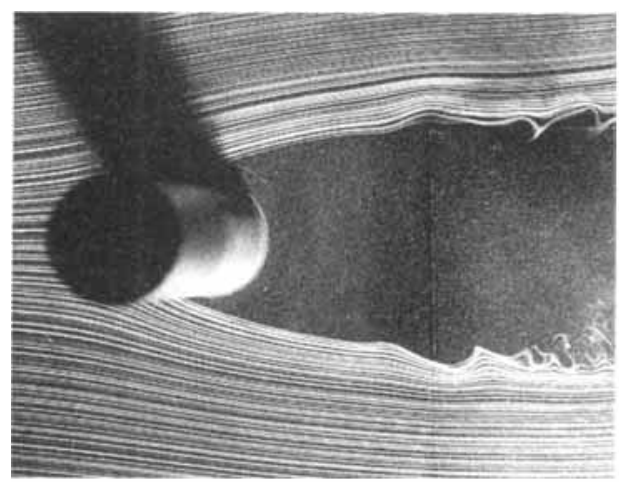

(b)

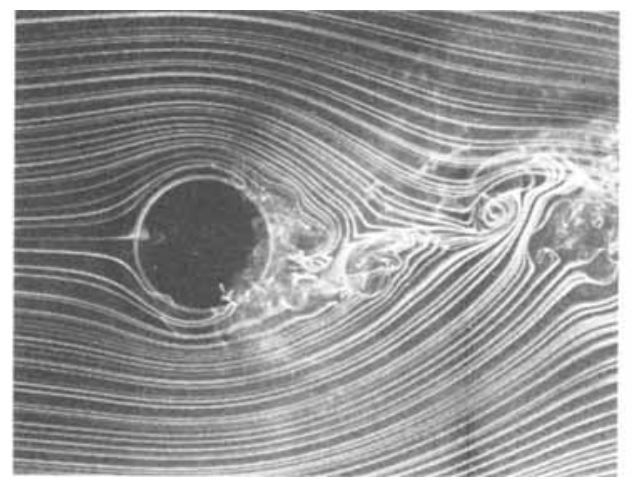

(c)

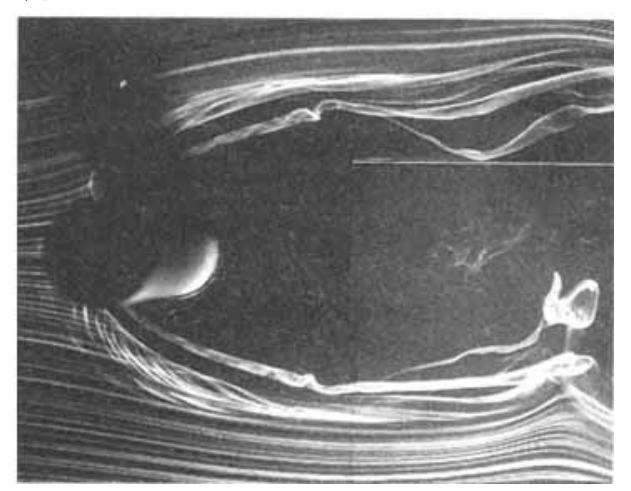

$(d)$

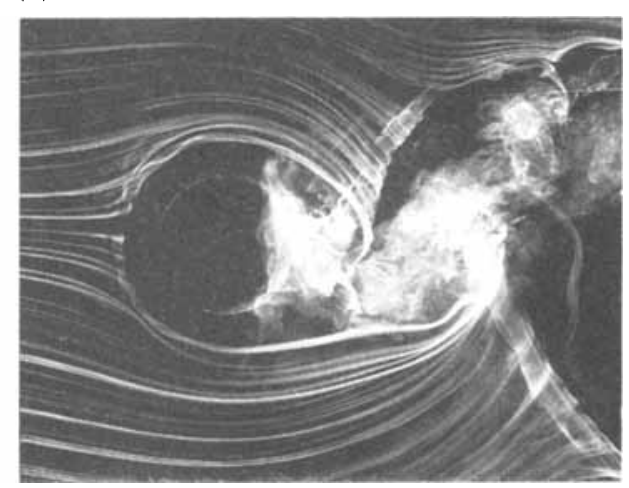

Figure 25. Near-wall flows around a cylinder and jet just above the boundary layer are compared in $(a)$ and $(b)$, respectively $\left(Z_{3 x} / D_{j}=0.5\right)$. Each flow's crossflow boundary-layer behaviour is shown in $(c)$ and $(d)$, respectively. $R e_{c f}=3800$. In $(b)$ and $(d) V_{R}=4$.

A Thwaites calculation is made along each potential-flow streamline (neglecting possible interactions between them) up to the singularity which terminates the calculation, namely zero shear stress at the wall. The loci of those singularities are shown in figure 26 . These are often called 'separation' lines but they are really only indicators of separation, which usually occurs at a somewhat different location in accord with the altered pressure distribution which accompanies separation. It is in this sense that the solid lines in figure 26 represent 'predicted' lines of 'separation'. The line upstream of the jet corresponds qualitatively to the steady separation leading to the horseshoe vortices. The line to the side and just aft of the jet corresponds to the unsteady separation event observed. (The apparent discontinuity between the two calculated separation lines is an artifact of the discrete streamlines we chose to calculate; finer spacing among them would show that the horseshoe and separation event lines join.) Compare, for instance, figures 26 and $25(d)$. The location and even the shape of the separation event of figure $25(d)$ is depicted well by this simple calculation. In figure $25(d)$, note that only the position of the roll-up of vorticity after separation is clear; the corresponding separation line must be somewhat upstream of the roll-up. An analogous comment is relevant also for the position of horseshoe vortices relative to the predicted upstream separation line.

The purpose of this calculation was not to predict the precise location of the separation events. Their locations vary with $V_{R}$, and this analysis does not take any $V_{R}$ 


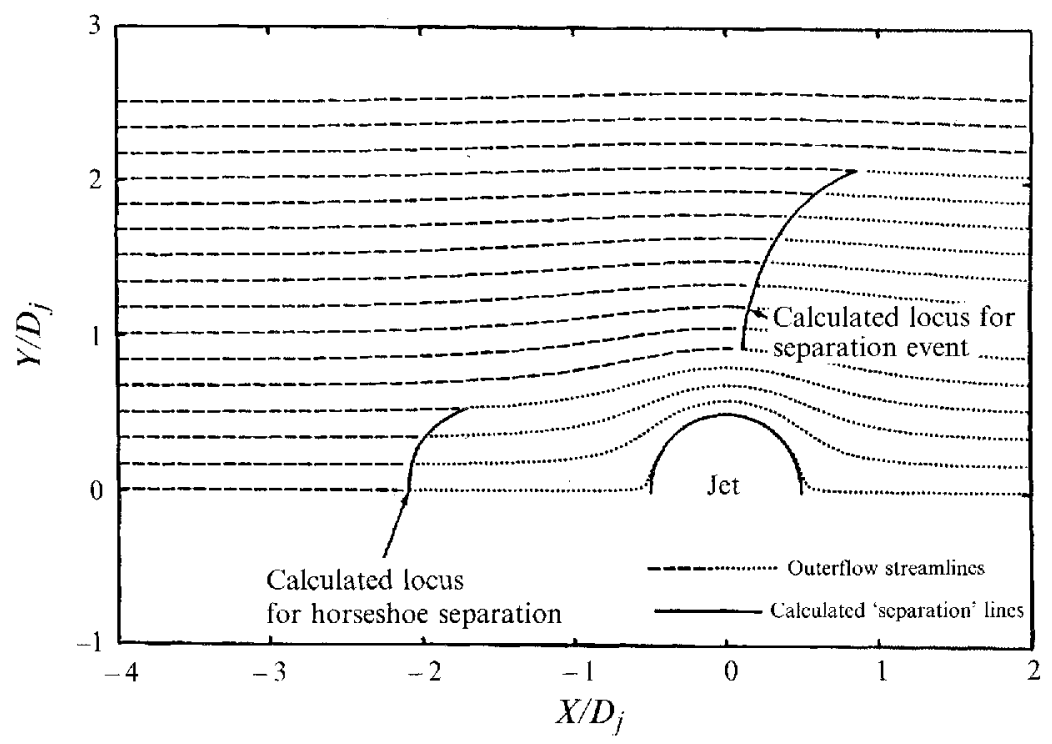

Figure 26. Predicted loci of singularities of the crossflow boundary layer near the jet orifice. A Thwaites method calculation of the crossflow boundary layer is used, and the potential flow pressure distribution of the flow about a circular section is imposed on the boundary layer. $L_{j} / D_{j}=5$.

dependence into account. Furthermore, two-dimensional laminar and steady flow is assumed while the boundary layer and outer flows here are three-dimensional and unsteady. What the analysis does is to confirm that, given the observed outer flow which closes around the jet, a separation of the crossflow boundary layer (in the area of the separation events) is expected. In the case of the solid cylinder, early separation from the surface of the cylinder itself relieves the adverse pressure gradient on both the cylinder and the crossflow wall (figures $25 a$ and $25 c$ ). The role of the initial jet diameter in setting the pressure field for the boundary-layer separation suggests its relevance for defining the wake 'Strouhal number' which was introduced in $\$ 4$. In the following subsection we consider how the separation events may be related to the formation of wake vortices.

\subsection{Wake formation mechanism}

Figure $27(a)$ shows most clearly a newly formed separation event and the accompanying near-wall structure within approximately the first 10 jet diameters of the wake. In addition to the separation event, which is marked by the arrow, several additional features are noteworthy, and the cartoon of figure $27(b)$ highlights some of these. We interpret the four relatively dense patches of smoke near the wake centreline as 'footprints' of wake vortices. Their directions of rotation are indicated by the dashed lines. Remnants of two earlier separation events, connected to the second and third wake vortices, clearly show their alternating nature. This alternating behaviour is consistently clear for wakes near $V_{R}=4$ but is not so consistent at other velocity ratios, as is evident from considering again parts $(a)$ of figures 5-9. That the wake's wall structure appears most orderly at $V_{R}=4$ is consistent with the uniqueness of $V_{R}=4$ wakes in other views of the wake and with the relatively sharp and well-defined Strouhal frequency data near $V_{R}=4$, both discussed previously.

For additional insight, a mirror was placed in the flow at $45^{\circ}$ to show, simultaneously, cross-sectional and side views of the wake. Figure 28 is an example of such a 
(a)

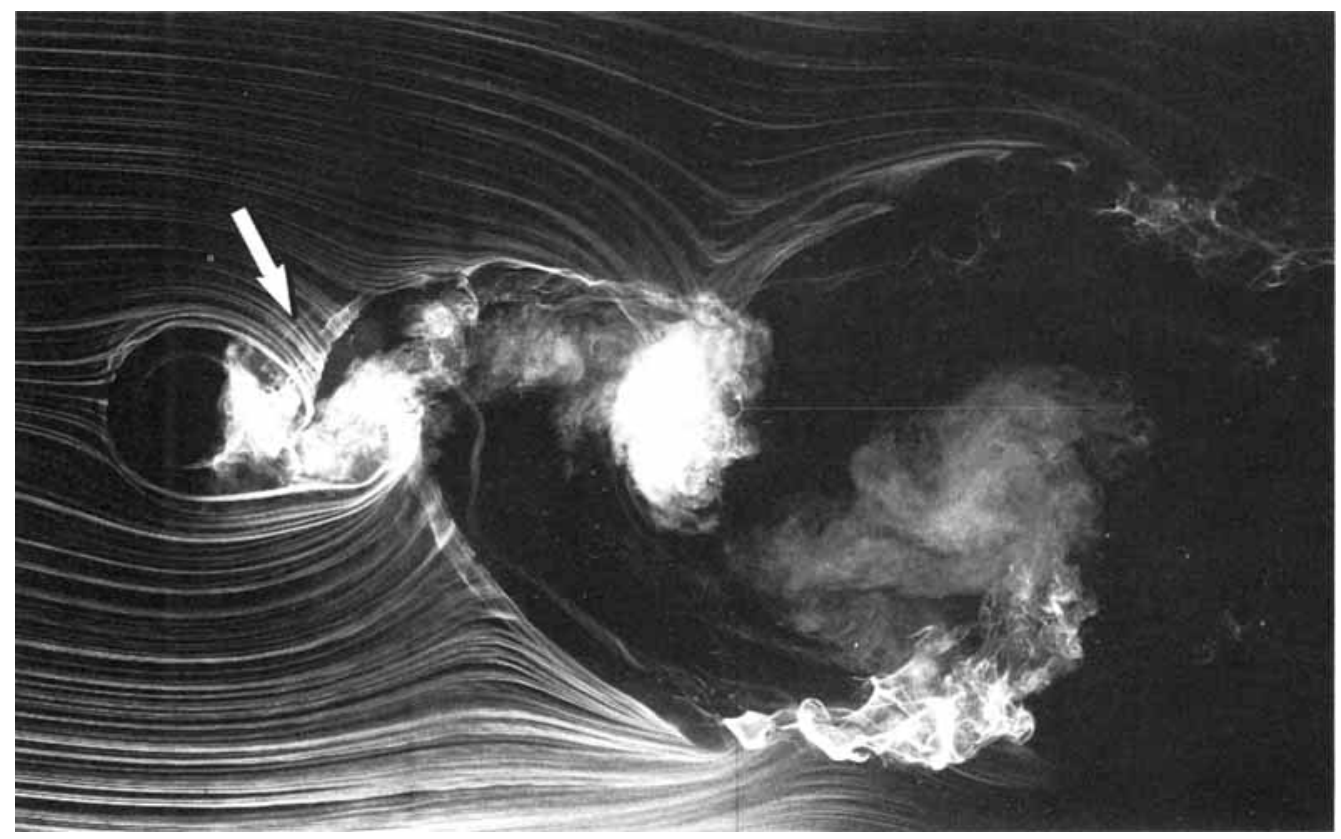

(b)

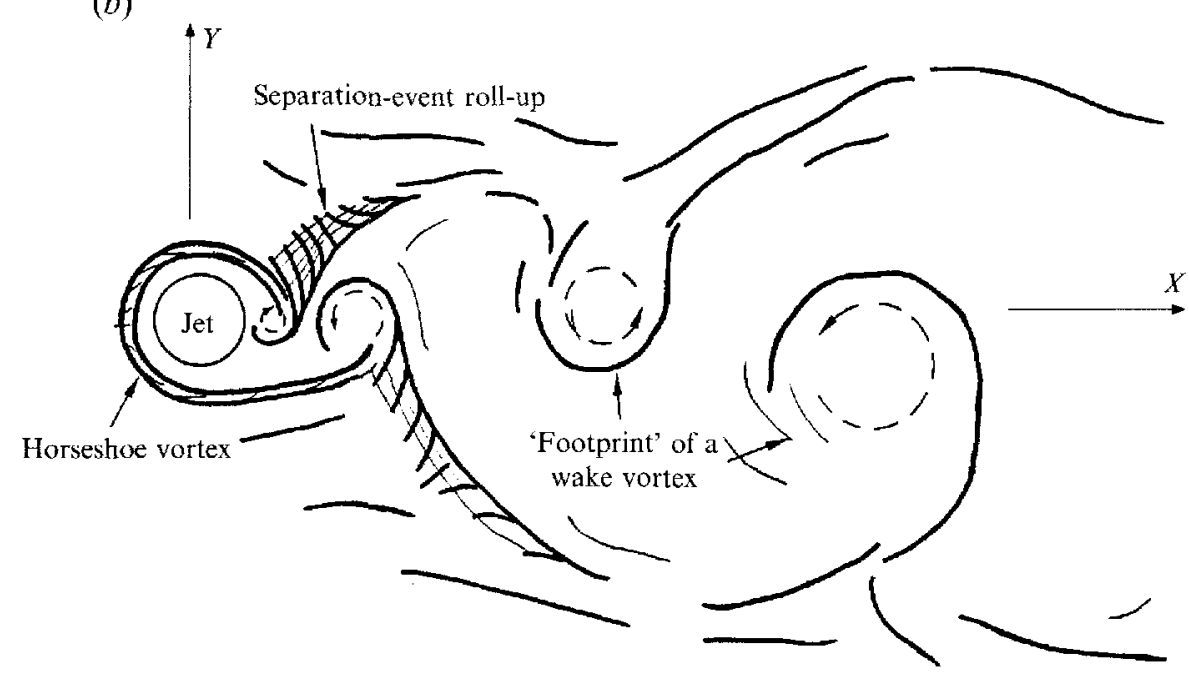

Figurf 27. Near-wall wake flow shows a newly formed separation-event roll-up and 'footprints' of several wake vortices. (a) $Z_{s w} / D_{j}=0+, V_{R}=4, R e_{i f}=3800$. (b) Cartoon highlighting structural features.

photograph and we shall refer to it to discuss the dynamics of a separation-event rollup. After separation, the vorticity from the crossflow boundary layer is 'free' to convect, stretch, turn, and diffuse, as the vorticity transport equation (3) shows. That portion of vorticity closest to the jet is entrained and convected by the jet, thus establishing a connection between the wake vortices and the jet. The vertical $(Z)$ extensions of the separation-event roll-ups into the jet are the wake vortices which are observed. The birth of a wake vortex is seen in figure 28 with the now familiar 


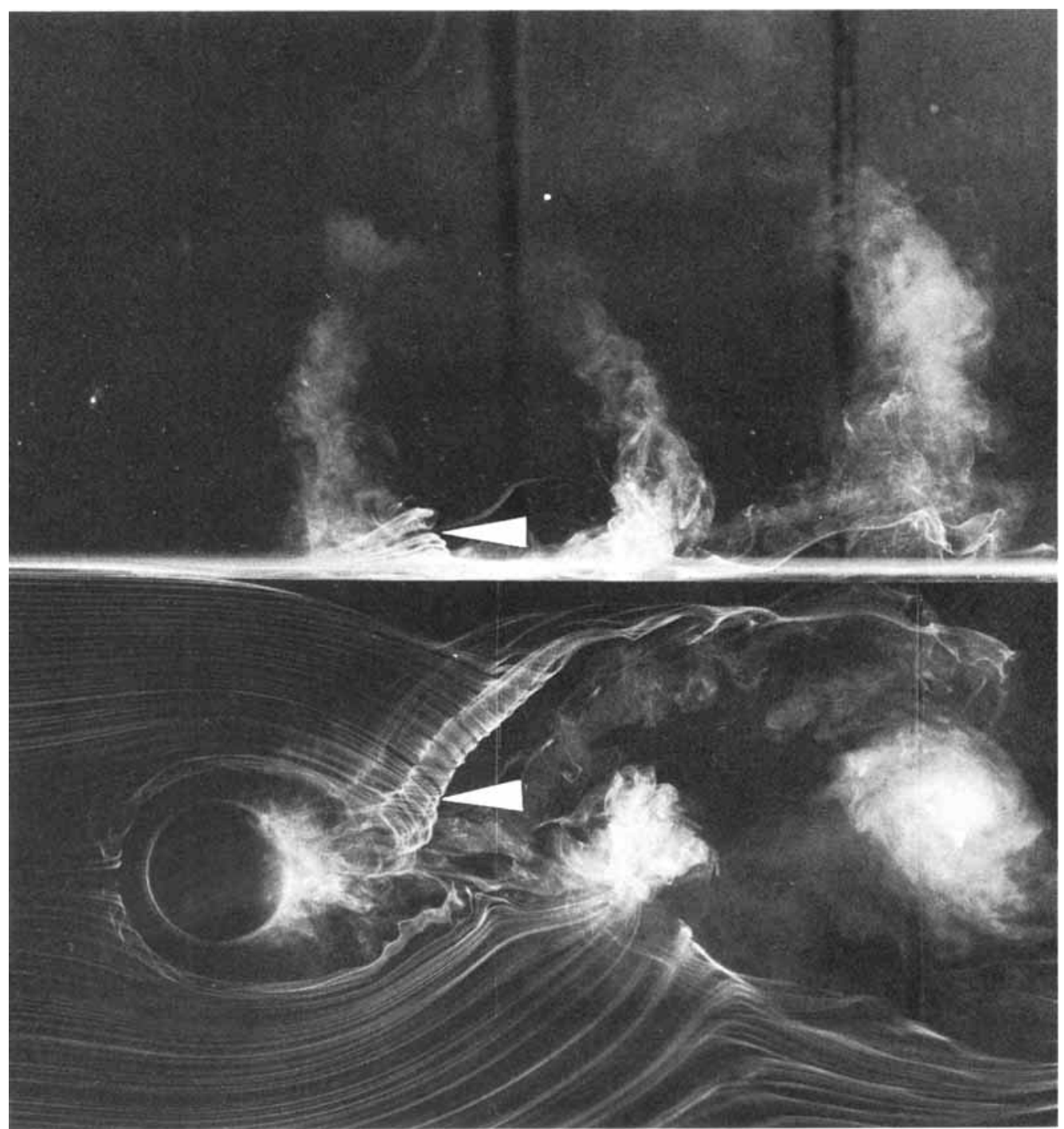

FIGURE 28. Simultaneous cross-sectional and side vicws of the wake. The arrows point to the same roll-up of boundary-layer vorticity. $V_{R}=4$ and $R e_{c f}=3800$.

separation-event roll-up on the $+Y$ side of the jet. The two arrows on the photograph indicate the separation-event roll-up and point to the same location for each view. Note, particularly on the side view, how the portion of the separation-event roll-up closest to the centreline is seen to be lifted from the wall, forming a vortical wake structure. The rotation of wake vortices whose separation events are on the $+Y$ side of the jet is clockwise, and, for those on the $-Y$ side, counterclockwise. The other 'end' of each separation event remains attached to the wall, as it must: the vortex 'sheet' of the boundary layer cannot be cut. This process of turning vorticity initially parallel to the wall is reminiscent of the 'turning-up mechanism' of Wu \& Wu (1993) discussed in $\$ 6.1$. Figure 28 also shows that the previously referred to 'footprints' of the vortices correspond well to their positions in the wake.

The significance of the separation events became apparent to us only after viewing videos of smoke-wire flow visualization. Viewing the flow from the side showed 
periodic vortical-like roll-ups just downstream of the jet and near the crossflow wall. A connection between the separation-event roll-ups and wake vortices was noticed as smoke followed a path away from the wall at the lee side of the jet and along a wake structure. The newly formed structure, extending from the crossflow wall to the jet, then was observed to convect downstream. We emphasize that axial flow along the wake vortices' cores, from the wall toward the jet, was clearly observed in the experiments.

The separation of the crossflow boundary layer and subsequent turning of vorticity into the wake structures allows the vorticity at the wall to remain tangential to the surface, as it must. As was mentioned in $\$ 6.1$, however, a wall-normal component of vorticity can exist just above the crossflow wall. In this region of the wake, $\hat{\boldsymbol{n}} \cdot\left(\boldsymbol{\nabla} \times \boldsymbol{\tau}_{\mathbf{0}}\right)$ is likely to be non-zero in the spiral flow, oriented normally to and above the crossflow wall, which is induced by each wake structure as it passes above the wall. Therefore the second vorticity source term of (4) may contribute to the vorticity in these structures. $\mathrm{Wu} \& \mathrm{Wu}$ state that this source term is responsible for the large wall-normal vorticity associated with a 'horn vortex' or a 'tornado-like vortex', descriptions applicable to the transverse-jet wake vortices. Not only do the wake structures contain vorticity already in the crossflow boundary layer, which has separated and turned, it is also possible therefore that new vorticity is continually generated at the wall as the wallnormal wake vorticity convects downstream.

Although the precise way in which the wake vortices connect with the jet is not understood, it appears that the portion of the separated boundary-layer vorticity close to the jet is entrained by it and pulled away from the wall. Relevant to this are the results of Kiya, Ohyama \& Hunt (1986). They studied the interaction of (isolated) vortex pairs and rings with a plane shear layer. For the case of strong vortex rings injected into a plane shear layer at some relative angle, they found that the vortex rings entrained and carried with them the shear-layer vortices which were in their path. The relevant analogy here is to consider the succession of (distorted) vortex rings of the bending jet as entraining and carrying portions of the separation-event roll-ups with them. Figure 29 shows a situation where this appears to be the case. In this photograph three wake vortices appear to be attached to the structure of the trailing edge of the bending jet. In the experiments of Kiya $e t$ al. the entrainment property of the vortex rings is instrumental in pulling planar shear-layer vorticity along with them. Analogously, in our experiments, it is the jet entrainment which pulls away separated vorticity from the crossflow wall.

With this understanding, we can comment on wakes which form when jets issue from pipes protruding into the crossflow. This problem was investigated by Moussa et al. (1977) who described such wakes; their experiments show that wake Strouhal numbers of protruding transverse jets more closely match Strouhal numbers of solid cylinder wakes than do those of flush-mounted jets. It may be that the natural vortex shedding from the circular cylinder (the pipe protruding into the crossflow) controls and dominates the structure in the wake. It is in fact consistent with the present results to speculate that vortices in protruding jet wakes are extensions of wake vortices from the pipe, just as the vortices in the wake of a flush-mounted jet are extensions of vorticity from the crossflow boundary layer. The entrainment by the deflecting jet stretches the pipe-cylinder's shed vortices into the wake of the jet.

We believe that the proximity of the deflecting jet to the crossflow wall is an important factor in the wake dynamics. The wake formation model described above requires the jet to entrain the closest portion of the separation-event structure away from the wall. As the vorticity from the crossflow wall is entrained by and convected 


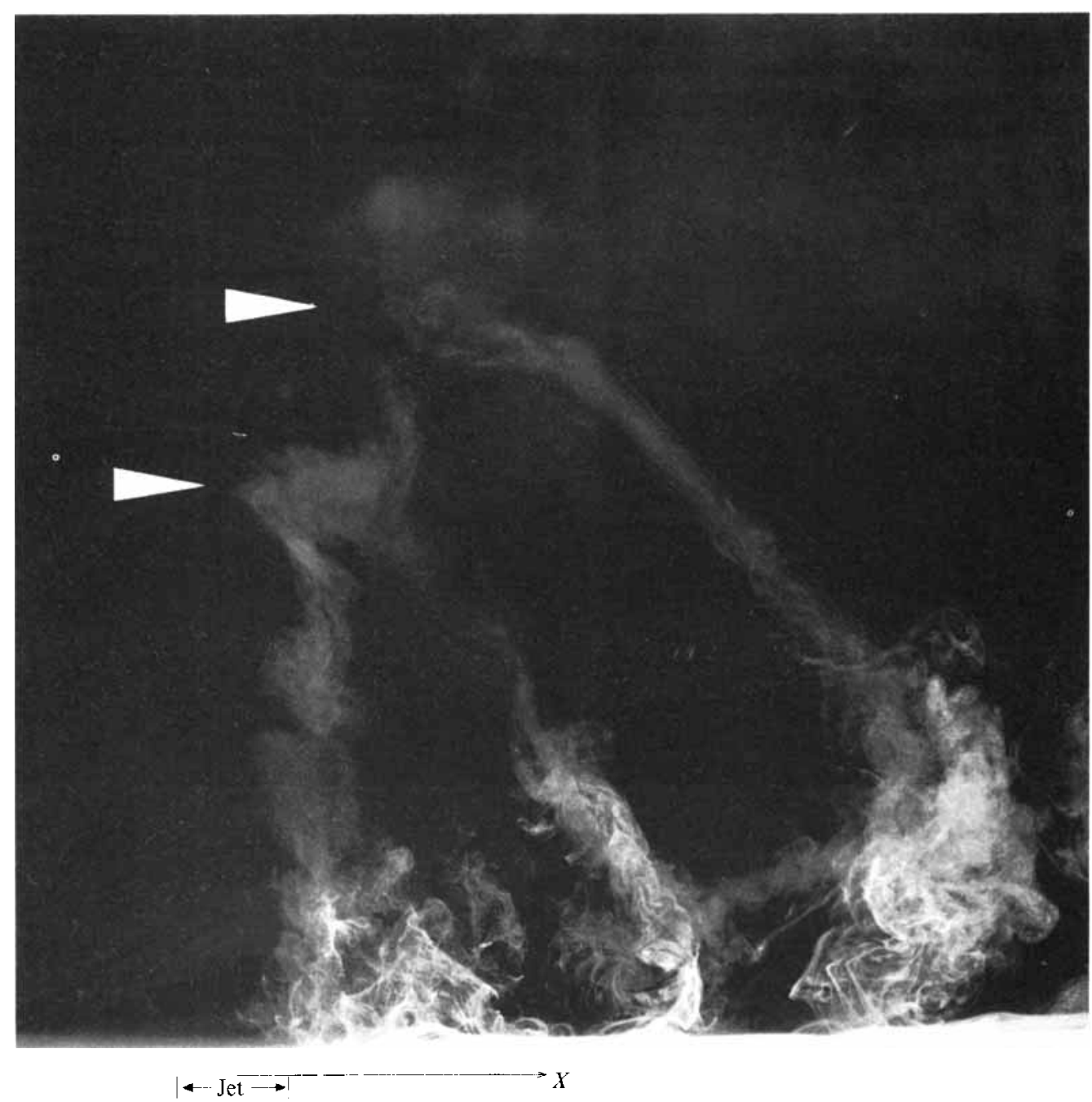

FIgURE 29. Attachment of two wake vortices to the trailing edge of the jet. $Z_{\mathrm{sx}} / D_{j}=0+$, $V_{R}=5.8$, and $R e_{c r}=3800$.

with the jet a connection between the wake vortices and the jet is established. The other end of the wake structure blends with the vorticity attached in the crossflow boundary layer. As the structures convect downstream, the wake's span increases because the jet moves further into the stream and away from the wall. We conjecture that, at some relatively large velocity ratio, the ability of the jet to entrain and affect the crossflow fluid which has separated diminishes as the jet is further removed from the crossflow wall.

Consider the sketches in figure 30, which show outlines of jet trajectories at three values of velocity ratio as determined from the smoke visualizations. These three velocity ratios are meant to represent three different regimes of the wake. The arrows in the sketches indicate an idealized (and unconfirmed) entrainment flow pattern at or near the $Y=0$ plane. At velocity ratios near 2 , the jet remains very close to the crossflow wall (figure $30 \mathrm{a}$ ). Even though its entrainment at the wall is felt very strongly, the close proximity of the jet to the wall makes it difficult to distinguish between jet, boundary-layer, and wake fluid. In a sense, the jet is too close to produce well-defined 
(a)

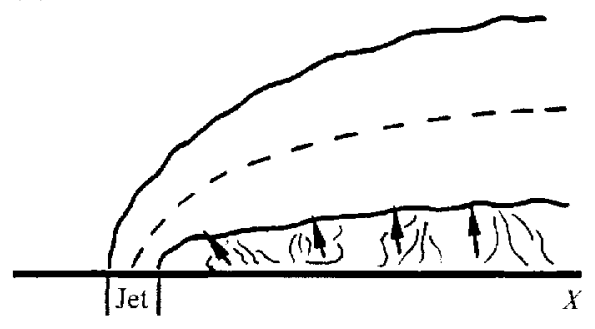

(b)

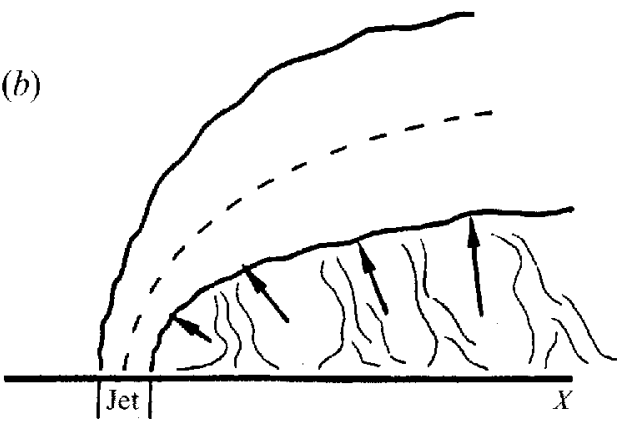

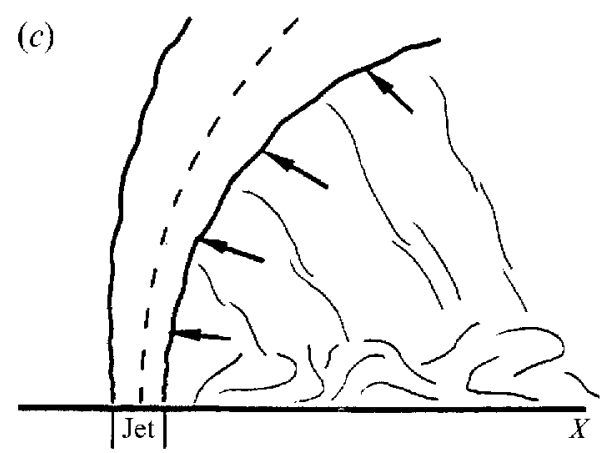

FIGURE 30. Trajectories of jets and their entrainment patterns at different velocity ratios may explain the observed dependence of wake structure on velocity ratio. (a) near $V_{R}=2 ;(b)$ near $V_{R}=4$; (c) near $V_{n}=8$.

wake structures, and it is not cleanly separated from the wall. Some stretching of the wake structures is required to define them well. Near $V_{R}=4$ (figure $30 \mathrm{~b}$ ) the jet is now far enough from the wall to induce significant turning of the separated boundary-layer vorticity, but it is close enough to still strongly and efficiently pull the separated fluid away from the crossflow wall. This moderate distance between the deflected jet and the wall allows some stretching of the wake vortices as they form, thus defining them even better. At higher velocity ratios, close to 8 (figure $30 c$ ), the crossflow boundary layer, although separated, is not easily nor efficiently entrained by the jet, for the distance of the jet from the near-wake wall region quickly becomes large. This is quite evident in the flow visualizations of the wakes at large jet velocity ratios. The side views of figure $13(d, e)$ show that the majority of smoke (initially marking the boundary-layer fluid) remains relatively close to the wall, and only thin strands extend to the jet. Also contributing to the thinness of these structures is the higher degree of stretching as the jet moves further away from the wall.

The three regimes suggested in figure 30 may also help explain the wake Strouhalnumber data shown in figure 16. To summarize those data, the main characteristics are as follows: (i) near velocity ratios of 2 and especially 4 the $S t_{w}$ data are nearly independent of $R e_{c f}$ and $L_{j} / D_{j}$; (ii) spectral peaks are relatively sharper near $V_{R}=4$; (iii) spectra are least sharp near $V_{R}=3$ and 6 ; and (iv) there is significant scatter in the values above $V_{R}=6$. We conjecture that the poorly defined spectra near $V_{R}=3$ mark the transition between the regimes shown in figures $30(a)$ and $30(b)$, i.e. the $V_{R}=2$ regime where the jet is not pulling much boundary-layer fluid from the wall and the $V_{R}=4$ regime where the jet is efficiently entraining portions of the boundary-layer fluid. Furthermore, the poor spectra near $V_{R}=6$ may mark a transition to velocity ratios at 
which the jet becomes too removed from the crossflow wall to efficiently form wake structures $\left(V_{R}=8\right.$ regime).

Complementary to these entrainment effects of jet proximity to the crossflow wall is the effect of the trajectory on that portion of the boundary layer entrained by the jet. At the higher velocity ratios, where the jet is more nearly vertical, the entrained fluid from a separation event follows a trajectory which first takes it nearly vertical, relatively far from the crossflow wall. In such a situation, it is more likely that boundary-layer/wake structure is entangled with the jet. Conversely, for velocity ratios near 4 , the entrained portion is quickly turned in the crossflow direction. The effect of this on the coherence of the wake is two-fold. First, the geometry of the situation allows the wake structures to remain more independent of the jet structure near $V_{R}=4$. Second, the closer proximity of the jet allows continual entrainment of boundary-layer fluid along the developing wake vortices.

The separation events provide a mechanism for vortical fluid to enter the wake but probably represent only the inception of the wake structures. Vorticity near the wall can be continuously fed into the wake structures as they convect downstream. This is generally what is observed. Videos show that even after the formation of a wake vortex, some spanwise or axial flow along it from the crossflow wall to the jet is seen, at least within the near field. Additionally, photographs where $Z_{s w} / D_{j}=0+$ show that the width of the disturbed flow at the crossflow wall grows with downstream distance. This is evident in figures 5-9 (parts $a$ ), and such growth suggests that crossflow boundarylayer fluid is continually entrained into the wake.

The evolution of the transverse-jet wake beyond the near field and its far-field stability is still very much an open question. Whether wake frequencies remain the same, i.e. whether vortex merging occurs, and whether these near-field structures persist over several hundred diameters and are the same ones that Kuzo \& Roshko (1984) observed, are open questions. If the vortices observed by Kuzo \& Roshko in the far field have convected from the near field, their lifespan is significantly longer than that of Kármán vortices. Possibly the continuous stretching of the jet wake and feeding by the crossflow boundary layer is instrumental.

\section{Concluding summary and discussion}

The principal finding is that the vortical structures in the wake of a jet in crossflow are not shed from the jet but are formed from vorticity which originates in the boundary layer of the crossflow wall. The mechanism by which the transverse-jet wake forms is different and distinct from the shedding of vorticity from solid cylinders. The formation process begins just downstream and to either side of the jet, with separation of the crossflow-wall boundary layer in the adverse pressure gradient which is imposed on it by the external flow, because the latter does not separate from the jet but closes around it. The 'separation event' on the crossflow wall occurs alternately on each side of the jet and results in eruptions (more or less periodically) of boundary-layer fluid and vorticity. The tornado-like vortex which is formed has its base in the boundary layer while its other end is entrained by the jet. As the vortical structure convects downstream it stretches in the widening gap between the jet and the wall; boundarylayer fluid and vorticity continue to be swept into its base.

This finding, that the wake vorticity comes from the crossflow boundary layer, emphasizes other differences between flows past transverse jets and flows past solid bluff bodies. An important difference is the absence of vorticity production at the jet-crossflow interface (when the jet and crossflow have the same density, as they do 
in our experiments). An interesting question is how this may be related to the absence of separation and vortex shedding from the jet. Another difference is that there is no 'drag' force like that which acts on a bluff body and is transmitted to the supporting wall. Instead, there is exchange of momentum between the jet and the crossflow (Coehlo \& Hunt 1989). The downstream momentum which the jet acquires must be at the expense of the crossflow and may account for some of the momentum deficit in the wake, but some of the deficit may also be introduced by the wall boundary-layer fluid that is carried into the wake in the vortical eruptions. The momentum exchange is accomplished by jet-crossflow mixing, which is dissipative. Some of this dissipation may account for part of the total pressure loss in the wake but there will also be a contribution from the dissipated wall boundary-layer fluid which enters the wake. Thus the correspondence between wake profiles of momentum deficit and of total pressure loss is not as clear as it is for solid-body wakes, and we have not sorted it out with our limited wake measurements in this complex three-dimensional flow.

The wake structure is most orderly and the Strouhal frequency scatter is smallest for jet velocity ratios near the value 4 . For those velocity ratios the eruption frequency and the corresponding Strouhal frequency of vortex passage in the wake has the value 0.13. We have argued that for this velocity ratio the jet trajectory is most favourable for formation of orderly wake structure but other factors may also have an effect. In particular, there may be effects on the wake from the other jet structures. For example, the horseshoe vortices may interact with the separation events, where the vortices originate. Interaction between the ring vortices in the jet shear layer and the wake vortices is a possibility which seems even more important and is suggested in some of the photographs. The relation between the jet shear-layer frequencies and the separation-event frequencies may determine whether wake frequencies are well defined (e.g. at velocity ratios near 4) or irregular. Finally, the evolving vortex pair, to which the wake vortices are attached, also may have a role, especially in the far field, where the mean vortex-pair structure itself contains large unsteady components that may affect or be affected by the wake vortices. The jet and its wake form an interactive system which may affect the jet itself in ways not previously considered, and the transverse jet is perhaps not as 'free' as other free shear flows.

This work benefited from earlier, exploratory experiments by D. Kuzo; from discussions with him, J. E. Broadwell and H. Hornung; and from the collaborative experiment in soap-film flow with M. Gharib. It was supported by the Office of Naval Research under contract N00014-85-K-0646 and grant N00014-89-J-1991.

\section{REFERENCES}

Becker, H. A. \& Massaro, T. A. 1968 Vortex evolution in a round jet. J. Fluid Mech. 31, 435-448.

Broadwell, J. E. \& Breidenthal, R. E. 1984 Structure and mixing of a transverse jet in incompressible flow. I. Fluid Wech. 148, 405-412.

Chaissang, P., George, J., Claria, A. \& Sananes, F. 1974 Physical characteristics of subsonic jets in a cross stream. J. Fluid Mech. 62, 41-64.

Cimbala, J. M. 1984 Large structure in the far wakes of two-dimensional bluff bodies. PhD thesis, California Institute of Technology.

Coento, S. L. V. \& Hunr, J. C. R. 1989 The dynamics of the near field of strong jets in crossflows. J. Fluid Mech. 200, 95-120.

CORKE, T., Koga, D., DruBka, R. \& NAGIB, H. 1977 A new techniquc for introducing controlled sheets of streaklines in wind tunnels. IEEE Publication 77-CH1251-8 AES. 
Couder, V. \& Basdevant, C. 1986 Experimental and numerical study of vortex couples in twodimensional flows. J. Fluid Mech. 173, 225-251.

Fearn, R. \& Weston, R.P. 1974 Vorticity associated with a jet in a cross flow. AIAA J. 12, $1666-1671$.

Freymuth, P. 1986 On transition in a separated laminar boundary layer. J. Fluid Mech. 25, 683-704.

FrIC, T. F. 1990 Structure in the near field of the transverse jet. PhD thesis, California Institute of Technology.

Fric, T. F. \& Gharib, M. 1990 Wake formation of the transverse jet. Bull. Am. Phys. Soc. 35, 2338.

Fric, T. F. \& Roshro, A. 1988 Views of the transverse jet near field. Phys. Fluids 31, 2390.

Fric, T. F. \& Roshko, A. 1991 Structure in the near field of the transverse jet. In Turbulent Shear Flows 7 (ed. F. Durst et al.). Springer.

Gharib, M. \& Derango, P. 1989 A liquid film (soap film) tunnel to study two-dimensional laminar and turbulent shear flows. Physics D37, 406-416.

Gutmark, E. \& Ho, C. M. 1983 Preferred modes and the spreading rates of jets. Phys. Fluids 26, 2932-2938.

JACOBS, J. W. 1992 Shock-induced mixing of a light-gas cylinder. J. Fluid Mech. 234, 629649.

Kamotani, Y. \& Greber, I. 1972 Experiments on a turbulent jet in a crossflow. AIAA J. 10, $1425-1429$.

Kamotani, Y. \& GRhber, I. 1974 Experiments on confined turbulent jets in crossflow. NASA CR 2392.

KefFer, J. F. \& Baines, W. D. 1963 The round turbulent jet in a cross-wind. $J$. Fluid Mech. 15, 481-496.

KiYa, M., Ohyama, M. \& HunT, J. C. R. 1986 Vortex pairs and rings interacting with shear layer vortices. J. Fluid Mech. 172, 1-15.

Kuzo, D. M. \& Roshko, A. 1984 Observations on the wake region of the transverse jet. Bull. Am. Phys. Soc. 29, 1536.

Lozano, A., Smith, S. H., Mungal, M. G. \& Hanson, R. K. 1994 Concentration measurements in a transverse jet by planar laser-induced fluorescence of acetone. AIAA J. 32, 218-221.

MCAllister, J. D. 1968 A momentum theory for the effects of cross flow on incompressible jets. $\mathrm{PhD}$ thesis, University of Tennessee.

McMahon, H. M., Hester, D. D. \& Palfrey, J. G. 1971 Vortex shedding from a turbulent jet in a cross-wind. J. Fluid Mech. 48, 73-80.

MorTon, B. R. 1984 The generation and decay of vorticity. Geophys. Astrophys. Fluid Dyn. 28, $277-308$.

Moussa, Z. M., Trisciika, J. W. \& Eskanazi, S. 1977 The near field in the mixing of a round jet with a cross-stream. J. Fluid Mech. 80, 49-80.

Pratte, B. D. \& Baines, M. 1967 Profiles of the round turbulent jet in a crossflow. J. Hydronaut. Div. ASCE 92, 53-64.

RFilLLY, R. S. 1968 Investigation of the deformation and penetration of a turbulent, subsonic jet issuing transversely into a uniform, subsonic main stream. PhD thesis, University of Maryland.

SCORer, R. S. 1958 Natural Aerodynamics. Pergamon Press.

Thwaltis, B. 1949 Approximate calculation of the laminar boundary layer. Aero. Q. 1, 245-280.

WU, J. M., VAKILI, A. D. \& YU, F. M. 1988 Investigation of the interacting flow of nonsymmetric jets in crossflow. AIAA J. 26, 940-947.

Wu, J.Z., Gu, J. W. \& WU, J. M. 1987 Steady three-dimensional fluid particle separation from arbitrary smooth surface and formation of free vortex layers. 5th AIAA Appl. Aerodyn. Conf. Monterey.

WU, J. Z. \& WU, J. M. 1993 Interaction between a solid surface and a viscous compressible llow field. J. Fluid Mech. 254, 183-211. 